\title{
Organische Spurenstoffe als Indikatoren zur \\ Charakterisierung komplexer Grundwassersysteme
}

\section{Ansätze am Beispiel eines urban geprägten \\ Karstgrundwasserleiters}

Dissertation

zur Erlangung des mathematisch-naturwissenschaftlichen Doktorgrades

"Doctor rerum naturalium"

der Georg-August-Universität Göttingen

im Promotionsprogramm Geowissenschaften

der Georg-August University School of Science (GAUSS)

vorgelegt von Roland Reh

aus Göttingen

Göttingen 2014 
Betreuungsausschuss:

Referent:

Korreferent:

Weitere Mitglieder der Prüfungskommission:
PD Dr. Tobias Licha,

Geowissenschaftliches Zentrum

Angewandte Geologie

Georg-August-Universität Göttingen

Prof. Dr. Martin Sauter

Geowissenschaftliches Zentrum

Angewandte Geologie

Georg-August-Universität Göttingen

PD Dr. Tobias Licha

Geowissenschaftliches Zentrum

Angewandte Geologie

Georg-August-Universität Göttingen

Prof. Dr. Martin Sauter,

Geowissenschaftliches Zentrum

Angewandte Geologie

Georg-August-Universität Göttingen

Prof. Dr. Hans Ruppert,

Geowissenschaftliches Zentrum

Georg-August-Universität Göttingen

PD Dr. Chicgoua Noubactep,

Geowissenschaftliches Zentrum

Georg-August-Universität Göttingen

PD Dr. Ekkehard Holzbecher

Geowissenschaftliches Zentrum

Georg-August-Universität Göttingen

Dr. Tobias Geyer,

Landesamt für Geologie,

Rohstoffe und Bergbau (LGRB)

im Regierungspräsidium Freiburg

Tag der mündlichen Prüfung: $\quad$ 24.10.2014 


\section{Kurzfassung}

In komplexen Grundwasserleitern lassen sich grundlegende Informationen zur Abgrenzung von Quelleinzugsgebieten, zur Ableitung von Grundwasserfließrichtungen oder zur Bewertung der Vulnerabilität nicht allein über konventionelle hydrogeologische Methoden (beispielsweise Grundwassergleichenpläne, Quellschüttungsmessungen, Pumpversuche) gewinnen, so dass vielfach ergänzend hydrochemische Methoden eingesetzt werden. Zu den gängigen hydrochemischen Methoden gehören Auswertungen der Hauptionenverhältnisse, stabiler und radioaktiver Isotope, von Umwelttracern und Metallen der Seltenen Erden einschließlich des Yttriums sowie der aktive Einsatz künstlicher Markierungsstoffe. Die Anwendung gängiger hydrochemischer Methoden ist durch natürliche oder anthropogene Hintergrundkonzentrationen, die die Ausbildung eines ausreichenden Kontrasts behindern können, limitiert. Da diese Methoden meist nur an der Quelle als integrales Signal des gesamten Einzugsgebiets ansetzen, kann die Abgrenzung des Einzugsgebiets nur indirekt durch die Abschätzung von Volumina und darauf aufbauender Abschätzung der Flächengröße vorgenommen werden. Zur konkreten Lokalisierung der Einzugsgebietsgrenzen und der Ableitung von Grundwasserfließrichtungen sind also zusätzliche Informationen notwendig.

Durch die menschliche Produktion organischer Verbindungen steht ein riesiges Spektrum potentieller Indikatoren zur Verfügung, das die Anzahl der bei etablierten Methoden eingesetzten Stoffe um ein Vielfaches übertrifft. Aufgrund ihrer rein anthropogenen Herkunft haben organische Spurenstoffe keine natürlichen Hintergrundkonzentrationen und sind daher bereits auf einem sehr geringen Konzentrationsniveau nutzbar.

Im Gebiet der „Waldecker Scholle“ konnten sowohl die räumliche Verteilung organischer Spurenstoffe als auch deren saisonale Konzentrationsschwankungen in einem komplexen Grundwassersystem mit moderat verkarsteten Grundwasserleitern untersucht werden. Die auftretenden Stoffmuster reflektieren die hydrogeologischen Verhältnisse und können zur Lokalisierung von Quelleinzugsgebietsgrenzen genutzt werden. Weitere Hinweise liefern die organischen Spurenstoffe zur Identifikation von punktuellen Eintragsquellen und zur Ausbreitung der aus Punktquellen freigesetzten Stoffe im Grundwasserleiter sowie zum Attenuationspotential dieser Stoffe. Weiterhin kann die hydraulische Wirksamkeit von geringleitenden Zwischenschichten und Störungszonen mittels Identifikation einer Fließkomponente mit einer Verweilzeit von mehr als 20 Jahren abgeschätzt werden. Auch Annahmen hinsichtlich der Vulnerabilität lassen sich validieren. 
Der Vergleich mit dem hydrogeologisch gut untersuchten Einzugsgebiet der Gallusquelle, zeigt, dass Stoffgruppen wie Triazine und Triazole eine weite Verbreitung haben, andere Stoffe jedoch an bestimmte Gegebenheiten im Gebiet gebunden sind (beispielsweise Altablagerungen, Kanalsystem). Außerdem bestätigt sich in beiden Gebieten, dass das Auftreten von Stoffen sowohl von den Stoffeigenschaften als auch von den hydrogeologischen Eigenschaften abhängig ist. Das bedeutet, dass die Art der in einer Karstquelle auftretenden Stoffe selbst ohne aufwändige zeitlich hochaufgelöste Beprobung bereits Rückschlüsse auf Systemeigenschaften zulässt.

Die vorliegende Arbeit zeigt somit, dass organische Spurenstoffe bereits auf der Untersuchungsebene des konzeptionellen Modells wertvolle Indikatoren zur Charakterisierung komplexer Grundwassersysteme sein können. Die große Menge an zur Verfügung stehenden Stoffen bietet die Möglichkeit, bestimmte Stoffe auszuwählen, die die jeweiligen Systemcharakteristika widerspiegeln. Mit organischen Spurenstoffen können sowohl Grundwasserkomponenten unterschiedlicher Verweilzeiten von wenigen Tagen bis $\mathrm{zu}$ mehreren Jahrzehnten identifiziert werden als auch räumliche Zusammenhänge von wenigen Metern bis über Einzugsgebietsgrenzen hinaus untersucht werden. Die Einbeziehung organischer Spurenstoffe in die Erkundungsstrategien komplexer Grundwassersysteme hat daher ein vielversprechendes zukunftsweisendes Potential. 
Conventional hydrogeological methods (e.g. water level contour maps, hydrographs, pumping tests) can only provide limited information for the basic characterization of complex aquifers, namley catchment delineation, deriving groundwater flow directions or the assessment of vulnerability. Therefore, hydrochemical methods are additional applied in many studies. Conventional hydrochemical methods comprise analysis of the major ions, stable and reac-tive isotopes, environmental tracers, rare earth elements including Yttrium as well as the application of artificial tracer substances. However, the application of these methods is limited by geogenic or anthropogenic background concentrations which may inhibit the development of an adequate contrast. The catchments can only be indirectly delineated by these methods on the base of recharge volumes and the following estimation of the catchment size, because they rely only on the spring signal which integrates the response of the whole catchment. Consequently, for the localization of the catchment boundaries and deriving flow directions additional information are needed.

An enormous spectrum of potential indicators is available by the anthropogenic production of organic compounds, exceeding the number of compounds of the conventional methods many times over. Due to their anthropogenic origin, organic compounds have no geogenic background. Therefore, they can be even used at very low concentrations.

With the research, conducted in the study area "Waldecker Scholle“, the spatial distribution of organic micro-pollutants (OMPs) as well as the seasonal variation of their concentrations were investigated in a complex groundwater system with moderately karstified aquifers. Since, the hydrogeological properties are reflected by the patterns of seasonal and spatial distribution, OMPs can be applied for the localization of catchment boundaries. OMP point sources and their influence on the aquifer as well as the potential for attenuation of the released compounds are also indicated. Additional, the hydraulic properties of aquitards can be estimated by an identified flow component with a residence time of more than 20 years. Also, the vulnerability can be evaluated.

By comparing the findings with the well investigated Gallusquelle catchment it is demonstrated that certain compound classes, such as triazines and triazoles exhibit a broad occurrence, while the occurrence of other compounds depends on the local conditions in the study areas (e.g. old waste disposals or the sewer system). Moreover, it was found that in both study areas the occurrence of compounds depends on the chemical and physical properties of the OMPs as well as on the hydrogeological properties. Consequently, the type of 
compounds occurring at a spring allows drawing conclusions regarding the hydrogeological properties of the catchment, even without conducting sampling campaigns with a high temporal resolution.

The present dissertation, therefore, demonstrates that OMPs are valuable indicators, even on the study level of a conceptual model. The large number of available compounds offers the opportunity to select compounds that reflect the characteristics of the investigated system. With organic micro-pollutants both, water components with residence times of a few days up to several decades and spatial relationships of a few meters up to over the boundaries of a catchment area can be identified. The inclusion of organic micro-pollutants into investigation strategies of complex groundwater systems, therefore, has a promising forward-looking potential. 


\section{Vorwort}

Persönlicher Ausgangspunkt meiner Dissertation ist die sog. Waldecker-Scholle, in der ich in den vergangenen fünfzehn Jahren als Gutachter mehrere kleinere und größere Altlasten und Altlastenverdachtsflächen hinsichtlich ihres Einflusses auf das Grundwasser zu bewerten hatte. Die drei dort vorhandenen teils verkarsteten und teils hydraulisch verbundenen Grundwasserleiter in den Karbonatformationen des Zechsteins (Karbonate der Werra-, Staßfurt- und Leine-Formation) bilden einerseits ein komplexes Fließsystem und decken andererseits die lokale Trinkwasserversorgung nahezu vollständig ab.

Die qualitative und quantitative Erfassung der Fließ- und Transportprozesse in solchen verkarsteten Grundwasserleitern ist jedoch generell schwierig, da diese heterogen, anisotrop und durch das Zusammenwirken unterschiedlicher Fließkomponenten charakterisiert sind. Aufgrund des hohen materiellen Aufwands und der geringen räumlichen Repräsentativität sind einer Erkundung mittels Aufschlussbohrungen Grenzen gesetzt. Dieses gilt auch für das betrachtete Gebiet, in dem in der Vergangenheit zwar zahlreiche hydrogeologische und altlastentechnische Gutachten erstellt wurden, die jedoch auf räumlich oder zeitlich begrenzten Untersuchungen beruhen und daher die Zusammenhänge zwischen Schadstoffeinträgen und Trinkwassergewinnungsanlagen nicht zufriedenstellend klären konnten.

In der Vergangenheit durchgeführte Untersuchungen mit künstlichen Markierungsstoffen führten nicht zu einer in sich schlüssigen konzeptionellen Modellvorstellung. Die Hauptschwierigkeiten solcher Untersuchungen resultieren einerseits gerade aus dem Mangel an grundlegenden Informationen, wie die Abgrenzung von Einzugsgebieten und die Bestimmung von Fließrichtungen, die eine aufwändige Versuchsgestaltung erfordern würden und andererseits aus dem Mangel an geeigneten Eingabestellen für künstliche Markierungsversuche im überbauten Stadtgebiet. Daher entstand die Idee, zu überprüfen, ob organische Spurenstoffe, die den Vorteil bieten, dass sie durch menschliche Aktivität bereits in das Grundwassersystem eingetragen wurden, als Indikatoren zur Erstellung eines konzeptionellen Modells genutzt werden können. 


\section{Danksagung}

Mein besonderer Dank gilt Herrn Prof. Dr. Sauter, der mir das notwendige Vertrauen geschenkt dafür hat, diese Arbeit zum Großteil außerhalb der Räumlichkeiten des Geowissenschaftlichen Zentrums der Universität Göttingen anzufertigen. Ebenso danke ich ganz besonders meinen Betreuern Herrn PV Dr. Tobias Licha und Herrn Dr. Tobias Geyer, die stets Rücksicht auf diese Situation genommen haben und mit denen durch zahlreiche fruchtbare und anregende Diskussionen viele Ideen entstanden. Ihnen möchte ich auch für die sprachliche Unterstützung bei der Erstellung der englischsprachigen Artikel bedanken.

Das gesamte Projekt hätte nicht realisiert werden können, ohne die finanzielle Unterstützung des Hessischen Landesamtes für Umwelt und Geologie (HLUG) und der Energie WaldeckFrankenberg GmbH (EWF). Hiervon sind namentlich Frau Schlösser-Kluger, Frau Dr. JaegerWunderer vom HLUG sowie Herr Schaller und Herr Wilke von der EWF hervorzuheben. Weiterhin ist zahlreichen Mitarbeitern dieser beiden Institutionen, des Regierungpräsidiums Kassel sowie der Stadt Korbach für das Bereitstellen grundlegender hydrogeologischer Daten und Informationen zu danken. An dieser Stelle möchte ich auch Frau Iris König von der Stadt Korbach, die in der Startphase des Projekts wichtige Kontakte hergestellt hat, in den Dank einbeziehen.

Diese Arbeit hätte keine Grundlage ohne die Analysen der organischen Spurenstoffe. Deswegen möchte ich mich bei Dr. Karsten Nödler für die sorgfältigen Messungen sowie dem Laborteam mit Mechthild Rittmeier und Anke von Gaza für ihre große Flexibilität sowie die ständige Hilfsbereitschaft bedanken.

Ganz besonderer Dank gilt meiner Frau Andrea und meinen Söhnen Jonas und Simon, die durch ihre Geduld und ihre Bereitschaft, mir die nötigen Freiräume zu geben, eine Grundvoraussetzung für das Zustandekommen dieser Arbeit geschaffen haben. In gleiche Richtung geht mein Dank an die Geschäftsführerkollegen der Geonik GmbH.

Nicht vergessen in dieser Danksagung möchte ich die Unterstützung meiner DoktorandenKollegen, Eltern und Freunde. 


\section{Inhaltsverzeichnis}

1 Einleitung.

1.1 Probleme und Fragestellungen der hydrogeologischen Charakterisierung von komplexen Grundwasserleiter- und Karstsystemen. 1

1.2 Zielsetzung dieser Arbeit. 3

1.3 Geologische und hydrogeologische Charakteristika des Untersuchungsgebiets .......... 4

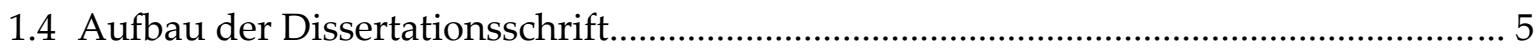

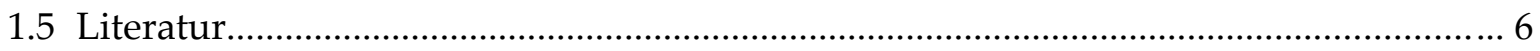

2 Charakterisierung großräumiger komplexer Grundwassersysteme und

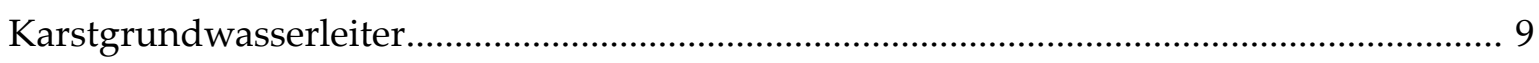

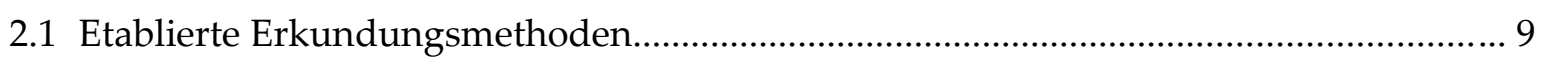

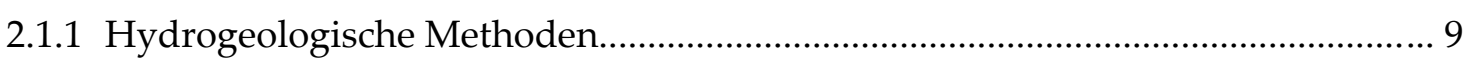

2.1.2 Nutzung hydrochemischer Daten zur Charakterisierung von

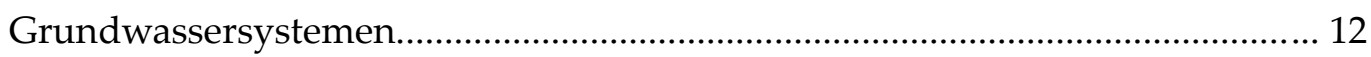

2.1.3 Zusammenfassung der Einschränkungen etablierter hydrochemischer Methoden.

2.2 Nutzung organischer Spurenstoffe zur Charakterisierung von Grundwassersystemen.

2.2.1 Definition und Eigenschaften organischer Spurenstoffe. 25

2.2.2 Bisheriger Stand zur Nutzung organischer Spurenstoffe als Transportindikatoren in aquatischen Systemen.................................................. 26

2.3 Literatur. 28

3 Occurrence and spatial distribution of organic micro-pollutants in a complex hydrogeological karst system during low flow and high flow periods, results of a twoyear study

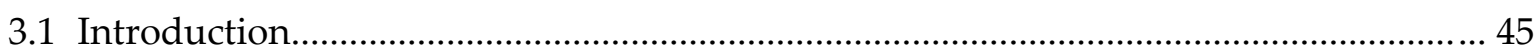

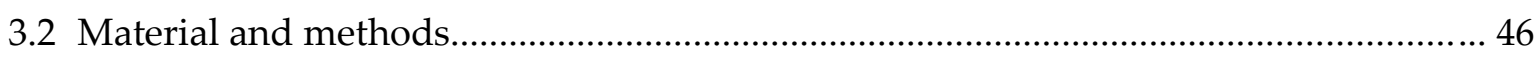

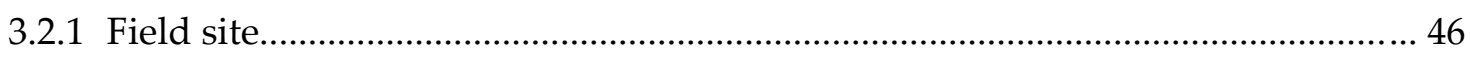

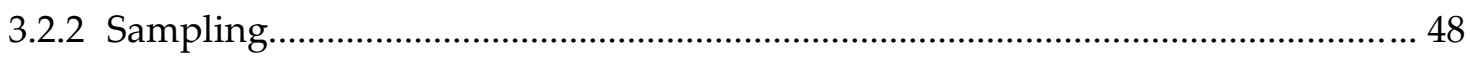

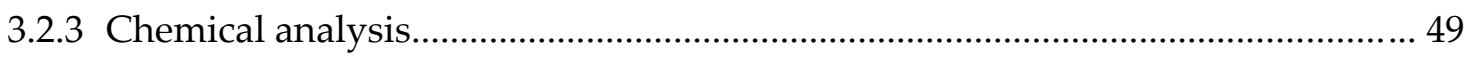

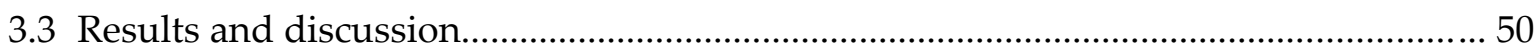

3.3.1 Detection frequency of OMPs in groundwater................................................. 50

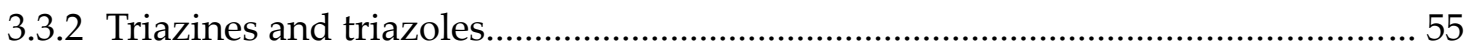

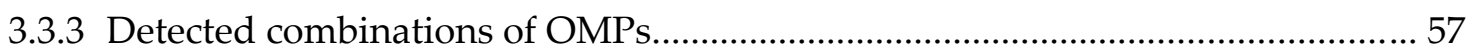

3.3.4 Environmental implication of detected OMPs.................................................... 58 


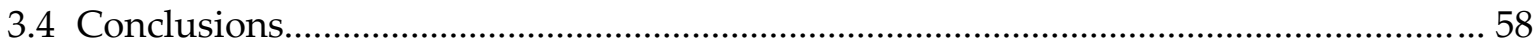

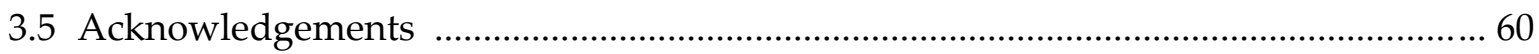

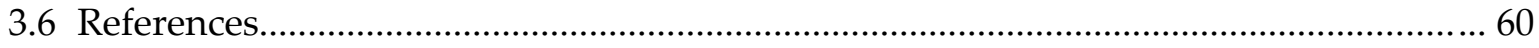

4 Evaluation and application of organic micro-pollutants (OMPs) as indicators in karst

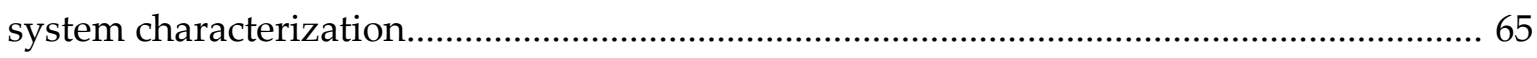

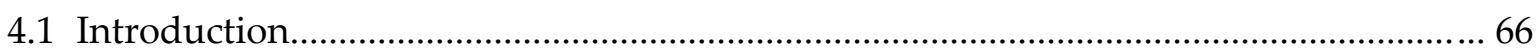

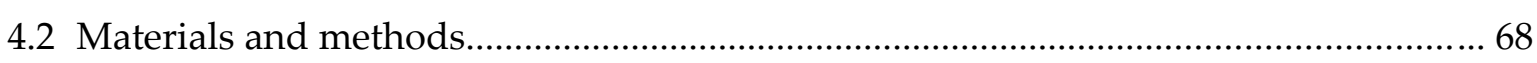

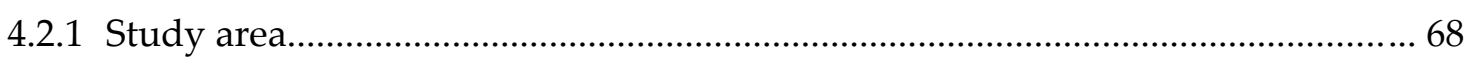

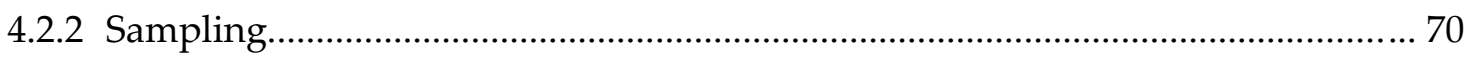

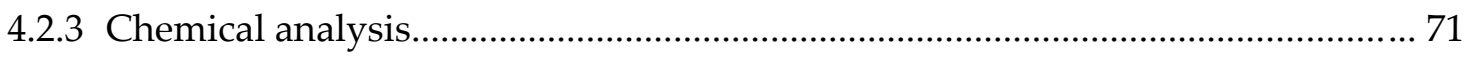

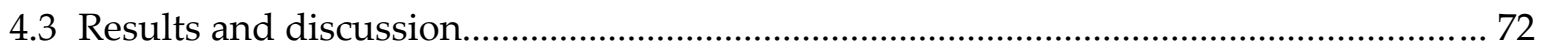

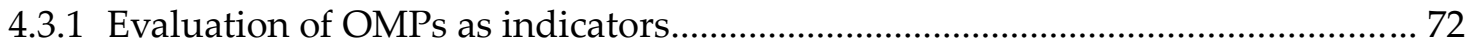

4.3.2 Application of OMP as indicators in a complex karst system in support of the development of a conceptual flow model............................................................... 77

4.3.3 Detection and characterization of contaminant sources...................................... 82

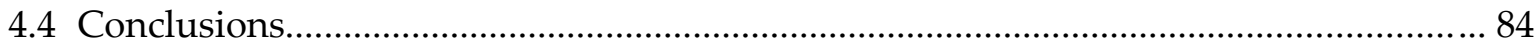

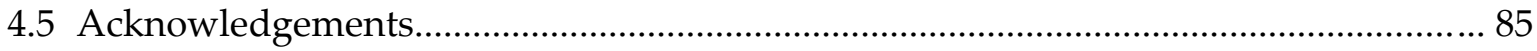

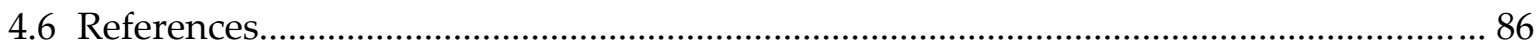

5 Charakterisierung zweier Karstsysteme mit Hilfe organischer Spurenstoffe..................... 93

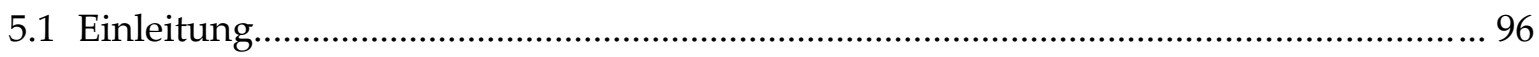

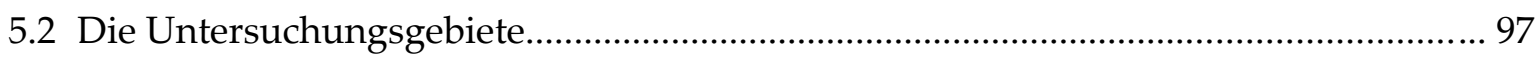

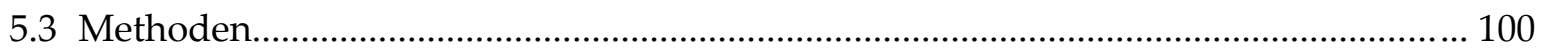

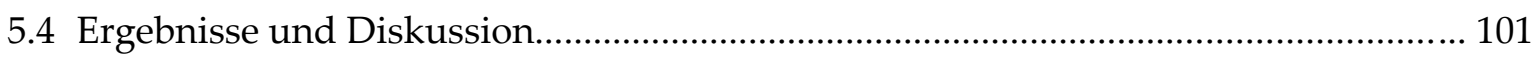

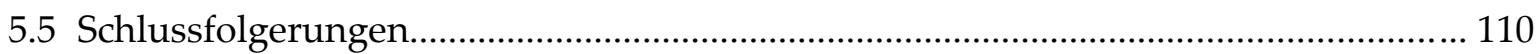

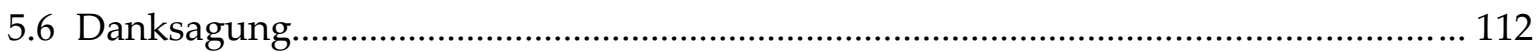

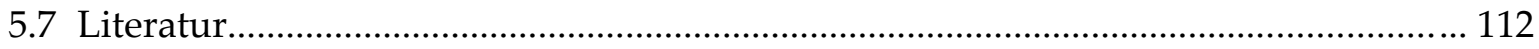

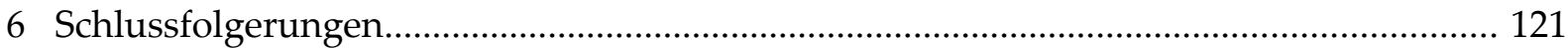

6.1 Möglichkeiten der Nutzung organischer Spurenstoffe zur Charakterisierung

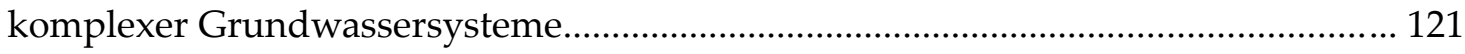

6.2 Organische Spurenstoffe zur Charakterisierung von Grundwasserleitern im Vergleich

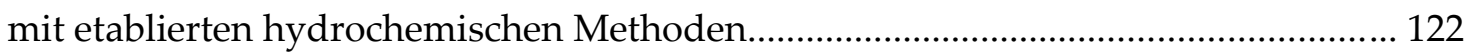

6.3 Offene Fragen und Perspektiven der Nutzung organischer Spurenstoffe zur Charakterisierung komplexer Grundwasserleiter.................................................... 126

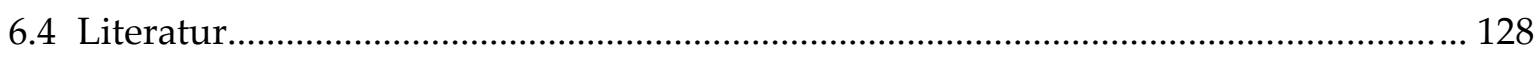




\section{Abbildungsverzeichnis}

Abb. 1.1: Skalen von Gebirgsdurchlässigkeiten (nach Worthington 2003) und Verweilzeiten (nach Worthington 2007) in Karstsystemen

Abb. 1.2: Lage der Waldecker Scholle, Grundwasserbelastungen mit Nitrat und LHKW, Verteilung von Altflächen, Rechts- und Hochwerte als Gauß-Krüger-Koordinaten. 5

Fig. 3.1: Study area with characterization of land uses, geological structures and estimated groundwater flow directions, water divide according to

Hölting \& Matthess (1963).

Fig. 3.2: Number of different OMPs per sampling point as median of all sampling campaigns with values of standard deviation

Fig. 3.3: Ternary diagram of triazine mass fractions, data from sampling campaigns March 2011 and September 2011, same results are obtained in March and August 2010..... 56

Fig. 3.4: Main sources of OMPs and principles of transport. 59

Fig. 4.1: Characteristics of the study area (Reh et al. 2013), geological setting and stratigraphy of the Zechstein sequences (z1-zFb).

Fig. 4.2: Spatial distribution of the number of detected OMPs per sampling point, assigned to different types of applications, size of the pie charts depends on the detected number of OMPs.

Fig. 4.3: Seasonal deviation of tolyltriazole and DEA concentrations in different types of sampling points, areas and aquifers, in relation to the discharge of the river in the North

Fig. 4.4: Spatial distribution of triazine concentrations (atrazine, DEA, DIA) and BT concentrations in August 2010, catchment delineation of the spring areas and correlation with base contour lines of the Upper Aquifer.

Fig. 4.5: Composition of major ions as Piper plot demonstrated with the samples of August 2010, similar results are obtained with the data from the other sampling campaigns

Fig. 4.6: Spatial extension of OMP concentrations originating from a disused waste disposal. 
Abb. 5.1: a) Geologischer Profilschnitt der Waldecker Scholle nach Käding (2005) und Kulick (1997) b) Übersichtskarte der Waldecker Scholle mit Lage der Probennahmestellen c) Übersichtskarte des Einzugsgebiets der Gallusquelle verändert nach Geyer et al. (2007) d) Geologischer Profilschnitt des Einzugsgebiets der Gallusquelle nach Sauter (1992)

Abb. 5.2: Auftreten und Konzentrationen von Makrolid-Antibiotika und dem Antiallergikum Loratadin in Grundwasserproben der Waldecker Scholle mit Bezug zum Abfluss der Twiste. 106

Abb. 5.3: Zeitliche Verteilung der Konzentrationen von Isoproturon, Iohexol und Carbamazepin an der Gallusquelle. Die unterbrochenen senkrechten Linien stellen dokumentierte Überlaufereignisse des Regenrückhaltebeckens dar.

(rote Symbole: Messwert < NG; blaue Symbole: NG < Messwert < BG;

schwarze Symbole: Messwert > BG). 107 


\section{Tabellenverzeichnis}

Tab. 3.1: Stratigraphic table of the local Zechstein formations based on Menning \& Hendrich (2002) with identification of the aquifers and allocation of sampling points. 48

Tab. 3.2: Electrospray (ESI)-MS-MS-parameters of the analytes additional to Nödler et al. (2010) 50

Tab. 3.3: Detection frequency of OMPs relating to the number of groundwater samples with minimum- (min.), median- and maximum- (max.) concentrations of all groundwater samples......

Tab. 3.4: Number of DDD in the investigation area in 2010, value of DDD and estimated potential input mass.

Tab. 4.1: Most frequently detected OMPs, allocated to the water body of detection. 76

Tab. 5.1 Organische Spurenstoffe mit Anwendungsgebieten und Bestimmungsgrenzen, aufgeführt sind Stoffe mit Detektionshäufigkeiten $\geq 10 \%$ quantifizierbarer Konzentrationen (absteigende Sortierung) in den Grundwasserproben beider Untersuchungsgebieten (Angaben zur Waldecker Scholle aus Reh et al. (2013)) 101

Tab. 5.2 Vergleich der Min.- Median- und Max.-Konzentrationen der Befunde >BG mit Angabe der Detektionshäufigkeit, absteigend sortiert nach Detektionshäufigkeit. 103

Tab. 5.3 Absatzmengen der untersuchten Pestizide

als Pflanzenschutzmittel in Deutschland. 108

Tab. 5.4: Untersuchte Stoffe, Anwendungsbereiche und Bestimmungsgrenzen nach Nödler et al. (2010) und Reh et al. (2013)...... 118

Tab. 6.1: Systematik etablierter hydrochemischer Methoden zur hydrogeologischen Charakterisierung mit Beispielstoffen, Eintragsarten und Zielsetzungen..... 124

\section{Anhang}

Veröffentlichungen in Fachzeitschriften.................................................................................. 1

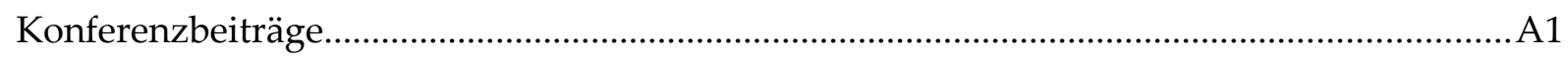

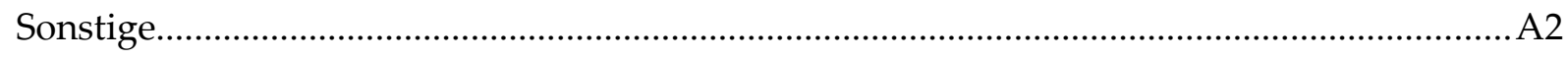

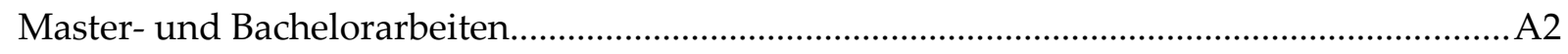

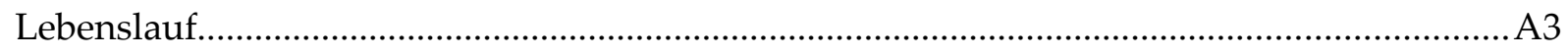





\section{Einleitung}

\subsection{Probleme und Fragestellungen der hydrogeologischen Charakterisierung von komplexen Grundwasserleiter- und Karstsystemen}

Komplexe Grundwassersysteme entstehen durch voneinander abhängige Fließbedingungen auf unterschiedlichen Geschwindigkeitsskalen, bedingt durch die Heterogenität, beispielsweise aufgrund von tektonischen Elementen (Goldscheider \& Neukum 2010) oder Klüftung (Neuman 2005). Typische Beispiele sind Karstsysteme, die auch global gesehen wasserwirtschaftlich bedeutende Frischwasservorkommen führen, da sie große Einzugsgebiete drainieren und durch Zonen hoher Durchlässigkeiten und Quellen mit großer Schüttung ergiebige Wasserentnahmen ermöglichen. Circa 25\% des weltweiten Trinkwasserbedarfs wird aus Karstsystemen gedeckt (Ford \& Williams 2007), so dass Strategien für einen effektiven Schutz solcher Ressourcen von großer Bedeutung sind. Die Entwicklung einer konzeptionellen Modellvorstellung ist essentiell für die Festlegung von Grundwasserschutzmaßnahmen (EU 2010) und als Vorstufe detaillierter Untersuchungen sowie letztendlich mathematischer Modelle unentbehrlich.

In verkarsteten Karbonatgesteinen können durch Lösungsvorgänge Trennflächen des Gesteinsgefüges bis zu Röhrensystemen und Höhlen erweitert werden. Die Folge der Verkarstungsprozesse sind Porositäten unterschiedlicher Größenordnungen, in denen Fließbewegungen mit unterschiedlichen Geschwindigkeiten und Fließbedingungen voneinander abhängen (Atkinson 1977):

1. Die Gesteinsporosität des Korngerüsts (Matrix) auf der Mikrometerskala, die durch langsame Fließbewegungen (s. Abb. 1.1) oder hydraulisch immobile Zonen charakterisiert ist, jedoch häufig einen großen Speicheranteil des Gesteins darstellt (Maloszewski 2002). Hier gelten weitgehend die hydraulischen Gesetzmäßigkeiten für poröse Medien, die sich aus der Darcy-Gleichung ableiten.

2. Kluftöffnungen (Mikrometer bis Millimeterskala) mit ebenfalls langsamen Fließbewegungen ähnlich wie in porösen Medien (White 1969, Kiraly 1998).

3. Lösungshohlräume mit teilweise großer Öffnungsweite von einigen Millimetern bis Metern. Große Hohlräume sind durch turbulente Strömungen charakterisiert und wirken als schnell abfließende Drainagen der unter 1.) und 2.) genannten Porositäten. 
Typisch für diese Hohlraumsysteme ist der Abfluss über Quellen, die große unterirdische Einzugsgebiete repräsentieren (Worthington 2003).

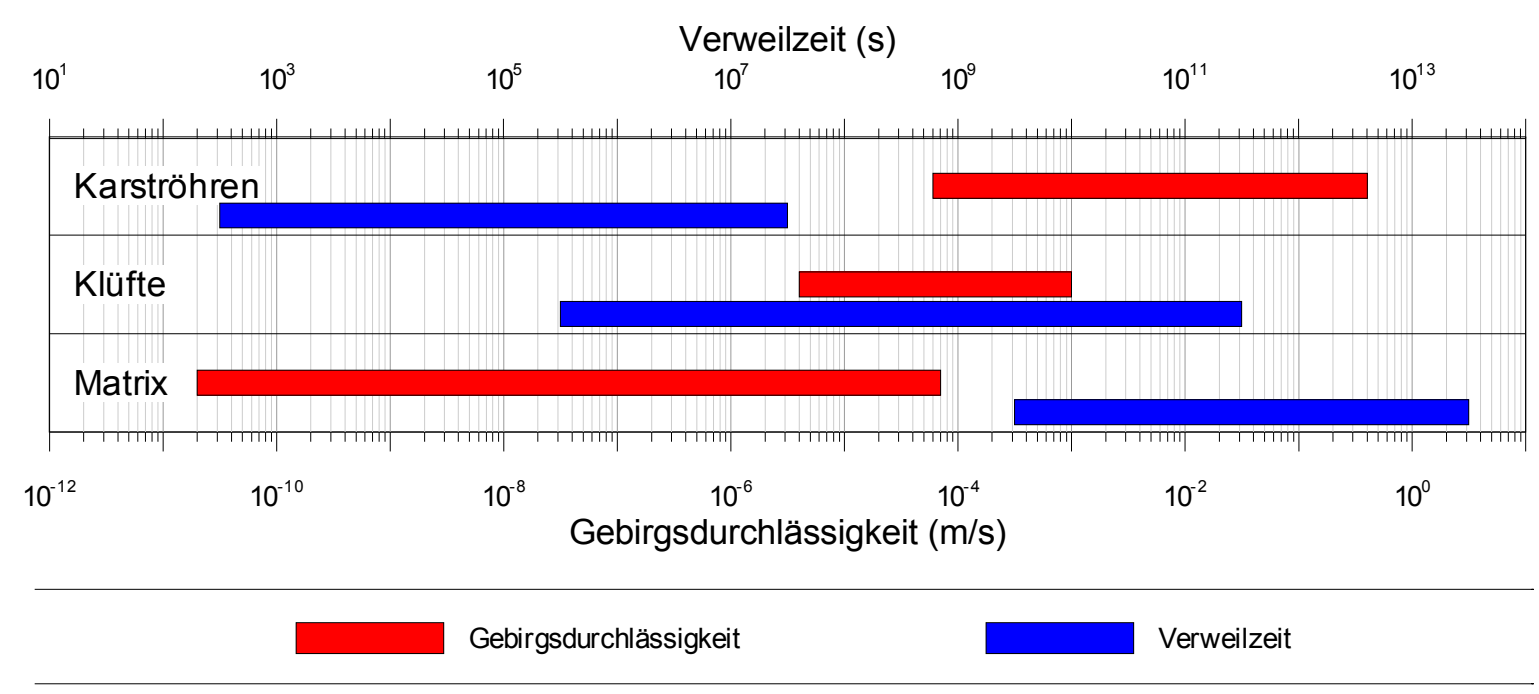

Abb. 1.1: Skalen von Gebirgsdurchlässigkeiten (nach Worthington 2003) und Verweilzeiten (nach Worthington 2007) in Karstsystemen

Diese unterschiedlichen Fließgeschwindigkeiten bedingen Wasserkomponenten unterschiedlichen Alters (s. Abb. 1.1). Sehr junges Wasser entsteht durch Neubildung mit kurzem punktuellen Eintrag in das Röhrensystem bei einem besonderen Ereignis (Starkniederschlag, Schneeschmelze). Eine längere Verweilzeit im Untergrund resultiert aus der Neubildung über die vadose Zone mit flächenhafter Infiltration über Bodenschichten. Grundwasser aus hydraulisch gering mobilen Zonen weist die höchste Verweilzeit auf (Worthington 2007).

Durch fortschreitende Lösungsvorgänge können die sich damit erweiternden unterirdischen Hohlräume ein unterirdisches Quelleinzugsgebiet ausbilden, das von den Einzugsgebieten der oberirdischen Vorfluter abweicht (Kaçaroğlu 1999, Kiraly 2003). Die Schwierigkeiten in der Beschreibung solcher Systeme resultieren vor allem aus der unbekannten räumlichen Lage und Orientierung der Röhren und Hohlräume und ihrer möglichen unterirdischen Verbindungen miteinander (Bakalowicz 2005). Ergibt sich bei guten geologischen Aufschlussverhältnissen noch die Möglichkeit, über Strukturen an der Geländeoberfläche (Dolinen, Entwässerungsstrukturen, tektonische Störungen, Kluftmuster) Hinweise auf preferentielle Fließwege im Untergrund abzuleiten, lassen sich insbesondere in urbanen Räumen und in Gebieten mit einer dichten Vegetationsdecke solche Strukturen an der Geländeoberfläche kaum detektieren. Häufig sind die Karststrukturen auch durch jüngere Deckschichten überprägt und somit an der Oberfläche nicht erkennbar (Worthington 2003). 
Basis der hydrogeologischen Charakterisierung von Karstgebieten ist die Abgrenzung der Quelleinzugsgebiete als Voraussetzung für die Abschätzung von Grundwasserneubildungsraten, Berechnungen des Grundwasserdargebots sowie die Festlegung von Schutzzonen und eines Monitoringnetzes. Darauf aufbauend ist die Ermittlung von Grundwasserfließrichtungen eines der ersten Ziele bei der Untersuchung von Grundwasservorkommen (EU 2010).

Wenn diese Grundlagen geschaffen wurden, treten zusätzliche Fragen in den Vordergrund, die auf die zeitliche Dynamik des Systems gerichtet sind. Die Abschätzung der häufig zeitlich variablen Volumenanteile (Geyer 2011, Sauter 1992) der Wasserkomponenten unterschiedlicher Verweilzeit ist ein wichtiges Instrument für die Prognose des Grundwasserdargebots, die Bewertung der Empfindlichkeit gegenüber anthropogen verursachten Kontaminationen und die Abschätzung der Auswirkung punktueller und flächenhafter Kontaminationsquellen (Daly et al. 2002).

Zur Beantwortung der oben genannten Fragestellungen werden Untersuchungsmethoden benötigt, die großräumige Zusammenhänge erfassen. Die zur Verfügung stehenden Grundwassermessstellen sind in solch großen Gebieten meistens nur in grober unregelmäßiger Verteilung vorhanden und können die Heterogenität des Systems nicht ausreichend abbilden. Daher müssen Methoden genutzt werden, die indirekt Informationen über großskalige Zusammenhänge liefern können. Jedoch mangelt es noch an Methoden, die eine Charakterisierung der Interaktion verschiedener Systeme ermöglichen (Huggenberger 2013).

\subsection{Zielsetzung dieser Arbeit}

Im zurückliegenden Jahrzehnt wurden hochempfindliche Analysenmethoden für organische Spurenstoffe entwickelt (Kolpin et al. 2002, Nödler et al. 2010), mit denen gezeigt werden konnte, dass durch menschliche Aktivität zahlreiche Stoffe freigesetzt werden (Schwarzenbach et al. 2006) und diese teilweise über lange Zeiträume in der Umwelt persistieren (Ternes 1998). Im Rahmen dieser Arbeit werden das Vorkommen und die Verteilung dieser Stoffe im Untersuchungsgebiet ausgewertet und hinsichtlich ihrer Nutzungsmöglichkeiten als Indikatoren für Fließ- und Transportprozesse im Grundwasser evaluiert. Darauf aufbauend wird gezeigt, wie in Kombination mit grundlegenden geologischen und hydrogeologischen Informationen (geologische Lagerungsverhältnisse, Grundwasserspiegel, Abflussmessungen) die Daten der organischen Spurenstoffe zum Aufbau eines konzeptionellen hydrogeologischen Modells verwendet werden können. Abschließend wird überprüft, ob die Schlussfolgerungen hinsichtlich der Nutzung organischer Spurenstoffe zur Charakterisierung von 
Grundwassersystemen im Sinne einer Generalisierung auch auf andere Gebiete anwendbar sind.

\subsection{Geologische und hydrogeologische Charakteristika des Untersuchungsgebiets}

Das Untersuchungsgebiet befindet sich im Nordwesten des Bundeslandes Hessen (Deutschland) in der sog. Waldecker-Scholle (HLUG 2008). Die drei dort vorhandenen teils verkarsteten und teils hydraulisch verbundenen Grundwasserleiter in den Karbonatformationen des Zechsteins (Karbonate der Werra-, Staßfurt- und Leine-Formation) bilden einerseits ein komplexes Fließsystem (Hölting \& Matthess 1963) und decken andererseits die lokale Trinkwasserversorgung nahezu vollständig ab.

In den Karbonatgesteinen der Werra-Formation (z1-Folge) und der Leine-Formation (z2Folge) des Zechsteins befinden sich wasserwirtschaftlich bedeutende Grundwasservorkommen, die unter anderem das Stadtgebiet Korbachs mit circa 24.000 Einwohnern mit Trinkwasser versorgen. Feinklastite der Frankenberg-Formation (zFb) sowie des Quartärs bilden lokal schwebende Grundwasserleiter über den Karbonatschichten aus. Das Stadtgebiet liegt in den Quelleinzugsgebieten der Twiste (Quellgebiet Teichmühle), der Werbe und der Itter (Quellgebiet Nieder-Ense) (Schraft et al. 2002). Die Quellgebiete der Teichmühle und Nieder-Ense sind für Karstgebiete typische Quellen mit hoher und variabler Schüttung.

Die Komplexität des Systems ist neben den Verkarstungserscheinungen und dem Grundwasserstockwerksbau durch Nord-Süd verlaufende tektonische Störungen erhöht (Kulick 1997). Durch diese Störungen bestehen lokal hydraulische Kontaktmöglichkeiten zwischen den einzelnen Grundwasserstockwerken, deren Dynamik weitgehend unbekannt ist. Näheres zum geologischen Aufbau ist den Kap. 2 - 5 zu entnehmen.

Im Stadtgebiet Korbachs emittieren mehrere bekannte Altlasten Schadstoffe (Abb. 1.2). Daneben existiert eine große Anzahl noch nicht untersuchter Verdachtsflächen. Das Grundwasser ist in Teilbereichen mit anthropogenen Schadstoffen belastet, vornehmlich leichtflüchtigen halogenierten Kohlenwasserstoffen (LHKW), so dass das im Quellgebiet der Teichmühle gewonnene Trinkwasser über Aktivkohle gefiltert werden muss. In landwirtschaftlich genutzten Gebieten führen Nitrateinträge zu erhöhten Konzentrationen im Grundwasser, denen durch ein landwirtschaftliches Beratungsprogramm gegengesteuert wird. 


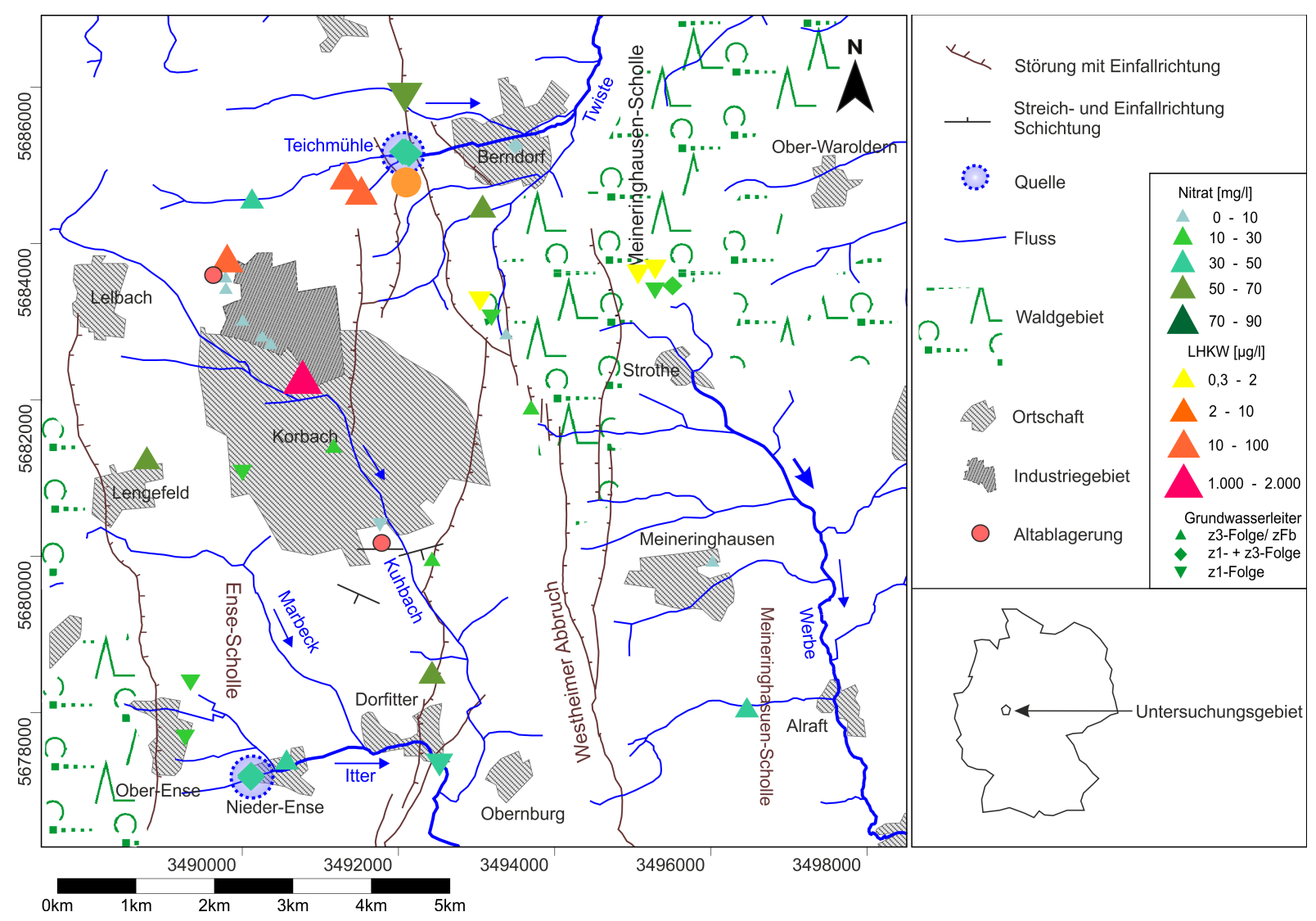

Abb. 1.2: Lage der Waldecker Scholle, Grundwasserbelastungen mit Nitrat und LHKW, Verteilung von Altflächen, Rechts- und Hochwerte als Gauß-Krüger-Koordinaten

\subsection{Aufbau der Dissertationsschrift}

Diese Dissertationsschrift ist als kumulative Dissertation verfasst, die drei veröffentlichte Artikel (Kap. 3 -5) enthält. Die Veröffentlichungen der Kap. 3 und 4 sind auf Englisch verfasst.

In den Kap. 1 und 2 werden die Fragestellungen hergeleitet sowie die Möglichkeiten und Grenzen etablierter Methoden zur Charakterisierung komplexer Grundwassersysteme diskutiert. Daran schließt sich eine thematische Einführung in das Vorkommen organischer Spurenstoffe in der aquatischen Umwelt und ihre mögliche Nutzung als Indikatoren hydrogeologischer Eigenschaften an.

Kap. 3 umfasst in Form einer Studie die Bestandsaufnahme der organischen Spurenstoffe, die im Untersuchungsgebiet untersucht wurden, sowie eine erste Auswertung der räumli- 
chen Verbreitung und Detektionshäufigkeit, teilweise in Relation $\mathrm{zu}$ den eingesetzten Mengen der untersuchten organischen Spurenstoffe.

In Kap. 4 werden diese Ergebnisse aufgegriffen und zunächst hinsichtlich ihres Potentials zur Nutzung als Indikatoren für Transportvorgänge im Grundwasser des untersuchten komplexen Karstsystems geprüft. Die räumliche und zeitliche Verteilung der Stoffe, die sich als geeignete Indikatoren herausgestellt haben, wird unter dem Gesichtspunkt der Verwendung zum Aufbau beziehungsweise zur Stützung des hydrogeologischen Standortmodells diskutiert.

In Kap. 5 wird durch zusätzliche Verwendung von Daten aus einem anderen Karstsystem (Hillebrand et al. 2012) untersucht, inwieweit sich die ermittelten Stoffspektren unterscheiden. Dieser Vergleich lässt Rückschlüsse auf den Einfluss der hydrogeologischen Eigenschaften auf das Auftreten sowie die Konzentrationen der Spurenstoffe zu. Außerdem werden Schlussfolgerungen hinsichtlich einer Generalisierung der Nutzung organischer Spurenstoffe zur Charakterisierung von Karstsystemen gezogen.

In Kap. 6 werden aus diesen drei Arbeiten zusammenfassend Schlussfolgerungen im Hinblick auf die Integration von Analysen organischer Spurenstoffe in die Untersuchungskonzepte komplexer Karstsysteme abgeleitet sowie Unterschiede zu etablierten hydrochemischen Untersuchungsmethoden und Entwicklungspotentiale aufgezeigt.

Aus der kumulativen Struktur der Dissertationsschrift ergibt sich je Kapitel ein Literaturverzeichnis.

\subsection{Literatur}

Atkinson, T.C.: Diffuse flow and conduit flow in limestone terrain in the Mendip Hills, Somerset (Great Britain). J. Hydrol. 35, 93-110 (1977)

Bakalowicz, M.: Karst groundwater: a challenge for new resources. Hydrogeol. J. 13, 148-160 (2005)

Daly, D., Dassargues, A., Drew, D., Dunne, S., Goldscheider, N., Neale, S., Popescu, I.C., Zwahlen, F.: Main concepts of the «European approach» to karst groundwater vulnerability assessment and mapping. Hydrogeol. J. 10, 340-345 (2002) 
EU: Common Implementation Strategy for the Water Framenwork Directive (2000/60/EC) Guidance Document No. 26 Guidance on Risk Assessment and the Use of Conceptual Models for Groundwater. Technical Report 042 (2010)

Ford, D.C., Williams, P.: Karst Hydrogeology and Geomorphology. 562 S.; John Wiley \& Sons Ltd., West Sussex (2007)

Geyer, T., Selg, M., Gudera, T., Sauter, M.: Langzeitabflussverhalten der Gallusquelle und des Blautopfs - relative Bedeutung der Matrix und des Karströhrensystems. Laichinger Höhlenfreund 46, 63-74 (2011)

Goldscheider, N., Neukum, C.: Fold and fault control on the drainage pattern of a doublekarst-aquifer system, Winterstaude, Austrian Alps. Acta Carsol. 39, 173-186 (2010)

HLUG: Geologische Übersichtskarte von Hessen. Wiesbaden (2008)

Hölting, B., Matthess, G.: Ein Sporentriftversuch im Zechstein von Korbach/ Hessen. Notizbl. HLfB 91, 237-255 (1963)

Huggenberger, P., Epting, J., Scheidler, S.: Concepts for the sustainable management of multiscale flow systems: the groundwater system within the Laufen Basin, Switzerland. Environ. Earth Sci. 69, 645-661 (2013)

Kaçaroğlu, F.: Review of groundwater pollution and protection in karst areas. Water Air Soil Poll. 113, 337-356, (1999)

Kiraly, L.: Karstification and groundwater flow. Speleogenesis and Evolution of Karst Aquifers 1 (2003)

Kiraly, L.: Modelling karst aquifers by the combined disrecte channel and continuum approach. Bulletin d'Hydrogeólogie 16, 77-98 (1998)

Kolpin, D.W., Furlong E.T., Meyer, M.T., Thurman, E.M., Zaugg, S.D., Barber, L.B., Buxton, H.T.: Pharmaceuticals, hormones, and other organic wastewater contaminants in U.S. streams, 1999-2000: A national reconnaissance. Environ. Sci. Technol. 36, 1202-1211 (2002).

Kulick, J.: Erläuterungen zur Geologischen Karte von Hessen 1:25.000, Blatt 4719 Korbach. 2. Aufl., 272 S.; Wiesbaden (1997) 
Maloszewski, P., Stichler, W., Zuber, A., Rank, D.: Identifying the flow systems in a karsticfissured-porous aquifer, the Schneealpe, Austria, by modelling of environmental ${ }^{18} \mathrm{O}$ and ${ }^{3} \mathrm{H}$ isotopes. J. Hydrol. 256, 48-59 (2002)

Neuman, S.P.: Trends, prospects and challenges in quantifying flow and transport through fractured rocks. Hydrogeol. J. 13, 124-147 (2005)

Nödler, K., Licha, T., Bester, K., Sauter, M.: Development of a multi-residue analytical method, based on liquid chromatography-tandem mass spectrometry, for the simultaneous determination of 46 micro-contaminants in aqueous samples. J. Chromatgr. A 1217, 6511-6521 (2010)

Sauter, M.: Quantification and forecasting of regional groundwater flow and transport in a karst aquifer (Gallusquelle, Malm, SW. Germany). 150 S.; Tübinger Geowissenschaftliche Arbeiten C13, Tübingen (1992)

Schraft, A., Fritsche, J. G., Hemfler, M., Mittelbach, G., Rambow, D., Tangermann, T.: Die hydrogeologischen Einheiten Nordhessens, ihre Grundwasserneubildung und ihr nutzbares Grundwasserdargebot (Ldkrs. Waldeck-Frankenberg, Kassel, Schwalm-Eder, WerraMeißner, Hersfeld-Rotenburg, Fulda und Stadt Kassel). Geol. Jb. Hessen 129, 27-53 (2002)

Schwarzenbach, R.P., Escher, B.I., Fenner, K., Hofstetter, T.B., Johnson, C.A., von Gunten, U., Wehrli, B.: The challenge of micropollutants in aquatic systems. Science 313, 1072-1077 (2006)

Ternes, T.A.: Occurrence of drugs in German sewage treatment plants and rivers. Water Res. 32, 3245-3260 (1998)

White, W.B.: Conceptual models for carbonate aquifers. Ground Water 7, 15-21 (1969)

Worthington, S.R.H.: A comprehensive strategy for understanding flow in carbonate aquifer. Speleogenesis and Evolution of Karst Aquifers 1(1) (2003)

Worthington, S.R.H.: Groundwater Residence Times in Unconfined Carbonate Aquifers. Journal of Cave and Karst Studies 69, 94-102 (2007) 


\section{Charakterisierung großräumiger komplexer Grundwassersysteme und Karstgrundwasserleiter}

\subsection{Etablierte Erkundungsmethoden}

\subsubsection{Hydrogeologische Methoden}

\subsubsection{Erkundung der geologischen Lagerungsverhältnisse und tektonischen}

\section{Strukurelemente}

Grundwasserfließrichtungen in Kluft- und Karstsystemen werden zum einen durch die hydraulischen Potentiale und zum anderen durch die geologischen Lagerungsverhältnisse und Strukturelemente bestimmt (Goldscheider \& Neukum 2010, Herold et al. 2000). Daher lassen Höhenbeziehungen sowie Kenntnisse der geologischen Strukturen und Gefügelemente, wie die Einfallsrichtung der grundwasserführenden Schichten, die Ausrichtung von Kluftöffnungen oder die Lage von Störungszonen Rückschlüsse auf die möglichen Grundwasserfließrichtungen und die Lage von unterirdischen Einzugsgebietsgrenzen zu (Ashjari \& Raeisi 2006, Goldscheider \& Neukum 2010, Kiraly 2003). Die Erkundung der geologischen Verhältnisse ist somit ein elementarer Bestandteil der hydrogeologischen Erkundung. Jedoch können insbesondere in Abfolgen von Karbonatgesteinen die Grundwasserfließbewegungen unabhängig von stratigraphischen Grenzen stattfinden (Sauter 1992). Störungszonen können als drainierende Elemente aber auch als hydraulische Barrieren fungieren (Babiker \& Gudmundsson 2004). Daher sind in der Regel weitergehende Informationen über die hydraulische Funktion der geologischen Strukturelemente erforderlich.

\subsubsection{Hydraulische Methoden}

Messungen der Grundwasserstände und ihre Interpolation in Form von Grundwassergleichenplänen zur Bestimmung der Grundwasserfließrichtung stellen in der Regel die Basis flächenhafter hydrogeologischer Erkundungen dar (Hölting \& Coldewey 2013). In Karstgebieten sind die Wasserspiegelhöhe und die zeitliche Dynamik stark davon abhängig, ob die Messstelle in beziehungsweise in unmittelbarer Nähe zu einer bevorzugten Drainagestruktur (Karströhre) oder innerhalb der klüftigen Gesteinsmatrix liegt (Worthington 2009). Die Unkenntnis der Lage bevorzugter Drainagestrukturen führt daher zu ungenauer oder fehlerhafter Interpretation der Grundwasserfließrichtung (Taylor \& Greene 2008). Insbesondere in 
Karstgebieten wäre folglich ein engmaschiges Messnetz erforderlich, das aber aufgrund des hohen Aufwands häufig nicht realisiert werden kann.

Für grundlegende Berechnungen von Massenflüssen und Fließgeschwindigkeiten werden hydraulische Parameter (Transmissivität/ Gebirgsdurchlässigkeit, Porosität, Speicherkoeffizient) benötigt, die üblicherweise mit Bohrlochtests, wie hydraulische Pumpversuche oder andere geophysikalische Bohrlochmessungen, bestimmt werden. Diese punktuellen Untersuchungen repräsentieren nur einen räumlich begrenzten Ausschnitt des Grundwassersystems (Larocque et al. 2009), der die Heterogenität mit den unterschiedlich skalierten Porositäten und Fließgeschwindigkeiten eines Karstgrundwasserleiters nicht ausreichend repräsentiert. Großflächige heterogene Systeme erfordern einen hohen Aufwand durch ein dichtes Netz an Punktinformationen (Bohrlöchern) oder durch die Entnahme sehr großer Volumina über Pumpversuche (Maréchal et al. 2008).

Um diesem Aufwand zu entgehen, hat sich in Karstsystemen die Interpretation von Ganglinien der Quellschüttungen (Abflusskurven) etabliert, die auf der Modellvorstellung beruhen, dass eine Karstquelle an eine Drainagestruktur (Karströhre) angeschlossen ist und dadurch Eigenschaften des Gesamtsystems inklusive der klüftigen Gesteinsmatrix repräsentiert (Liedl et al. 2003). Auswertungen von Abflussmessungen dienen als wesentliche Grundlage zur Bestimmung der Grundwasserneubildungsrate oder des Speichervolumens auf Grundlage der bekannten Fläche des Einzugsgebiets nach Abtrennung einer oberirdischen von einer unterirdischen Abflusskomponente mittels graphischer oder statistischer Verfahren (Maillet 1905, Richter \& Lillich 1975, Wundt 1958).

Der Abfluss von Karstquellen besteht nur aus unterirdischen Abflusskomponenten, die sich aus langsamen und schnellen Fließkomponenten zusammensetzen und sich in der Quellschüttungsganglinie widerspiegeln (Geyer et al. 2013, Sauter 1992). Damit erlauben Quellschüttungsganglinien ähnlich hydraulischen Pumpversuchen auch Interpretationen der Speichereigenschaften und hydraulischen Durchlässigkeiten (Kovács et al. 2005). In gut entwickelten Karstsystemen wird der rezessive Verlauf der Basisabflusskurve durch die hydraulischen Parameter der Gesteinsmatrix dominiert, während in gering entwickelten Karstsystemen die Basisabflusskurve maßgeblich durch die Geometrie der gering durchlässigen Blöcke, die Durchlässigkeit des Röhrensystems sowie das Volumen des Grundwasserleiters beeinflusst wird (Kovács et al. 2005). Einzelne Abschnitte einer Abflusskurve repräsentieren nicht nur Systeme unterschiedlicher Durchlässigkeiten sondern auch verschiedene Block- oder Aquifergeometrien (Fiorillo 2014, Kovacs \& Perrochet 2008). Birk \& 
Hergarten (2010) charakterisieren die Geometrie des Röhrennetzwerks und der Matrixblöcke mittels des frühen Verlaufs der Quellschüttungsganglinie. Erweiterte Analysen des Kurvenverlaufs lassen eine Abtrennung des Anteils der direkten Grundwasserneubildung durch Bildung von Ableitungsfunktionen zu (Geyer et al. 2008). Zusätzliche Informationen zur Schüttungsganglinie hinsichtlich der Quantifizierung von Grundwasserneubildungsereignissen kann die begleitende Auswertung von Chemographen physikochemischer Parameter (beispielsweise elektrische Leitfähigkeit, Trübe) oder der gelösten Ionen bereitstellen (Doctor et al. 2006, Pronk et al, 2009).

Die Auswertung von Schüttungsganglinien zielt auf die Ermittlung der Grundwasserneubildungsrate, auf die Identifizierung von Wasserkomponenten unterschiedlicher Verweilzeit und, ebenso wie hydraulische Tests, auf die Ermittlung hydraulischer Parameter ab. Hiermit lässt sich die Ausdehnung eines Einzugsgebiets nur indirekt ermitteln, wenn gleichzeitig weitere Parameter der Wasserbilanz (Niederschlagsmenge, Evapotranspiration) ermittelt werden.

\subsubsection{Vulnerabilitätskartierungen}

Zur hydrogeologischen Charakterisierung eines Karstsystems und sich daraus ableitenden Schlussfolgerungen zum Grundwasserschutz sind neben Kenntnissen über die hydraulischen Zusammenhänge, Fließrichtungen und Fließgeschwindigkeiten auch Kenntnisse über die Verschmutzungsempfindlichkeit (intrinsische Vulnerabilität) erforderlich (COST 2003). Hierfür werden im Allgemeinen die Grundwasserneubildung und die Schutzwirkung der Deckschichten des Grundwasserleiters bewertet (Hölting et al. 1995). Aufgrund der Heterogenität von Karstsystemen wurden erweiterte Methoden entwickelt, die eine schnelle Neubildungskomponente (Goldscheider et al. 2000, Daly et al. 2002) und das Karströhrennetzwerk (Dörflinger \& Zwahlen 1998) berücksichtigen. Die Bewertung ist stets indirekt und birgt die Gefahr, dass kleinräumige und heterogene Strukturen mit abweichender Schutzwirkung nicht berücksichtigt werden. Eine Validierung der interpretierten Karten erhöht daher deren Zuverlässigkeit. Hierfür werden unter anderem hydrochemische Daten, insbesondere anthropogen eingetragener Stoffe, interpretiert (Neukum 2013). 


\subsubsection{Nutzung hydrochemischer Daten zur Charakterisierung von Grundwassersystemen}

\subsubsection{Basiskonzepte}

Grundlage der Nutzung hydrochemischer Methoden für jegliche Charakterisierung von Grundwassersystemen ist die Detektion zeitlich oder räumlich variabler Konzentrationen oder Intensitäten, die hinsichtlich der jeweiligen Fragestellung interpretiert werden. Die Untersuchungsstrategien umfassen aktive Verfahren, die die Eingabe eines Stoffes zum Zweck der Untersuchung vorsehen und passive Methoden, die bereits im Grundwasser vorhandene Stoffe nutzen.

Zur Gruppe der aktiven Verfahren gehören die häufig angewandten Tracer-/ Markierungsversuche mit gelösten zumeist fluoreszierenden Farbstoffen, Salzen oder gefärbten Feststoffpartikeln (Käss et al. 2004). Ein Charakteristikum der künstlichen Markierungsversuche ist die Klärung hydrogeologischer Punkt-zu-Punkt-Beziehungen in schnell fließenden Systemen (Goldscheider et al. 2008). Da Karstsysteme durch die Interaktion einer schnellen und einer langsamen Fließkomponente gekennzeichnet sind, wird durch die künstlichen Markierungsstoffe ein erheblicher Teil des Fließgeschehens nicht abgebildet (Doctor et al. 2006). Die Erkundung der langsam fließenden Komponente mit Verweilzeiten von Jahren, Jahrzehnten oder noch längerer Zeiträume erfordert Methoden, die Stoffe nutzen, die weit vor dem Untersuchungszeitpunkt in das Grundwasser eingetragen wurden und deren Vorkommen an das langsame Fließkomponente gebunden ist (Cook \& Solomon 1997).

Die Palette der hydrochemischen Methoden umfasst Bestimmungen der Hauptionenzusammensetzung (Furtak \& Langguth 1967, Piper 1944, Schoeller 1955), stabiler und radioaktiver Isotope (Etcheverry \& Vennemann 2009, Sidle 1998), natürlich vorkommender anorganischer Spurenstoffe (Dia et al. 2000, Siebert et al. 2012) sowie anthropogen eingetragener Kontaminanten (Cook \& Böhlke 2000). Wichtige Unterschiede bestehen zwischen Methoden, die natürlich vorkommende Stoffe nutzen und Verfahren, die auf der Anwendung anthropogen freigesetzter Stoffe beruhen. Erstgenannte umfassen die Hauptionen, natürlich gebildete Isotopenverhältnisse und Metalle der Seltenen Erden. Sie erlauben im Gegensatz zur Anwendung anthropogen eingetragener Stoffe auch die Untersuchung von Wasserkomponenten, die vor Beginn des industriellen Zeitalters gebildet wurden.

$\mathrm{Zu}$ unterscheiden sind weiterhin Methoden mit Stoffen bekannter Eintragsfunktion von Methoden mit Stoffen unbekannter Eintragsfunktion. Die meisten Anwendungen von 
Isotopen setzen eine bekannte Eintragsfunktion voraus. Diese ist bei Versuchen mit künstlichen Markierungsstoffen bereits per Definition gegeben. Die Verteilungen und Verhältnisse der gelösten Hauptionen, der Metalle der Seltenen Erden und von Umwelttracern können je nach Fragestellung auch ohne bekannte Eintragsfunktion interpretiert werden (Furtak \& Langguth 1967, Johannesson 1997, Sültenfuss \& Massmann 2004). Im Folgenden werden Stoffe, deren Eintragsfunktion bekannt ist, als „Tracer-/ Markierungsstoffe“ bezeichnet, während Stoffe, deren Eintragsfunktion unbekannt ist oder nur grob abgeschätzt werden kann, „Indikatoren“ genannt werden. Stoffe bekannter Eintragsfunktion, die nicht mit der Absicht der geologischen Erkundung freigesetzt wurden, sind „Umwelttracer“.

Sowohl die aktiven Markierungsverfahren als auch die passiven Methoden umfassen Stoffe, die weitgehend konservativ ohne chemische, biologische oder physikalische Rückhalteprozesse mit der Grundwasserströmung transportiert werden (Käss et al. 2004) als auch reaktive Stoffe, deren Konzentrationen auf der Fließstrecke durch Attenuationprozesse gemindert werden (Geyer et al. 2007). Die Vorhersage der Ausbreitung von Schadstoffen beziehungsweise die Angabe von Retardationsfaktoren basiert zumeist auf dem Verhältnis der Geschwindigkeit reaktiver Markierungsstoffe zur Geschwindigkeit konservativ transportierter Stoffe (Geyer et al. 2007).

\subsubsection{Versuche mit künstlichen Markierungsstoffen}

Künstliche Markierungsstoffe werden aktiv an einer definierten Eingabestelle mit bekannter Eintragsfunktion (Menge je Zeiteinheit) in den Grundwasserleiter eingegeben und ihre Konzentration an einer oder mehreren Auffangstellen quantitativ analytisch bestimmt (Käss et al. 2004). Diese Punkt-zu-Punkt-Beziehungen lassen rein qualitativ exakte Bestimmungen der Grundwasserfließrichtungen zu und können beispielsweise gezielt eingesetzt werden, um Fließwege im Untergrund oder die Zugehörigkeit von Eingabestellen zu einem Einzugsgebiet zu überprüfen (Goldscheider 2005). Häufig eingesetzt werden Fluoreszenzfarbstoffe (beipielsweise Uranin, Eosin, Rhodmine, Tinopal CBS-X), Salze (beispielsweise Chloride oder Bromide) oder fluoreszierende Partikel (microspheres) (Goldscheider 2008, Käss \& Behrens 2004). Heutige Anwendungen gehen über diese rein qualitativen Auswertungen hinaus und umfassen in Systemen mit bekannten Fließrichtungen auch die Quantifizierung hydrogeologischer Parameter, wie Fließgeschwindigkeit, Dispersivität sowie Röhrenvolumen und Durchmesser, mittels der Auswertung der Durchbruchskurven der Markierungsstoffkonzentrationen an einer Beprobungsstelle durch analytische (Field 1999, Field \& Pinsky 2000) oder numerische Modelle (Birk et al. 2005). Ein Beispiel für einen Markierungsversuch mit qualitativer Auswertung findet sich im Untersuchungsgebiet selber. Im Mai 1962 wurde 
von Hölting \& Matthess (1963) ein sog. „Sporentriftversuch“ mit unterschiedlich gefärbten Bärlappsporen (Durchmesser von rund $30 \mu \mathrm{m}$ ) in 5 Eingabestellen durchgeführt. Aufgabenstellung war seinerzeit die Vorerkundung möglicher Standorte für Mülldeponien. Die Sporen wurden in den Beobachtungsmessstellen mittels aus Seidengaze gefertigen Planktonnetzen aufgefangen, womit eine quantitative Erfassung nicht möglich war. Da innerhalb der zeitlichen Auflösung der Beprobung (Austausch der Netze) von rund 1-2 Tagen einzelne bis max. einige Zehner Sporen aufgefangen wurden, kann man aus heutiger Sicht nur von einem Spurenbefund sprechen.

Künstliche Markierungsversuche können in großräumigen Zusammenhängen zumeist aus logistischen und wirtschaftlichen Gründen nur für die Untersuchung schneller präferentieller Fließwege beispielsweise innerhalb größerer Hohlräume und Klüfte eingesetzt werden, die eine Versuchsdurchführung innerhalb von wenigen Tage bis max. einigen Wochen zulassen. Langsame Fließkomponenten werden folglich durch künstliche Markierungsversuche zumeist nicht erfasst. Weiterhin ist für die Durchführung eine Vorkenntnis des Systems erforderlich, da sowohl geeignete Eingabestellen als auch Beobachtungsmessstellen vorhanden sein beziehungsweise eingerichtet werden müssen. Als Eingabestellen werden bevorzugt Verkarstungsstrukturen wie Dolinen, erweiterte Klüfte oder Bachschwinden verwendet, von denen eine Anbindung an einen präferentiellen Fließweg anzunehmen ist (Goldscheider et al. 2008). Dadurch sind die Einsatzmöglichkeiten in Stadtgebieten aufgrund der Überbauung von potentiellen Eingabestellen und den Ausbau von Bachläufen stark limitiert. Ersatzweise herangezogene Grundwassermessstellen erschließen zumeist die feingeklüftete Gesteinsmatrix (Kiraly 2003) und führen daher nicht zu zufriedenstellenden Ergebnissen. Bei aktuellen Probenahmen im Rahmen dieser Studie zeigte der innerhalb einer Messstelle abgelagerte Schlamm, dass ein Großteil des im Jahr 2002 eingegebenen Fluoreszenzfarbstoffs Sulforhodamin noch 8 Jahre nach der Eingabe in der Messstelle vorhanden war. Dieser Fund weist auch darauf hin, dass durch vorherige Markierungsversuche eine Hintergrundkonzentration entstehen kann. Weiterhin ist bekannt, dass die Detektion der Fluoreszenztracer durch eine natürliche Hintergrundfluoreszens durch organische Kohlenstoffverbindungen (Baker \& Lamont-Black 2001) als auch durch eine anthropogen verursachte Fluoreszenz durch Freisetzungen aus Altablagerungen (Baker \& Curry 2004) gestört werden kann. 


\subsubsection{Interpretation gelöster Hauptionen}

Seit Jahrzehnten bekannte Methoden sind die Typisierung von Grundwässern verschiedener Herkunft oder unterschiedlicher Genese auf Grundlage der Verhältnisse der gelösten Hauptionen. (Piper 1944, Schoeller 1955). Hierfür wird der beim Durchströmen chemisch unterschiedlicher Boden- und Gesteinsschichten entstehende Kontrast der gelösten Stoffe genutzt. Erweiterungen dieser Methode nutzen ergänzende Verhältnisdiagramme (Furtak \& Langguth 1967) oder berücksichtigen zusätzlich die Gesamtkonzentration der gelösten Stoffe (d'Amore et al. 1983).

Verhältnisse und Konzentrationen der Hauptionen können genutzt werden, um eine aus intensiven Grundwasserbildungsereignissen resultierende schnelle Fließkomponente von einer langsamen Komponente zu unterscheiden (Pinault et al. 2001). Toran \& Reisch (2013) zeigen dieses für Ca/Mg-Verhältnisse, während Mahler \& Garner (2009) Nitratkonzentrationen nutzen. Vorteil dieser Methoden ist die mit etablierten chemischen Analysenmethoden einfache Anwendung. Entsprechend finden sich viele Studien, die die Hauptionenzusammensetzung nutzen, beispielsweise Doctor et al. (2006), Knowles et al. (2010), Stüber \& Criss (2005), Toth \& Katz (2006) und andere, zumeist in Kombination mit anderen Methoden.

Grundvoraussetzung ist jedoch die Entstehung eines signifikanten Kontrasts der gelösten Ionen oder der Verhältnisse der gelösten Ionen zueinander, die auf die unterschiedlichen chemischen Gesteinseigenschaften zurückgeführt werden kann (Jahnke 1999) oder auf unterschiedliche Verweilzeiten (Barbieri et al. 2005). Die Anwendbarkeit ist folglich in den Gebieten stark eingeschränkt, in denen aufgrund der chemischen Ähnlichkeit der durchströmten Gesteine kein Kontrast der Hauptinhaltsstoffe entsteht. Dieses ist in Karbonat- und Karstgrundwasserleitern insbesondere dann der Fall, wenn das gesamte Einzugsgebiet aus lithologisch einheitlichen Formationen besteht (Terzić et al. 2012). In besiedelten Gebieten können die ursprünglichen Ionenkonzentrationen durch zahlreiche anthropogene Einflüsse, wie beispielsweise Bodenkontaminationen, Streusalzeinträge oder Kanalleckagen, beeinflusst sein. Diese stellen dann Verschmutzungsindikatoren dar. Scanlon (1990) kann eine Korrelation mit dem Auftreten von Bakterien herstellen. Durch landwirtschaftlich eingesetzte Produkte werden die natürlichen Konzentrationen von Natrium, Kalium, Stickstoff, Fluor und Phosphor verändert (Stueber \& Criss 2005). Einzelne Ionen sind reaktiv (Nitrat, Sulfat, Eisen, Mangan, Hydrogenkarbonat) und können in unterschiedlichen Oxidationsstufen vorliegen, die möglicherweise nicht vollständig erfasst werden. In diesen Fällen geben die Grundwasseranalysen nicht mehr die Charakteristik der grundwasserleitenden Schichten 
wieder. Mittels hydrochemischer Modellierungssoftware können Reaktionsprozesse innerhalb des Grundwasserleiters bei der Auswertung berücksichtigt werden (Barbieri et al. 2005).

\subsubsection{Interpretation von Signaturen der Metalle der Seltenen Erden und Yttrium}

Gesteine unterschiedlicher genetischer Herkunft weisen charakteristische Muster von Lanthanum (La), der 14 Metalle der Seltenen Erden und Yttrium (REY) auf, die aus der Fraktionierung bei der Mineral- und Lithogenese resultieren (Hanson 1980). Zur Verdeutlichung dieser Fraktionierung werden die ermittelten Konzentrationen auf eine Standardverteilung bezogen. In vielen Fällen wird ein Chondritstandard (Hooker et al. 1976) oder ein Tonschiefer-Standard (Post Archean Australian Shale: PAAS) verwendet (Taylor \& McLennan 1985).

Studien der 1990er Jahre (Smedley 1991, Johannesson 1997) konnten zeigen, dass REY-Signaturen in Grundwässern charakteristisch für deren hydrogeologische Herkunft sind. Fallbeispiele weisen darauf hin, dass REY-Signaturen potentiell als Indiktoren hydrogeologischer Zusammenhänge geeignet sind: Beispielsweise lassen sich Zonen der Grundwasserneubildung voneinander unterscheiden, die zum einen durch anstehende Kalksteine und zum anderen durch Basalte charakterisiert sind (Siebert et al. 2012). Nach Dia et al. (2000) können Grundwässer tiefer gelegener proterozoischer Schiefer von denen in der darüber liegenden Verwitterungsdecke unterschieden werden. Tweed et al. (2006) detektieren Komponenten von Mischwässern eines Grundwasserleiters vulkanischer Genese und eines Grundwasserleiters innerhalb silurisch-devonischer Sedimentgesteine. In den genannten Beispielen liegen deutliche chemische Unterschiede der wasserleitenden Gesteinsschichten vor, die sich auch in starken Kontrasten der Hauptionenverhältnise ausdrücken.

Trotz der potentiellen Nutzbarkeit als Indikatoren sind aktuelle Studien in komplexen Grundwasserleitern und Karstsystemen selten. Ein Grund hierfür liegt darin, dass die Konzentrationen der REY-Metalle in der aquatischen Umwelt unter anderem pH-Wert abhängig sind, an die organische Kolloidfraktion sorbieren (Janssen \& Verweij 2003) und bei ihrer Interpretation die den hydrochemischen Bedingungen korrespondierenden Bindungsformen berücksichtigt werden müssen (Janssen et al. 2003). Gerade in schwach basischen Grundwässern und Oberflächengewässern, die typisch für Karstgebiete sind, wurden vergleichsweise geringe REY-Konzentrationen gemessen (Han \& Liu 2007, Shouyang et al. 2011). Diese Eigenschaften und der häufig geringe chemische Kontrast in Karstsystemen hindern die Nutzung von REY zur Charakterisierung solcher Systeme. Die mögliche Nutzung wird weiterhin eingeschränkt durch anthropogen verursachte Eingriffe in das Verteilungsmuster der REY durch Bergbauhalden (Grawunder et al. 2014), Verwendung in 
elektronischen Produkten und industriellen Prozessen (Kulaksız \& Bau 2013) sowie in medizinischen Produkten, wie beispielsweise die Verwendung von Gadolinium (Gd) in Kontrastmitteln für die Magnetresonanztomographie (MRT) (Bau \& Dulski 1996).

\subsubsection{Auswertung der Fraktionierung natürlich vorkommender Isotope}

Ungefähr 1.700 verschiedene Isotope gelöster Stoffe oder Gase natürlichen Ursprungs kommen in der aquatischen Umwelt vor (Sidle 1998) vor. Sie stellen damit eine relativ große Gruppe potentieller Tracer oder Indikatoren. Die Nutzung zur Charakterisierung hydrogeologischer Eigenschaften beruht in der Regel auf der Veränderung des Verhältnisses der Isotopen eines Elements zueinander. Bei stabilen Isotopen ist diese Fraktionierung allein eine Folge physikalischer, chemischer und biologischer Prozesse wie Phasenübergänge oder biologischer Abbau (Sidle 1998). Bei radiokativen Isotopen ergibt sich eine zusätzliche Fraktionierung durch den radioaktiven Zerfall (Kendall \& McDonnell 1998, Gat 2010). Weitere wichtige Unterschiede bestehen in der Form, in der die Isotope im Grundwasser vorliegen. Sauerstoff- und Wasserstoffisotope sind als Bestandteile des Wassermoleküls im Grundwasser bei niedrigen Temperaturen insofern konservative Tracer, dass sie nicht mit dem Material des Grundwasserleiters reagieren. Dennoch kann auch im Grundwasser eine weitere Fraktionierung erfolgen, beispielsweise durch Evaporation oder Kondensation im Austausch mit der ungesättigten Zone (Sidle 1998). Die Isotopenverhältnisse gelöster Stoffe können hingegen durch Reaktionen mit anorganischen oder organischen Materialien des Grundwasserleiters verändert werden(Sidle 1998, Barbieri et al. 2005). Die Verhältnisse der natürlich vorkommenden stabilen Isotope des Wassermoleküls $\left({ }^{2} \mathrm{H}=\right.$ Deuterium $/{ }^{1} \mathrm{H}$ und ${ }^{16} \mathrm{O} /{ }^{18} \mathrm{O}$ ) werden in der Regel als Abweichung von einem Standard ( $\delta$-Werte) gemessen und angegeben (IAEA 1981). Jeweils bei Phasenübergängen (1. Verdunstung, 2. Kondensation, 3. Niederschlag) verschieben sich die Verhältnisse (Dansgard 1964). Im Grundprinzip gehen die leichteren Isotope bevorzugt in die Gasphase über. Diese Fraktionierung führt zu einer relativen Anreicherung der schwereren Isotope in der verbliebenen Flüssigphase. Auf der anderen Seite kondensieren die schwereren Isotope bevorzugt (Etcheverry \& Vennemann 2009). Das Verhältnis zwischen schweren und leichten Isotopen im Niederschlagswasser wird von der Temperatur und damit auch von der orographischen Höhe und von der geographischen Lage (Craig 1961, Stumpp et al. 2014) beeinflusst. Die Auswertung der stabilen Isotope des Wassermoleküls beruht zumeist auf dem Kontrast zwischen der aktuellen lokalen Isotopenfraktionierung im Regenwasser und den Isotopenverhältnissen im Grundwasser. Mittels vereinfachender zusammenfassender Funktionen (sog. lumped Parametermodelle) können Rückschlüsse auf die Grundwasserneubildungsbedingungen wie 
Temperatur und Höhenlage des Grundwasserneubildungsgebiets gezogen werden (Maloszewski \& Zuber 1996).

Eine verbreitete Anwendung in komplexen Grundwasser- und Karstsystemen ist die Bestimmung der Volumenanteile unterschiedlicher Fließkomponenten. So wird beispielsweise von Long \& Putnam (2004) das Verhältnis von ${ }^{18} \mathrm{O} /{ }^{16} \mathrm{O}$ zur Abschätzung von Grundwasserfließgeschwindigkeiten, Verweilzeiten und der Mengenanteile einzelner Fließkomponenten in einem Karstgrundwasserleiter mit punktuellem Einfluss durch einen infiltrierenden Fluss genutzt. Peng et al. (2014) können die Beziehung zwischen Grund- und Oberflächenwasser in mächtigen alluvialen Fansedimenten mit mehreren Grundwasserstockwerken klären sowie eine Vorstellung hinsichtlich der Gebiete der Grundwasserneubildung entwickeln. Auch Murgulet \& Tick (2013) nutzen stabile Isotope zur Untersuchung der Interaktion von verschiedenen Grundwasserleitern. Sun et al. (2014) zeigen, dass der Einfluss von bergbaulichen Sickerwässern in einem Karstsystem mittels stabiler Isotope identifiziert werden kann. Eine weitere mögliche Anwendung ist die Validierung von Vulnerabilitätskartierungen (Dimitriou \& Zacharias 2006) durch die Abschätzung von neu gebildeten Grundwasseranteilen gegenüber einer älteren Komponente.

Die Abgrenzung von Quelleinzugsgebieten ist mittels der Höhenbeziehung der stabilen Isotope möglich (Barbieri et al. 2005), setzt jedoch für eine identifizierbare Fraktionierung ein deutliches Relief des Untersuchungsraums voraus. Der notwendige Kontrast der Isotopenverhältnisse kann in Gebieten ohne ausgeprägtes Geländerelief unter Berücksichtigung des saisonalen Jahresgangs entweder durch kurzzeitige Ereignisse - beispielsweise Starkniederschläge, Schneeschmelzen - oder über lange Zeiträume durch Änderungen der klimatischen Verhältnisse entstehen. Ab einer Verweilzeit im Grundwasser von ungefähr 4 Jahren wird das jahreszeitliche Signal der ${ }^{18} \mathrm{O}$-Signaturen gedämpft (Stichler \& Hermann 1983). Wird eine Wasserkomponente innerhalb eines klimatisch einheitlichen Zeitraums gebildet und ist keine saisonal davon abweichende Kurzzeitkomponente vorhanden, ist aufgrund des mangelnden Kontrasts eine Nutzung zur Charakterisierung von Fließkomponenten folglich kaum möglich. Um die beschränkten Nutzungsmöglichkeiten einzelner Isotope zu erweitern, werden Studien häufig in Kombination mit Isotopenverhältnissen gelöster Stoffe, wie beispielsweise Strontium (Barbieri et al. 2005), oder vorwiegend anthropogen gebildeter Isotope, wie Tritium (Maloszewski 2002), durchgeführt.

Neben den stabilen Isotopen des Wassermoleküls können auch stabile Isotope gelöster oder gasförmiger Stoffe zur hydrogeologischen Charakterisierung genutzt werden. Im Unter- 
schied zu den Isotopen des Wassermolelüls werden die Verhältnisse gelöster Stoffe, wie beispielsweise die der Strontiumisotope, durch chemische, physikalische oder biologische Prozesse auf dem unterirdischen Fließweg verändert (Åberg 1994). Die Strontiumisotope ${ }^{87} \mathrm{Sr} /{ }^{86} \mathrm{Sr}$ kommen in natürlichen Grundwässern in relativ hohen Konzentrationen vor. Ihr Verhältnis ist aufgrund der geringen Massendifferenzen im Verhältnis zur Molmasse relativ robust gegenüber Evaporations- und Transpirationsprozessen (Paces \& Wurster 2014). Da das Verhältnis im Wesentlichen durch atmosphärisches $\mathrm{CO}_{2}$ und den Karbonatgehalt des Aquifermaterials bestimmt wird, unterscheidet sich die Signatur einer neu gebildeten Komponente von einer Komponente mit längerer Verweilzeit (Emblanch et al. 2003). Damit sind Strontium-Isotope in Karstsystemen beispielsweise für die Abschätzung des Neubildungsanteils im Grundwasserleiter nutzbar (Barbieri et al. 2005).

Das radioaktive Isotop des Edelgases Argon $\left({ }^{39} \mathrm{Ar}\right)$ ist hingegen nicht reaktiv und wird für Altersbestimmungen im Bereich von $>50$ Jahren bis zu Jahrhunderten genutzt (Loosli 1983). Durch die Nutzung des Verhältnisses zum stabilen Argon-Isotop ${ }^{40} \mathrm{Ar}$ ist die Methode unanfällig bei auftretenden Ausgasungsverlusten in der Quelle oder bei der Probenahme und daher für Karstsysteme gut geeignet. Bisher sind keine anthropogenen Quellen für ${ }^{39} \mathrm{Ar}$ bekannt (Delbart et al. 2014). Beispiele zur Bestimmung langer Verweilzeiten mehrerer 1.000er bis 10.000er Jahre finden sich für ${ }^{14} \mathrm{C}$ (Radiokarbonmethode) (Gonfiantini et al. 1998, Murgulet \& Tick 2013) und für Uran (Suksi et al. 2006, Paces \& Wurster (2014).

Weitere Anwendungen gelöster stabiler Isotope sind die Identifizierung biologischer und chemischer Prozesse, beispielsweise mittels der ${ }^{13} \mathrm{C} /{ }^{12} \mathrm{C}$ und ${ }^{15} \mathrm{~N} /{ }^{14} \mathrm{~N}$ Verhältnisse (Katz et al. 2004) oder die Unterscheidung von Quellwässern mittels des ${ }^{13} \mathrm{C}$-Anteils am gesamten anorganischen Kohlenstoff (DIC) (Tallini et al. 2014). Die Verhältnisse der vier natürlich vorkommenden stabilen Schwefelisotope werden ebenfalls zur Identifizierung chemischbiologischer Prozesse genutzt (Sueker 2003, Sack \& Sharma 2014).

\subsubsection{Interpretation der Konzentrationen und Intensitäten von Umwelttracern}

Cook \& Böhlke (2000) definieren „Umwelttracer" als Stoffe natürlicher und anthropogener Herkunft, die in der Umwelt eine weite Verbreitung haben. In der im Folgenden zitierten Literatur wird der Begriff „Umwelttracer" nahezu ausschließlich für anthropogen freigesetzte Stoffe verwendet. Da die meisten von ihnen unter atmosphärischen Druckverhältnissen gasförmig sind, werden sie zumeist über Niederschläge aus der Atmosphäre in das Grundwasser eingetragen. Typische Umwelttracer sind radioaktive Isotope oder auch organische Stoffe. Gemeinsames Kennzeichen ist eine bekannte Eintragsfunktion durch 
Messungen der Konzentration beziehungsweise Aktivität im Niederschlag. Ein etablierter Umwelttracer ist das Wasserstoffisotop Tritium $\left({ }^{3} \mathrm{H}\right)$, das nur in sehr geringen Mengen natürlich vorkommt (Rozanski et al. 1991) und bis in die 1960er Jahre durch Atomwaffentests in größeren Mengen in der Atmosphäre gebildet wurde (Brown et al. 1975). Ebenso wie das stabile Wasserstoffisotop Deuterium ist es Bestandteil des Wassermoleküls. Ein zunehmendes Problem der Anwendung der Tritiummethode ist die Konzentrationsabnahme in der Atmosphäre seit dem Höhepunkt der anthropogen verursachten Tritiumkonzentrationen auf der Nordhemisphäre im Jahr 1963 und die damit verbundene Verringerung des Kontrasts zur natürlichen Tritiumaktivität (Solomon et al. 1998, Todt \& Katz 2006). Zur Absicherung der Tritiumbefunde wird in einigen Studien das stabile Zerfallsprodukt ${ }^{3}$ Helium $\left({ }^{3} \mathrm{He}\right)$ verwendet, das sich im Grundwasser mit zunehmender Verweilzeit anreichert (Cook \& Solomon 1997). Ebenso wie bei den stabilen Isotopen werden auch radioaktive Isotope, die nicht Bestandteile des Wassermoleküls sind, zur Charakterisierung von Grundwassersystemen genutzt. Ein Beispiel hierfür ist das radioaktive Isotop des Edelgases Krypton $\left({ }^{85} \mathrm{Kr}\right)$, dessen Gehalt in der Atmosphäre sich durch Freisetzungen aus der Atomindustrie in den letzten Jahrzehnten erhöht hat (Solomon 1998). Ähnlich wie Tritium wurde die Aktivität des radioaktiven Chlorisotops ${ }^{36} \mathrm{Cl}$ in der Atmosphäre durch die Atomwaffentests erhöht (Solomon 1998). Neben diesen anorganischen Isotopen werden auch organische Stoffe als Umwelttracer genutzt. So sind die Konzentrationen der Fluorkohlenwasserstoffe (FCKW) $\mathrm{CCl}_{3} \mathrm{~F}$ (CFC-11), $\mathrm{CCl}_{2} \mathrm{~F}_{2}$ (CFC-12), $\mathrm{C}_{2} \mathrm{Cl}_{3} \mathrm{~F}_{3}$ (CFC-113) in der Atmosphäre durch die Anwendung in Kühlmitteln, Spraydosen und einer Vielzahl industrieller Prozessen, beispielsweise als Lösemittel (Darling et al. 2012), bis in die 1990er Jahre angestiegen. Ein weiterer häufig genutzter Umwelttracer ist Schwefelhexafluorid $\left(\mathrm{SF}_{6}\right)$, das als Isoliergas in der Elektrotechnik verwendet wird. Trotz mittlerweile eingeführter Anwendungsbeschränkungen steigen die Konzentrationen von $\mathrm{SF}_{6}$ in der Atmosphäre noch an (IAEA 2006).

Eine Hauptanwendung der Umwelttracer ist die Abschätzung der Hauptverweilzeit auf Grundlage der bekannten Halbwertszeit der radiokativen Isotope, ähnlich den stabilen Isotopen mittels Lumped-Parametermodellen (Maloszewski \& Zuber 1982). Die Ermittlung der Altersstruktur kann zur Abschätzung von Grundwasserneubildungsanteilen (Egboka et al. 1982) oder Anteilen einer jüngeren Wasserkomponente in Karstgrundwasserleitern (Todt \& Katz 2006, Yager et al. 2013) sowie zur Prognose der Aufenthaltsdauer von Schadstoffen (Farlin et al. 2013) genutzt werden. Ii et al. (1996) schätzen auf Basis der Tritiumaktivität Porosität und Speicherkapazität in geklüfteten Pyroklastiten ab. In Kombination mit künstlichen Markierungversuchen verwendet Einsiedl (2005) die Tritiumaktivität zur Abschätzung der nutzbaren Porositätsanteile der feinklüftigen Gesteinsmatrix und der Karströhren. Ein 
Beispiel für die Anwendung der ${ }^{3} \mathrm{H} /{ }^{3} \mathrm{He}-$ Methode ist die Altersbestimmung der Süßwasserlinse unter der Nordseeinsel Spiekeroog (Röper et al. 2012). Die der Summe von ${ }^{3} \mathrm{H}$ - und ${ }^{3} \mathrm{He}-$ Konzentrationen kann auch ohne bekannte Eintragsfunktion zur Abschätzung der Mischungsverhältnisse von Wasserkomponenten unterschiedlichen Alters genutzt werden, wenn der tritiumbürtige He-Anteil bekannt ist (Sültenfuss \& Massmann 2004). Das Verhältnis der Kryptonisotope ${ }^{85} \mathrm{Kr} / \mathrm{Kr}$, die zur Bestimmung von Verweilzeiten zwischen rund 5 und 50 Jahren genutzt werden können, ist nicht temperaturabhänig und wird durch Ausgasung nicht verändert (Delbart et al. 2014). Bei Annahme einer gleichmäßigen Verteilung der FCKW und von $\mathrm{SF}_{6}$ in der Atmosphäre kann von einer bekannten Eintragsfunktion ausgegangen werden und damit, vergleichbar mit den Isotopentracern, das Alter beziehungsweise Vorhandensein jüngerer Wasserkomponenten ( $<60$ Jahre) abgeschätzt werden (Long \& Puntnam 2009, Delbart 2014, Darling et al. 2012). Weiterhin können Fließbedingungen (Piston-Flow, Exponential-Flow) unterschieden sowie Mischungsverhältnisse bestimmt werden (Darling et al. 2012).

Die Anwendung der ${ }^{3} \mathrm{H}$ und der ${ }^{3} \mathrm{H} / \mathrm{He}-$ Methode ist auf Wässer begrenzt, die mindestens eine Komponente enthalten, die nach Beginn der Atomwaffentest gebildet wurde (Sültenfuss \& Massmann 2004). Aufgrund von möglichen Entgasungsverlusten beim Quellaustritt oder bei der Probenahme ist die He-Methode nicht in allen Karstsystemen anwendbar (Delbart et al. 2014). Weitere Einschränkungen liegen auch dann vor, wenn andere Quellen für ${ }^{3} \mathrm{He}$ vorhanden sind, die eine Hintergrundkonzentration verursachen (Sültenfuss \& Massmann 2004). Die Konzentration der FCKW ist bei der Grundwasserneubildung über die HenryKonstante abhängig von der Temperatur. Große Höhendifferenzen (>1.000 m) zwischen dem Gebiet der Grundwasserneubildung und dem Quellaustritt wirken sich ebenfalls auf die Konzentration in der Wasserprobe aus (Darling et al. 2012). Voraussetzung für Altersbestimmungen mit FCKW und $\mathrm{SF}_{6}$ ist ein Gleichgewichtszustand zwischen atmosphärischen Konzentrationen und dem neu gebildeten Grundwasser (Darling et al. 2012). Es wird damit auch vorausgesetzt, dass die Gaskonzentration in der ungesättigten Zone der bekannten atmosphärischen Zusammensetzung entspricht (Plummer et al. 2006), woraus folgt, dass Informationen hinsichtlich der Eigenschaften der ungesättigten Zone zu ermitteln sind. Insbesondere für die Anwendung von $\mathrm{SF}_{6}$ muss geprüft werden, ob ein Luftüberschuss (excess air) vorliegt und ggf. berücksichtigt werden (Darling \& Gooddy 2012). Da die Lösung von Gasen im Wasser temperatur- und druckabhängig ist, müssen für Altersbestimmung die Druck- und Temperaturbedingungen bei der Grundwasserneubildung abgeschätzt oder experimentell bestimmt werden, beispielsweise mittels Edelgastemperaturen (Althaus 2009). Beim Transport im Grundwasser sind FCKW wie andere organische Kohlenwasserstoffver- 
bindungen Attenuationprozessen ausgesetzt (IAEA 2006), deren Quantität nur mit zusätzlichen Untersuchungen abgeschätzt werden kann. Ähnlich wie beim Tritium ist für die FCKW aufgrund weitgehender Anwendungsbeschränkungen langfristig mit einem abnehmenden Trend der Konzentrationen in der Atmosphäre zu rechnen (Darling et al. 2012). Durch Altablagerungen und Mülldeponien werden jedoch punktuell zusätzliche FCKW freigesetzt (Kühn et al. 2009), aus denen Konzentrationen im Grundwasser resultieren, die über der Gleichgewichtskonzentration aus dem atmosphärischen Eintrag liegen. Die Methode scheidet in diesen Fällen für die Altersbestimmung aus (Kralik et al. 2014), ist aber dann für die Detektion des Einflusses solcher punktueller Quellen auf den Grundwasserkörper, (Kühn et al. 2009), die Verfolgung von kleinräumigen Fließwegen, wie Stockwerksverbindungen über den Brunnenausbau sowie für die Detektion kleinerer junger Einträge in ältere Grundwasserkörper geeignet (Darling et al. 2012). Auch für $\mathrm{SF}_{6}$ sind neben der durchschnittlichen atmosphärischen Zusammensetzung zusätzliche Kontaminationsquellen in städtischen Gebieten und auch geogenen Ursprungs ermittelt worden, so dass lokale Messungen in der Bodenluft sinnvoll erscheinen, wenn $\mathrm{SF}_{6}$ zur Alterbestimmung eingesetzt werden soll (Santella 2008).

\subsubsection{Zusammenfassung der Einschränkungen etablierter hydrochemischer Methoden}

In Karstsystemen ist die Interpretation von Grundwassergleichenplänen in Kombination mit hydraulischer Parametrisierung zur Bestimmung von Fließwegen, die Abgrenzung von Einzugsgebieten oder die Abschätzung von Volumenströmen aufgrund der intensiven Systemheterogenität nicht ausreichend. Gleiches kann auch für andere komplexe und heterogene Systeme, wie stockwerksgegliederte und tektonisch gestörte Grundwasserleiter sowie Systeme mit stark ausgeprägten präferentiellen Fließwegen postuliert werden. Typische Methoden zur Charakterisierung von Karstsystemen zielen auf Untersuchungen am Quellabfluss ab, da dieser Informationen des gesamten Einzugsgebiets integriert. Durch die heute häufig mit mathematischen Modellen unterstützte Zerlegung des integralen Signals in seine Einzelkomponenten auf Basis physikochemischer Parameter oder chemischer Analysen gelöster Hauptionen, natürlicher Isotope, Umwelttracer oder auch REY sind Rückschlüsse auf Verweilzeiten, Mischungsverhältnisse, Größe des Einzugsgebiets und Volumenanteile der unterschiedlichen Porositäten möglich.

Sowohl die Anwendung stabiler Isotope als auch die von Umwelttracern zielt im Wesentlichen auf die Identifizierung der Altersstruktur des Wassers oder unterschiedlicher Bildungs- 
bedingungen ab. Nur im Hochgebirge können stabile Isotope über die Höhenbeziehung auch zur Abgrenzung von Quelleinzugsgebieten genutzt werden. Grundwasserfließbewegungen lassen sich indirekt über die Fließzeit oder die Differenzierung der Herkunft ableiten, womit jedoch keine diskreten Fließwege detektiert werden. Damit ist die Kartierung der Einzugsgebietsgrenze immer noch abhängig von zusätzlichen Informationen zur geologischen Situation, so dass bei deutlichen Kontrasten im Grundwasserchemismus einzelne Gesteinsformationen zum Einzugsgebiet hinzugezählt oder ausgeschlossen werden können. Es bedarf also einer ergänzenden flächenhaften Information.

Methoden, die auf chemischen Analysen beruhen, benötigen einen hinreichenden Kontrast im Chemismus. Dieser kann durch unterschiedliches Gestein im Einzugsgebiet, jahreszeitliche beziehungsweise periodische Schwankungen von Isotopenverhältnissen oder durch unterschiedliche Verweilzeit, die sich im Falle radioaktiver Umwelttracer durch Konzentrationsabnahme des radioaktiven Elements beziehungsweise in der Anreicherung von stabilen Zerfallsprodukten zeigt, zustande kommen. Fehlt dieser Kontrast, ist die jeweilige Methode nur begrenzt oder nicht anwendbar.

Ursache für einen fehlenden oder zu geringen Kontrast sind bei den stabilen Isotopen eine $\mathrm{zu}$ geringe Variabilität der Bildungsbedingungen und bei den anderen vorgestellten Methoden Hintergrundkonzentrationen, die sowohl eine natürliche als auch eine anthropogene Ursache haben können. Im Fall der gelösten Hauptionen und der REY ist sogar beides möglich.

Versuche mit künstlichen Markierungsstoffen stellen den notwendigen Kontrast für eine definierte Punk-zu-Punkt-Beziehung her. Sie sind jedoch auf kurze Versuchszeiten begrenzt und zielen daher auf die schnell fließende Komponente eines Systems ab. Sie werden zur Bestimmung diskreter Fließwege und von Transportparametern eingesetzt, sind aber aufgrund des hohen Aufwands oder der benötigten Vorkenntnisse auf der Stufe einer konzeptionellen Systemcharakterisierung schwer anzuwenden. Auch für einige künstliche Markierungsstoffe gilt, dass ihre Konzentrationen sich sowohl von einer natürlichen oder einer anthropogen Hintergrundkonzentration abheben müssen.

Die Nutzung von REY ist abhängig von einer Differenzierung der Elemente und damit an unterschiedliche Gesteinseigenschaften oder Bildungsbedingungen gebunden. Die Anzahl der zur Verfügung stehenden Stoffe ist mit insgesamt 16 Elementen relativ gering. 
Zeitlich decken künstliche Markierungsversuche und die stabilen Isotope Verweilzeiten bis zu max. einigen Wochen ab. Die Tritiummethode und die Nutzung von FCKW lässt die Abschätzung von Verweilzeiten auf der Zeitskala von Jahren bis Jahrzehnten zu, andere Isostope und Umwelttracer können zur Abschätzung von Verweilzeiten von einigen Jahrzehnten bis zu Jahrtausenden und mehr genutzt werden. Für Verweilzeiten von einigen Monaten bis zu wenigen Jahren sind die vorgestellten Methoden weniger geeignet. Gerade dieser Zeitraum ist neben dem schnell fließenden System für die Bewertung aktueller Schadensereignisse von großer Bedeutung.

Zur Überwindung der aufgezeigten Einschränkungen etablierter hydrochemischer Methoden werden häufig Methodenkombinationen angewendet: Maloszewski et al. (2002) nutzen zur Abschätzung der Volumenanteile und der in Karstsystemen unterschiedlich skalierten Porositäten (Röhren und feinklüfige poröse Matrix) die ${ }^{18} \mathrm{O}-$ Methode in Kombination mit Tritiummessungen zur Abtrennung der schnellen Komponente von der langsamen Komponente. Weitere Beispiele für Verfahrenskombinationen finden sich in vielen der zitierten Fallstudien (beispielsweise Knowles et al. 2010, Long et al. 2008, Tallini et al. 2014) und bei Siebert et al. (2012), einer Studie zur Identifizierung der Herkunft von Mischgrundwässern durch REY-Signaturen in Kombination mit stabilen Iostopen und Tritium, oder bei Doktor et al. (2006), die eine Abschätzung von Wasseranteilen einer Flussversickerung und einer anthropogen beeinflussten Komponente in einem Karstsystem mittels kombinierter Analyse von Hauptionen, stabilen Isotopen und DIC vornehmen.

Die Anwendung solcher Verfahrenskombinationen ist zum einen relativ aufwändig, da unterschiedliche Analysenverfahren zum Einsatz kommen, und trifft zum anderen nicht direkt den Fokus konzeptioneller Studien, in denen es zunächst auf die Abgrenzung von Quelleinzugsgebieten und die qualitative Erfassung von Fließwegen und hydraulischer Zusammenhänge ankommt. Für diese frühen Schritte sind analytische Verfahren gefragt, die mit relativ geringem Aufwand ergänzend zu den etablierten physikalischen Methoden eine erste Systemcharakterisierung erlauben. Die Anwendung von FCKW, die analytisch relativ einfach zu bestimmen sind, bietet hier einen Ansatz, ist jedoch durch die Anzahl unterschiedlicher Stoffe und die Flüchtigkeit der Komponenten limitiert. Weiterhin sind solche Methoden hilfreich, die die Problematik der Hintergrundkonzentration überwinden, die zeitliche Lücke auf der Skala von einigen Monaten schließen und ergänzend zur Auswertung punktueller Quellsignale Informationen zur räumlichen Verteilung hydrogeologischer Eigenschaften liefern können. Außerdem besteht ein Mangel an Methoden, die Rückschlüsse 
auf diskrete Fließwege mit Verweilzeiten von mehr als einigen Wochen und die Lokalisierung von Einzugsgebietsgrenzen zulassen.

Anorganische Stoffe und Isotopen sind in ihrer Anzahl begrenzt, jedoch bietet die Vielzahl organischer Stoffe möglicherweise große Chancen. Viele organische Stoffe sind rein anthropogener Herkunft und müssen sich folglich für eine Nutzung als Indikatoren nicht von einer natürlichen Hintergrundkonzentration abheben. Ihr Nachweis ist damit lediglich durch die Empfindlichkeit der zur Verfügung stehenden Analysenmethoden begrenzt, damit die Grundvoraussetzung für die Ausbildung räumlicher oder zeitlicher Verteilungsmuster erfüllt wird.

\subsection{Nutzung organischer Spurenstoffe zur Charakterisierung von Grundwassersystemen}

\subsubsection{Definition und Eigenschaften organischer Spurenstoffe}

Mehr als 100.000 überwiegend organische Substanzen waren bereits vor der Einführung der EU-Chemikalienverordnung im Jahr 2007 (REACH 2006) von der Europäischen Union im Verzeichnis EINECS der auf dem Markt befindlichen Stoffe registriert (Geiss et al. 1992). Über Freisetzungen aus industriellen Prozessen, das Abwassersystem, Mülldeponien beziehungsweise Altablagerungen sowie landwirtschaftliche Anwendungen gelangt ein Teil von ihnen auch in die aquatische Umwelt (Schwarzenbach et al. 2006).

Als organische Spurenstoffe werden im Sinne dieser Arbeit ausschließlich anthropogen hergestellte organische Verbindungen sowie deren Metabolite und Zerfallsprodukte aus biotischen und abiotischen Prozessen verstanden. Sie bewegen sich in der aquatischen Umwelt in Konzentrationsbereichen unterhalb von $1 \mu \mathrm{g} / \mathrm{l}$, häufig sogar nur von wenigen ng/l (Schwarzenbach et al. 2006). Sie haben bisher in herkömmlich verwendeten und gesetzlichen Parameterlisten (prioritäre Stoffe der EU-Wasserrahmenrichtlinie (WRRL 2000), Trinkwasserverordnung (TrinkwV 2001), Bundes-Bodenschutz- und Altlastenverordnung (BBodschV 1999), Geringfügigkeitsschwellenwerte (LAWA 2004), Abwasserverordnung (AbwV 2004), Oberflächengewässerverordnung (OgewV (2011)) nur in Einzelfällen Eingang gefunden. In der englischsprachigen Literatur finden sich für diese Stoffe die Begriffe „organic micro-pollutants" beziehungsweise "micropollutants" (Heinz et al. 2009, Morasch et al. 2013, Schwarzenbach et al. 2006), „,emerging pollutants“ (Well et al. 2007, Robles-Molina et al. 2014) oder 
auch „emerging contaminants“ (Schriks et al. 2010), die synonym verwendet werden. In den für diese Dissertation verwendeten englischsprachigen Artikeln wird einheitlich der Begriff „organic micro-pollutants“ (OMP) und in deutschsprachigen Abschnitten der Begriff „organische Spurenstoffe“ verwendet.

Im Rahmen dieser Arbeit wurden insgesamt 54 organische Spurenstoffe mit unterschiedlichen Eigenschaften, Anwendungsgebieten und auch Anwendungszeiträumen untersucht. Eine vollständige Liste der untersuchten Stoffe mit den Anwendungsgebieten befindet sich im Kap. 5. In den Kap. 3-5 werden für die jeweilig betrachteten Stoffe zusätzliche Informationen hinsichtlich der Anwendungsmengen und Zeiträume in die Ergebnisdiskussion einbezogen.

Die Ausbildung des Verteilungsmusters eines Stoffs im Grundwasser hängt von dessen Mobilitätseigenschaften beziehungsweise dessen Attenuationspotential ab. Dieses wird durch das Sorptionsverhalten, die Reaktivität gegenüber biotischen oder abiotischen Abbauprozessen und auch die Volatilität bestimmt. Während sich das Sorptionsverhalten nicht-ionischer Stoffe über experimentell bestimmbare Verteilungskoeffizienten (log $\mathrm{K}_{\mathrm{OW}}, \log \mathrm{K}_{\mathrm{OC}}$ ) abschätzen lässt, ist die Mobilität polarer Stoffe von einer Mehrzahl an Prozessen abhängig und daher schwerer abschätzbar (Schaffer et al. 2012). In beiden Fällen hängt die tatsächliche Mobilität im Untergrund neben den Stoffeigenschaften auch von der Beschaffenheit des Aquifermaterials ab. Die im Rahmen dieser Arbeit untersuchten Stoffe umfassen sowohl nicht-ionische Komponenten als auch positiv und negativ sowie zwittrig geladene Ionen. Abbauprozesse hängen stoffspezifisch von den Redoxbedingungen ab. Auch hier weisen die untersuchten Stoffe unterschiedliche Eigenschaften auf. Die abgeschätzten Mobilitätseigenschaften werden ebenfalls in den Kap. 3-5 für die jeweilig betrachteten Stoffe diskutiert. Alle untersuchten Stoffe gelten als nicht volatil.

\subsubsection{Bisheriger Stand zur Nutzung organischer Spurenstoffe als Transportindikatoren in aquatischen Systemen}

Die Untersuchung dieser Stoffe im Rahmen wissenschaftlicher Studien und behördlicher Untersuchungen beginnt im Wesentlichen in den 1990er bis 2000er Jahren, in denen durch zunehmend empfindlichere Analysenmethoden nachgewiesen werden konnte, dass neben Pflanzenschutzmitteln auch Medikamente und deren Metabolite über Abwassersysteme in die Flußsysteme (Ashton 2004) und auch in das Grundwasser gelangen, wie das Beispiel der Berliner Rieselfelder zeigt, auf denen häusliche Abwässer nach Klärung zur Anreicherung 
des Grundwasserleiters versickert wurden (Massmann et al. 2004, Scheytt et al. 1998). Neben dem Abwassersystem stellt der landwirtschaftliche Einsatz eine der Hauptquellen für die Funde von Pestiziden im Grundwasser dar (Nikolaou 2007). Weitere Quellen sind Altablagerungen und industrielle Anlagen. Damit lassen sich flächenhafte Eintragsfunktionen in das Grundwasser (landwirtschaftlicher Einsatz von Pflanzenschutzmitteln, Versickerung von Abwasser, Uferfiltration) von punktuellen beziehungsweise linienförmigen Eintragsfunktionen (Leckagen aus Kanalsystemen und Industrieanlagen, Altablagerungen) unterscheiden (Schulte-Oehlmann et al. 2007).

Bereits in den 1980er Jahren erkannten Simmleit \& Hermanns (1987) das indikative Potential organischer Spurenstoffe und nutzten Lindan und polyzyklische aromatische Kohlenwasserstoffe (PAK) zur Charakterisierung eines Grundwasserneubildungsereignisses in einem Karstsystem. Trotz dieser Hinweise auf die Möglichkeiten zur Nutzung von organischen Spurenstoffen zur Charakterisierung von Grundwassersystemen befasst sich ein großer Anteil der veröffentlichten Untersuchungen überwiegend mit der Detektion und dem Monitoring der Stoffe. Studien, die das Auftreten und die Verteilung der Stoffe zur Charakterisierung hydrogeologischer Eigenschaften nutzen, sind bislang relativ selten und zielen großenteils auf die Identifizierung und Abschätzung von Abwasserbeimengungen im Grundwasser ab. So wird das Auftreten bestimmter Stoffe, wie beispielsweise Carbamazepin, als Indikator für Abwasserleckagen interpretiert (Clara et al. 2004, Katz \& Griffin 2008, Müller et al. 2012). Wolf et al. (2004) leiten Hinweise auf Kanalleckagen in einem Stadtgebiet aus der Detektion iodierter Kontrastmittel ab. Hillebrand et al. (2012) können den Ereignis bezogenen Anteil von Abwasser in einem Quellabfluss mittels Koffein abschätzen.

Darüberhinausgehende Interpretationen finden sich bei Baran et al. (2008), die Massenbilanzen für Triazine und Isoproturon anhand geschätzter Einsatzmengen erstellen. Sie zeigen, dass neben den Stoffeigenschaften auch die zeitlich variierenden Fließverhältnisse Einfluss auf die Konzentration haben. Doummar et al. 2014 können auf Grundlage des Auftretens von Carbamazepin in einer Karstquelle Rückschlüsse auf die Vulnerabilität ziehen.

Die bisher geschilderten Studien zeigen auf, dass organische Stoffe zur Charakterisierung von Grundwassersystemen nutzbar sind. Da sie in großer Quantität und Qualität produziert und verwendet werden, bietet sich hier eine Chance Stoffe zu finden, die die Einschränkungen anderer Verfahren zur Charakterisierung auch komplexer Grundwasser- oder Karstsysteme überbrücken können. 


\subsection{Literatur}

Åberg, G.: The use of natural strontium isotopes as tracers in environmental studies. Water Air Soil Poll. 79, 309-322 (1994)

AbwV: Abwasserverordnung in der Fassung der Bekanntmachung vom 17. Juni 2004 (BGBl. I S. 1108, 2625), die zuletzt durch Artikel 6 der Verordnung vom 2. Mai 2013 (BGB1. I S. 973) geändert worden ist. (2004)

Althaus, R., Klump, S., Onnis, A., Kipfer, R., Purtschert, R., Stauffer, F., Kinzelbach, W.: Noble gas tracers for characterisation of flow dynamics and origin of groundwater: A case study in Switzerland. J. Hydrol. 370, 64-72 (2009)

Ashjari, J., Raeisi, E.: Influences of anticlinal structure on regional flow, Zagros, Iran. Journal of Cave and Karst Studies 68, 118-129 (2006)

Ashton, D., Hilton, M., Thomas, K.V.: Investigating the environmental transport of human pharmaceuticals to streams in the United Kingdom. Sci. Total Environ. 333, 167-184 (2004)

Babiker, M., Gudmundsson, A.: The effects of dykes and faults on groundwater flow in an arid land: the Red Sea Hills, Sudan. J. Hydrol. 297, 256-273 (2004)

Baker, A. and Lamont-Black, J.: Fluorescence of dissolved organic matter as a natural tracer of ground water. Ground Water 39, 745-750 (2001)

Baker, A., Curry, M.: Fluorescence of leachates from three contrasting landfills. Water Res. 38, 2605-2613 (2004)

Baran, N., Lepiller, M., Mouvet, C.: Agricultural diffuse pollution in a chalk aquifer (Trois Fontaines, France): Influence of pesticide properties and hydrodynamic constraints. J. Hydrol. 358, 56-69 (2008)

Barbieri, M., Boschetti, T., Petitta, M., Tallini, M.: Stable isotope $\left({ }^{2} \mathrm{H},{ }^{18} \mathrm{O}\right.$ and $\left.{ }^{87} \mathrm{Sr} /{ }^{86} \mathrm{Sr}\right)$ and hydrochemistry monitoring for groundwater hydrodynamics analysis in a karst aquifer (Gran Sasso, Central Italy). Appl. Geochem. 20, 2063-2081 (2005)

Bau, M., Dulski, P.: Anthropogenic origin of positive gadolinium anomalies in river waters. Earth Planet. Sc. Lett. 143, 245-255 (1996) 
BBodSchV: Bundes-Bodenschutz- und Altlastenverordnung vom 12. Juli 1999 (BGBl. I S. 1554), die zuletzt durch Artikel 5 Absatz 31 des Gesetzes vom 24. Februar 2012 (BGBl. I S. 212) geändert worden ist. (1999)

Birk, S., Geyer, T., Liedl, R., Sauter, M.: Process-based interpretation of tracer tests in carbonate aquifers. Ground Water 43, 381-388 (2005)

Birk, S., Hergarten, S.: Early recession behaviour of spring hydrographs. J. Hydrol. 387, 24-32 (2010)

Brown, R.H., Konoplyantsev, A.A., Ineson, J., Kovalevsky, V.S. (Eds.): Groundwater studies: An international guide for research and practice. 539 S.; UNESCO, Paris (1975)

Clara, M., Strenn, B., Kreuzinger, N.: Carbamazepine as a possible anthropogenic marker in the aquatic environment: investigations on the behaviour of carbamazepine in wastewater treatment and during groundwater infiltration. Water Res. 38, 947-954 (2004)

Cook, P.G., Böhlke, J.K.: Determining timescales for groundwater flow and solute transport. In: Cook and Herczeg (Eds.) "Environmental tracers in subsurface hydrology”. 529 S.; Springer, New York (2000)

Cook, P.G., Solomon, D.K.: Recent advances in dating young groundwater: chlorofluorocarbons, ${ }^{3} \mathrm{H}^{3} \mathrm{He}$ and ${ }^{85} \mathrm{Kr}$. J. Hydrol. 191, 245-265 (1997)

COST ACTION 620: Vulnerability and risk mapping for the protection of carbonate (karst) aquifers. Final Report. 320 S.; EC, Research DG, Bruxelles (2003)

Craig H.: Isotopic variations in meteoric waters. Science 133, 1702-1703 (1961)

d'Amore, F., Scandiffo, G., Panichi, C.: Some observations on the chemical classification of ground waters. Geothermics 12, 141-148 (1983).

Daly, D., Dassargues, A., Drew, D., Dunne, S., Goldscheider, N., Neale, S., Popescu, I. C., Zwahlen, F.: Main concepts of the «European approach» to karst groundwater vulnerability assessment and mapping. Hydrogeol. J. 10, 340-345 (2002)

Dansgard, W.: Stable isotopes in precipitation. Tellus 16, 436-467 (1964) 
Darling, W.G., Gooddy, D.C., MacDonald, A.M., Morris, B.L.: The practicalities of using CFCs and $\mathrm{SF}_{6}$ for groundwater dating and tracing. Appl. Geochem. 27, 1688-1697 (2012)

Delbart, C., Barbecot, F., Valdes, D., Tognelli, A., Fourre, E., Purtschert, R., Couchoux, L., Jean-Baptist, P.: Investigation of young water inflow in karst aquifers using $\mathrm{SF}_{6}-\mathrm{CFC}-$ ${ }^{3} \mathrm{H} / \mathrm{He}_{-}-{ }^{85} \mathrm{Kr}-{ }^{39} \mathrm{Ar}$ and stable isotope components. Appl. Geochem. DOI 10.1016/j.apgeochem.2014.01.011 (2014)

Dia, A., Gruau, G., Olivié-Lauquet, G., Riou, C., Molénat, J., Curmi, P.: The distribution of rare earth elements in groundwaters: assessing the role of source-rock composition, redox changes and colloidal particles. Geochim. Cosmochim. Acta 64, 4131-4151 (2000)

Dimitriou, E., Zacharias, I.: Groundwater vulnerability and risk mapping in a geologically complex area by using stable isotopes, remote sensing and GIS techniques. Environ. Geol. 51, 309-323 (2006)

Doctor, D.H., Alexander Jr., E.C., Petrič, M., Kogovšek, J., Urban, J., Lojen, S., Stichler, W.: Quantification of karst aquifer discharge components during storm events through endmember mixing analysis using natural chemistry and stable isotopes as tracers. Hydrogeol. J. 14, 1171-1191 (2006)

Doerfliger N., Zwahlen F. : Practical guide, groundwater vulnerability mapping in karstic regions (EPIK). 56 S.; Swiss Agency for the Environment, Forests and Landscape (SAEFL), Bern (1998)

Doummar, J., Geyer, T., Baierl, M., Nödler, K., Licha, T., Sauter, M., Carbamazepine breakthrough as indicator for specific vulnerability of karst springs: application on the Jeita spring, Lebanon. Appl. Geochem. DOI 10.1016/j.apgeochem.2014.06.004 (2014)

Egboka, B.C.E., Cherry, J.A., Farvolden, R.N.: Estimation of the percentage of annual groundwater recharge with bomb tritium using a cumulative mass balance method. Pure Appl. Geophys. 120, 330-348 (1982)

Einsiedl, F.: Flow system dynamics and water storage of a fissured-porous karst aquifer characterized by artificial and environmental tracers. J. Hydrol. 312, 312-321 (2005) 
Emblanch, C., Zuppi, G.M., Mudry, J., Blavoux, B., Batiot, C.: Carbon 13 of TDIC to quantify the role of the unsaturated zone: the example of the Vaucluse karst systems (Southeastern France). J. Hydrol. 279, 262-274 (2003)

Etcheverry D., Vennemann, T.: Isotope im Grundwasser. Methoden zur Anwendung in der hydrogeologischen Praxis. Umwelt-Wissen Nr. 0930. 121 S.; Bundesamt für Umwelt, Bern (2009)

Farlin, J., Gall, T., Bayerle, M., Pittois, D., Braun, C., El Khabba, H., Elsner, M., Maloszewski, P.: Predicting pesticide attenuation in a fractured aquifer using lumped-parameter models. Ground Water 51, 276-285 (2103)

Field, M.S., Pinsky, P.F.: A two-region nonequilibrium model for solute transport in solution conduits in karstic aquifers. Journal of Contaminant Hydrology 44, 329-351 (2000)

Field, M.S.: The QTRACER program for tracer-breakthrough curve analysis for karst and fractured-rock aquifers. 137 S.; Environmental Protection Agency, Washington, DC (1999)

Fiorillo, F.: The recession of spring hydrographs, focused on karst aquifers. Water Resour. Manage. 28, 1781-1805 (2014)

Ford, D.C., Williams, P.: Karst Hydrogeology and Geomorphology. 562 S.; John Wiley \& Sons Ltd., West Sussex (2007)

Furtak H., Langguth, H.R.: Zur hydrochemischen Kennzeichnung von Grundwässern und Grundwassertypen mittels Kennzahlen. Mem. IAH-Congress 1965 VII, 86-96 (1967)

Gat, J.R.: Isotope Hydrology: A Study of the Water Cycle. 200 S.; Imperial College Press, London (2010)

Geiss, F., Del Bino, G., Blech, G., Nørager, O., Orthmann, E., Mosselmans, G., Powell, J., Roy, R., Smyrniotis, T., Town, W.G.: The EINECS inventory of existing chemical substances on the EC market. Toxicol. Environ. Chem. 37, 21-33 (1992)

Geyer, T., Birk, S., Licha, T., Liedl, R., Sauter, M.: Multitracer test approach to characterize reactive transport in karst aquifers. Ground Water 45, 36-45 (2007)

Geyer, T., Birk, S., Liedl, R., Sauter, M.: Quantification of temporal distribution of recharge in karst systems from spring hydrographs. J. Hydrol. 348, 452-463 (2008) 
Geyer, T., Birk, S., Reimann, T., Dörfliger, N., Sauter, M.: Differentiated characterization of karst aquifers: some contributions. Carbonate Evaporite 28, 41-46 (2013)

Goldscheider, N., Klute, M., Sturm, S., Hötzl, H.: The PI method - a GIS-based approach to mapping groundwater vulnerability with special consideration of karst aquifers. $\mathrm{Z}$. angew. Geol. 46, 157-166 (2000)

Goldscheider, N., Neukum, C.: Fold and fault control on the drainage pattern of a doublekarst-aquifer System, Winterstaude, Austrian Alps. Acta Carsologica 39, 173-186 (2010)

Goldscheider, N.: Fold structure and underground drainage pattern in the alpine karst system Hochifen-Gottesacker. Eclogae Geol. Helv. 98, 1-17 (2005)

Goldscheider, N.: Tracer tests in karst hydrogeology and speleology. International Journal of Speleology, 37, 27-40 (2008)

Gonfiantini, R., Fröhlich, K., Araguás-Araguás, L., Rozanski, K.: Isotopes in groundwater hydrology. In Kendall, C., McDonnell, J.J. (Eds.): Isotope tracers in catchment hydrology. 839 S.; Elsevier Science B.V., Amsterdam (1998)

Grawunder, A., Merten, D., Büchel, G.: Origin of middle rare earth element enrichment in acid mine drainage-impacted areas. Environ. Sci. Pollut. Res. 21, 6812-6823 (2014)

Han, G., Liu, C.-Q.: Dissolved rare earth elements in river waters draining karst terrains in Guizhou Province, China. Aquat. Geochem. 13, 95-107 (2007)

Hanson, G. N.: Rare earth elements in petrogenetic studies of igneous systems. Annu. Rev. Earth Pl. Sc. 8, 371-406 (1980)

Heinz, B., Birk, S., Liedl, R., Geyer, T., Straub, K.L., Andresen, J., Bester, K., Kappler, A.: Water quality deterioration at a karst spring (Gallusquelle, Germany) due to combined sewer overflow: evidence of bacterial and micro-pollutant contamination. Environ. Geol. 57, 797-808 (2009)

Herold, T., Jordan, P., Zwahlen, F.: The influence of tectonic structures on karst flow patterns in karstified limestones and aquitards in the Jura Mountains, Switzerland. Eclogae Geol. Helv. 93, 349-362 (2000) 
Hillebrand, O., Nödler, K., Licha, T., Sauter, M., Geyer, T.: Caffeine as an indicator for the quantification of untreated wastewater in karst systems. Water Res. 46, 395-402 (2012)

Hölting, B., Coldewey, W.G.: Einführung in die Allgemeine und Angewandte Hydrogeologie. 438 S.; Springer, Berlin Heidelberg (2013)

Hölting, B., Haertlé, T., Hohberger, K.-H., Nachtigall, K. H., Villinger, E., Weinzierl, W., Wrobel, J.-P.: Konzept zur Ermittlung der Schutzfunktion des Bodens. Geol. Jb. C63, 5-24 (1995)

Hölting. B., Matthess, G.: Ein Sporentriftversuch im Zechstein von Korbach/ Hessen. Notizbl. HlfB 91, 237-255 (1963)

Hooker, P.J., O'Nions, R.K., Pankhurst, R.J: Determination of rare-earth elements in USGS standard rocks by mixed-solvent ion exchange and mass-spectrometric isotope dilution, Chem. Geol. 16, 189-196 (1975)

IAEA (International Atomic Energy Agency): Stable isotope hydrology: Deuterium and Oxygen-18 in the water cycle. 337 S.; Technical reports series 210, Internationale Atomenergiebehörde, Wien (1981)

IAEA (International Atomic Energy Agency): Use of chlorofluorocarbons in hydrology: A guidebook, STI/PUB/1238. http://pub.iaea.org/MTCD/publications/PDF/Pub1238_web.pdf (2006)

Ii, H., Ohtsuka, Y., Mori, N., Inagaki, T., Misawa, S., Effective porosity and specific yield of a sedimentary rock determined by a field tracing test using tritium as tracer. Environ. Geol. 27, 170-177 (1996)

Jahnke, C.: Ein neues Klassifikationssystem für Grundwässer und seine Anwendung in känozoischen Porengrundwasserleitern, Grundwasser 2, 62-72 (1999)

Janssen, R.P.T., Verweij, W.: Geochemistry of some rare earth elements in groundwater, Vierlingsbeek, The Netherlands. Water Res. 37, 1320-1350 (2003)

Johannesson, K.H., Stetzenbach, K.J., Hodge, V.F., Kreamer, D.K., Zhou, X.: Delineation of ground-water flow systems in the Southern Great Basin using aqueous rare earth element distributions. Ground Water 35, 805-819 (1997) 
Käss, W., Behrens, H., Matthess, G.: Geohydrologische Markierungstechnik. 557 S.; Borntraeger, Berlin Stuttgart (2004)

Katz, B.G., Chelette, A.R., Pratt, T.R.: Use of chemical and isotopic tracers to assess nitrate contamination and groundwater age, Woodville Karst Plain, USA. J. Hydrol. 289, 36-61 (2004)

Katz, B.G., Griffin, D.W.: Using chemical and microbiological indicators to track the impacts from the land application of treated municipal wastewater and other sources on groundwater quality in a karstic springs basin. Environ. Geol. 55, 801-821 (2008)

Kendall, C., McDonnell, J.J. (Eds.): Isotope Tracers in Catchment Hydrology. 839 S.; Elsevier Science B.V., Amsterdam (1998)

Kiraly, L.: Karstification and Groundwater Flow. Speleogenesis and Evolution of Karst Aquifers 1(3) (2003)

Knowles jr., L., Katz, B.G., Toth, D.J.: Using multiple chemical indicators to characterize and determine the age of groundwater from selected vents of the Silver Springs Group, central Florida, USA. Hydrogeol. J. 18, 1825-1838 (2010)

Kovács, A., Perrochet, P., Király, L., Jeannin, P.-Y.: A quantitative method for the characterisation of karst aquifers based on spring hydrograph analysis. J. Hydrol. 303, 152-164 (2005)

Kovács, A., Perrochet, P.: A quantitative approach to spring hydrograph decomposition. J. Hydrol. 352, 16-29 (2008)

Kralik, M., Humer, F., Fank, J., Harum, T., Klammler, G., Gooddy, D., Sültenfuß, J., Gerber, C., Purtschert, R.: Using ${ }^{18} \mathrm{O} /{ }^{2} \mathrm{H},{ }^{3} \mathrm{H} /{ }^{3} \mathrm{He},{ }^{85} \mathrm{Kr}$ and CFCs to determine mean residence times and water origin in the Grazer and Leibnitzer Feld groundwater bodies (Austria). Appl. Geochem. DOI 10.1016/j.apgeochem.2014.04.001 (2014)

Kühn, S., Kerndorff, H., Oster, H., Struppe, T.: FCKW als Tracer für die Ausbreitung von Altablagerungsemissionen im Grundwasser. Grundwasser 14, 113-125 (2009)

Kulaksız, S., Bau, M.: Anthropogenic dissolvedand colloid/nanoparticle-bound samarium, lanthanum and gadolinium in the Rhine River and the impending destruction of the natural rare earth element distribution in rivers. Earth Planet. Sc. Lett. 362, 43-50 (2013) 
Larocque, M., Cook, P. G., Haaken, K., Simmons, C. T.: Estimating Flow Using Tracers and Hydraulics in Synthetic Heterogeneous Aquifers. Ground Water 47, 786-796 (2009).

LAWA: Länderarbeitsgemeinschaft Wasser: Ableitung von Geringfügigkeitsschwellen für das Grundwasser. 33 S.; LAWA, Düsseldorf (2004)

Liedl, R., Sauter, M., Hückinghaus, D., Clemens. T., Teutsch, G.: Simulation of the development of karst aquifers using a coupled continuum pipe flow model. Water Resour. Res. 39, 1057, DOI:10.1029/2001WR001206 (2003)

Long, A.J., Puntnam, L.D.: Age-distribution estimation for karst groundwater: Issues of parameterization and complexity in inverse modeling by convolution. J. Hydrol. 376, 579-588 (2009)

Long, A.J., Puntnam, L.D.: Linear model describing three components of flow in karst aquifers using ${ }^{18} \mathrm{O}$ data. J. Hydrol. 296, 254-270 (2004)

Long, A.J., Sawyer, F., Puntnam, L.D.: Environmental tracers as indicators of karst conduits in groundwater in South Dakota, USA. Hydrogeol. J. 16, 263-280 (2008)

Loosli, H.H.: A dating method with ${ }^{39}$ Ar. Earth Planet. Sci. Lett. 63, 51-62 (1983)

Mahler, B.J., Garner, B.D.: Using nitrate to quantify quick flow in a karst aquifer. Ground Water 47, 350-360 (2009)

Maillet, E.: Mécanique et physique du globe. Essais d'hydraulique souterraine et fluviale. 218 S.; Hermann, Paris (1905)

Maloszewski, P., Stichler, W., Zuber, A., Rank, D.: Identifying the flow systems in a karsticfissured-porous aquifer, the Schneealpe, Austria, by modelling of environmental ${ }^{18} \mathrm{O}$ and ${ }^{3} \mathrm{H}$ isotopes. J. Hydrol. 256, 48-59 (2002)

Maloszewski, P., Zuber, A.: Determining the turnover time of groundwater systems with the aid of environmental tracers. J. Hydrol. 57, 207-231 (1982)

Maloszewski, P., Zuber, A.: Lumped parameter models for the interpretation of environmental tracer data. Manual on mathematical models in isotope hydrology. IAEA-TECDOC-910: 9-58 (1996) 
Maréchal, J.-C., Ladouche, B., Dörfliger, N., Lachassagne, P.: Interpretation of pumping tests in a mixed flow karst system. Water Resour. Res. 44, W05401, DOI 10.1029/2007WR006288 (2008)

Massmann, G., Knappe, A., Richter, D., Pekdeger, A.: Investigating the Influence of Treated Sewage on Groundwater and Surface Water Using Wastewater Indicators in Berlin, Germany. Acta hydrochim. hydrobiol. 32, 336-350 (2004)

Morasch, B.: Occurrence and dynamics of micropollutants in a karst aquifer, Environ. Pollut. 173, 133-137 (2013)

Müller, B., Scheytt, T., Asbrand, M., Mross de Casas, A.: Pharmaceuticals as indictors of sewage-influenced groundwater. Hydrogeol. J. 20, 1117-1129 (2012)

Murgulet, D., Tick, G.R.: Characterization of flow dynamics and vulnerability in a coastal aquifer system. Ground Water 51, 893-903 (2013)

Neukum, C.: Eine Übersicht zu Methoden und Anwendungen der Validierung von Vulnerabilitätsbewertungen. Grundwasser 18, 15-24 (2013)

Nikolaou, A., Meric, S., Fatta, D.: Occurrence patterns of pharmaceuticals in water and wastewater environments. Anal. Bioanal. Chem. 387, 1225-1234 (2007)

OgewV: Oberflächengewässerverordnung vom 20. Juli 2011 (BGB1. I S. 1429). (2011)

Ozyurt, N.N., Lutz, H.O., Hunjak, T., Mance, D., Roller-Lutz, Z.: Characterization of the Gacka River basin karst aquifer (Croatia): Hydrochemistry, stable isotopes and tritiumbased mean residence times. Sci. Tot. Environ. 487, 173-186 (2014)

Paces, J.B., Wurster, F.C.: Natural uranium and strontium isotope tracers of water sources and surface water-groundwater interactions in arid wetlands - Pahranagat Valley, Nevada, USA. J. Hydrol. 517, 213-225 (2014)

Peng, T.-R., Lu, W.-C., Chen, K.-Y., Zhan, W.-J., Liu, T.-K.: Groundwater-recharge connectivity between a hills-and-plains' area of western Taiwan using water isotopes and electrical conductivity. J. Hydrol. 517, 226-235 (2014) 
Pinault, J.-L., Plagnes, V., Aquilina, L.: Inverse Modeling of the hydrological and the hydrochemical behavior of hydrosystems: Characterization of karst system functioning. Water Res. Res. 37, 2191-2204 (2001)

Piper, A.M.: A graphic procedure in the geochemical interpretation of water analysis.- Trans. Am. Geophys. Union 25, 914-928 (1944)

Plummer, L.N., Busenberg, E., Cook, P.G.: Principles of chlorofluorocarbon dating. In: Use of chlorofluorocarbons in hydrology - A guidebook. 291 S.; IAEA, Wien (2006)

Pronk, M., Goldscheider, N., Zopfi, J., Zwahlen, F.: Percolation and particle transport in the unsaturated zone of a karst aquifer. Ground Water 47, 361-369 (2009)

REACH: Verordnung (EG) Nr. 1907/2006 (2006)

Richter, W., Lillich, W.: Abriss der Hydrogeologie. 281 S.; Schweizerbart, Stuttgart (1975)

Robles-Molina, J., Lara-Ortega, F.J., Gilbert-López, B.,García-Reyes, J.F., Molina-Díaz, A.: Multi-residue method for the determination of over 400 priority and emerging pollutants in water and wastewater by solid-phase extraction and liquid chromatography-time-offlight mass spectrometry, J. Chromatogr. A 1350, 30-43 (2014)

Röper, T., Kröger, K.F., Meyer, H., Sültenfuss, J., Greskowiak, J., Massmann, G.: Groundwater ages, recharge conditions and hydrochemical evolution of a barrier island freshwater lens (Spiekeroog, Northern Germany). J. Hydrol. 454-455, 173-186 (2012)

Rozanski K., Gonfiantini R., Araguas-Araguas L.: Tritium in the global atmosphere: Distribution patterns and recent trends. J. Phys. G: Nucl. Part. Phys. 17, 523-536 (1991)

Sack, A.L., Sharma, S.: A multi-isotope approach for understanding sources of water, carbon and sulfur in natural springs of the Central Appalachian region. Environ. Earth Sci. 71, 4715-4724 (2014)

Santella, N., Ho, D.T., Schlosser, P., Stute, M.: Widespread elevated atmospheric $\mathrm{SF}_{6}$ mixing ratios in the Northeastern United States: Implications for groundwater dating. J. Hydrol. 349, 139-146 (2008) 
Sauter, M.: Quantification and forecasting of regional groundwater flow and transport in a karst aquifer (Gallusquelle, Malm, SW. Germany). 150 S.; Tübinger Geowissenschaftliche Arbeiten C13, Tübingen (1992)

Scanlon, B.R.: Relationships between groundwater contamination and major-ion chemistry in a karst aquifer. J. Hydrol. 119, 271-291 (1990)

Schaffer, M., Boxberger, N., Börnick, H., Licha, T., Worch, E.: Sorption influenced transport of ionizable pharmaceuticals onto a natural sandy aquifer sediment at different $\mathrm{pH}$. Chemosphere $87,513-520$ (2012)

Scheytt, T., Grams, S. Fell, H.: Vorkommen und Verhalten eines Arzneimittels (Clofibrinsäure) im Grundwasser. Grundwasser 2, 67-77 (1998)

Schoeller, H.: Geochimie des eaux souterraines. Revue Inst. Fr. Petrole. 10, 181-213 (1955)

Schriks, M., Heringa, M.B., van der Kooi, M.M.E., de Voogt, P., van Wezel, A.P.: Toxicological relevance of emerging contaminants for drinking water quality. Water Res. 44, 461-476 (2010)

Schulte-Oehlmann, U., Oehlmann, J., Püttmann, W.: Humanpharmakawirkstoffe in der Umwelt: Einträge, Vorkommen und der Versuch einer Bestandsaufnahme. UWSF - Z. Umweltchem. Ökotox. 19, 168-179 (2007)

Schwarzenbach, R.P., Escher, B.I., Fenner, K., Hofstetter, T.B., Johnson, C.A., von Gunten, U., Wehrli, B.: The challenge of micropollutants in aquatic systems. Science 313, 1072-1077 (2006)

Shouyang, H., Lijun, Z., Ruidong, Y., Zheng, S., Xiaohong, Y.: The geochemical characteristics of aqueous rare-earth elements in shallow karst groundwater in Guiyang City, China. Chin. J. Geochem. 30, 114-124 (2011)

Sidle, W.C.: Environmental isotopes for resolution of hydrology problems. Environ. Monit. Assess. 52, 389-410 (1998)

Siebert, C., Rosenthal, E., Möller, P., Rödiger, T., Meiler, M.: The hydrochemical identification of groundwater flowing to the Bet She'an-Harod multiaquifer system (Lower Jordan 
Valley) by rare earth elements, yttrium, stable isotopes $(\mathrm{H}, \mathrm{O})$ and tritium. Appl.

Geochem. 27, 703-714 (2012)

Simmleit, N., Hermann, R.: The behavior of hydrophobic organic micropollutants in different karst water systems I. Transport of micropollutants and contaminant balances during the melting of snow. Water Air Soil Poll. 34, 79-95 (1987)

Smedley, P.L.: The geochemistry of rare earth elements in groundwater from the Carnmenellis area, southwest England. Geochim. Cosmochim. Acta 55, 2167-2779 (1991)

Solomon, D.K., Cook, P.G., Sanford, W.E.: Dissolved gases in subsurface hydrology. In: Kendall, C., McDonnell, J.J. (Eds.): Isotope tracers in catchment hydrology. 839 S.; Elsevier Science B.V., Amsterdam (1998)

Stichler, W., Hermann, A.: Application of environmental isotope techniques in water balance studies of small basins. New Approaches in Water Balance Computations. IAHS Publication 184, 93-112 (1983)

Stueber, A.M., Criss, R.E.: Origin and transport of dissolved chemicals in a karst watershed, southwest Illinois. JAWRA 41, 67-290 (2005)

Stumpp, C., Klaus, J., Stichler, W.: Analysis of long-term stable isotopic composition in German precipitation. J. Hydrol. 517, 351-361 (2014)

Sueker, J.K.: Isotope applications in environmental investigations part II: Groundwater age dating and recharge processes, and provenance of sulfur and methane. Remediation J. 13, 71-90 (2003)

Suksi, J., Rasilainen, K., Pitkänen, P.: Variations in ${ }^{234} \mathrm{U} /{ }^{238} \mathrm{U}$ activity ratios in groundwater-A key to flow system characterisation? Phys. Chem. Earth 31, 556-571 (2006)

Sültenfuß, J., Massmann, G.: Datierung mit der ${ }^{3} \mathrm{He}$-Tritium-Methode am Beispiel der Uferfiltration im Oderbruch. Grundwasser 9, 221-234 (2004)

Sun, J., Tang, C., Wu, P., Strosnider, W. H.J.: Hydrogen and oxygen isotopic composition of karst waters with and without acid mine drainage: Impacts at a SW China coalfield. Sci. Total Environ. 487, 123-129 (2014) 
Tallini, M., Falcone, R.A., Carucci, V., Falgiani, A., Parisse, B., Petitta, M.: Isotope hydrology and geochemical modeling: new insights into the recharge processes and water-rock interactions of a fissured carbonate aquifer (Gran Sasso, central Italy). Environ. Earth Sci. DOI 10.1007/s12665-014-3364-9 (2014)

Taylor, C.J., Greene, E.A.: Hydrogeologic characterization and methods used in the investigation of karst hydrology. In: Rosenberry, D.O., and LaBaugh, J.W.: Field techniques for estimating water fluxes between surface water and ground water. 128 S.; U.S. Geological Survey, U.S. Department of the Interior, Reston (2008)

Taylor, S. R., McLennan, S. H.: The continental crust: Its composition and evolution. 312+XV S.; Blackwell Scientific Publications, Oxford (1985)

Terzić, J., Stroj, A., Frangen, T.: Hydrogeological investigation of karst system properties by common use of diverse methods: a case study of Lička Jesenica springs in Dinaric karst of Croatia. Hydrol. Process. 26, 3302-3311 (2012)

Toran, L., Reisch, C.: Using stormwater hysteresis to characterize karst spring discharge. Ground Water 51, 575-587 (2013)

Toth, D.J., Katz, B.G.: Mixing of shallow and deep groundwater as indicated by the chemistry and age of karstic springs. Hydrol. J. 14, 1060-1080 (2006)

TrinkwV: Verordnung über die Qualität von Wasser für den menschlichen Gebrauch (Trinkwasserverordnung - TrinkwV 2001) neugefasst B. v. 02.08.2013 BGBl. I S. 2977; zuletzt geändert durch Artikel 4 G. v. 07.08.2013 BGBl. I S. 3154. (2001)

Tweed, S.O., Weaver, T.R., Cartwright, I., Schaefer, B.: Behavior of rare earth elements in groundwater during flow and mixing in fractured rock aquifers: An example from the Dandenong Ranges, southeast Australia. Chem. Geol. 234, 291-307 (2006)

Wells, M.J., Fono, L.J., Pellegrin, M-.L., Morse, A.: Emerging pollutants. Water Environ. Res. 79, 2192-2209 (2007)

Wolf, L., Held, I., Eiswirth, M., Hötzl, H.: Impact of leaky sewers on groundwater quality. Acta hydrochim. hydrobiol. 32, 361-373 (2004) 
Worthington, S.R.H.: Diagnostic hydrogeologic characteristics of a karst aquifer (Kentucky USA). Hydrogeol. J. 17,1665-1678 (2009)

WRRL: Wasserrahmenrichtlinie (2000/60/EG): Richtlinie 2000/60/EG des Europäischen Parlaments und des Rates vom 23. Oktober 2000 zur Schaffung eines Ordnungsrahmens für Maßnahmen der Gemeinschaft im Bereich der Wasserpolitik (ABl. Nr. L 327 vom 22/12/2000 S. 0001 - 0073). (2000)

Wundt, W.: Die Kleinstwasserführung der Flüsse als Maß für die verfügbaren Wassermengen. In GRAHAM: Die Grundwässer in der Bundesrepublik Deutschland und ihre Nutzung. Forsch. Dtsch. Landeskunde 104, 4-54 (1958)

Yager, R.M., Plummer, L.N., Kauffman, L.J., Doctor, D.H., Nelms, D.L., Schlosser, P.: Comparison of age distributions estimated from environmental tracers by using binary-dilution and numerical models of fractured and folded karst: Shenandoah Valley of Virginia and West Virginia, USA. Hydrogeol. J. 21, 1193-1217 (2013) 



\section{Occurrence and spatial distribution of organic micro-pollutants in a}

complex hydrogeological karst system during low flow and high flow periods, results of a two-year study

Roland Reh ${ }^{\mathrm{a}}$, Tobias Licha ${ }^{\mathrm{a}}$, Tobias Geyer ${ }^{\mathrm{a}, \mathrm{b}}$, Karsten Nödler $^{\mathrm{a}}$, Martin Sauter ${ }^{\mathrm{a}}$

Citation:

Reh R., Licha T., Geyer T., Nödler, K., Sauter M.: Occurrence and spatial distribution of organic micro-pollutants in a complex hydrogeological karst system during low flow and high flow periods, results of a two-year study. Sci. Total Environ. 443, 438-445 (2013)

http://dx.doi.org/10.1016/j.scitotenv.2012.11.005

a'Universität Göttingen, Geowissenschaftliches Zentrum, Angewandte Geologie, Goldschmidtstraße 3, 37077 Göttingen, Deutschland

${ }^{b}$ Landesamt für Geologie, Rohstoffe und Bergbau (LGRB) im Regierungspräsidium Freiburg, Albertstraße 5, 79104 Freiburg im Breisgau, Deutschland 


\section{Abstract}

Fifty-four different organic micro-pollutants (OMPs) including pharmaceuticals, pesticides, corrosion inhibitors and other typical wastewater compounds such as caffeine are repeatedly analyzed in approximately fifty groundwater observation points in a complex faulted and fractured carbonate aquifer system consisting of three spring catchment areas. With the applied HPLC-MS/MS method, achieving method quantification limits (MQL) of $1.8-28 \mathrm{ng} \mathrm{L}^{-1}$, forty-four of the OMPs are detected in groundwater. Regarding the vertical distribution in the aquifer system the highest variety of OMPs occurs in the shallow aquifer.

Most frequently detected compounds are atrazine together with the metabolites of several triazines, desethylatrazine (DEA) and desisopropylatrazine (DIA), the corrosion inhibitors $1 \mathrm{H}$-benzotriazole and tolytriazoles and as pharmaceutical residues the anti-epileptic drug carbamazepine as well as the analgesic drug phenazone. Median OMP concentrations are in the range of $20-40 \mathrm{ng} \mathrm{L}^{-1}$ with occasionally and locally higher concentrations of up to $6000 \mathrm{ng} \mathrm{L}^{-1}$.

Defined combinations of OMPs occur repeatedly in the same observation wells and allow to distinguish different input functions. The comparison of detection frequency with the number of prescribed doses gives information about the specific persistence of pharmaceuticals. The analgesic phenazone exhibits a peculiar high detection frequency, although it is recently not prescribed in significant amounts. The detection of the estrogen antagonist tamoxifen (6-17 $\left.\mathrm{ng} \mathrm{L}^{-1}\right)$ in a groundwater flow system is reported for the first time. 


\subsection{Introduction}

Organic micro-pollutants (OMPs) such as pharmaceuticals and pesticides are widely distributed in the aquatic environment (Ternes 1998, Kolpin et al. 2002; Schwarzenbach et al. 2006). The vast majority of studies on the occurrence and fate of OMPs in the environment focus on surface waters. Studies on groundwater, especially karst and fractured aquifers, are still heavily underrepresented. Groundwater flow and OMP transport in karst and fractured aquifers is mainly affected by the interaction of rapid conduit flow and slow flow within fissured matrix blocks (e.g. White 1969, Atkinson 1977). Therefore, these aquifer systems are very complex regarding flow and transport parameters. Although OMPs are potential and suitable environmental tracers (Clara et al. 2004, Müller et al. 2012) comprehensive and systematic studies on the occurrence and distribution of OMPs taking into account the spatial and/or temporal variation of OMPs in such aquifers are still scarce (Nödler et al. 2012; Estévez et al. 2012). Previous studies mostly target special problems of discrete conduits (Heinz et al. 2009) and are either limited in number and character of OMPs or sampling points (Metcalfe et al. 2011). Study periods mostly represent hours or days and therefore specific discharge situations (Hillebrand et al. 2012). Longterm observations of an extended spectrum of analytes at all available sampling points within a catchment are uncommon so far.

However, as karst and fractured aquifers are very important but vulnerable drinking water resources this gap of knowledge must be bridged.

In this study the occurrence and distribution of 54 different OMPs in a complex and karstified aquifer system are presented. Many of the chosen OMPs have already demonstrated high detection frequencies in the aquatic environment in previous studies. Additionally, substances with limited data on their occurrence and fate in the environment (e.g. pantoprazole, cetirizine, and tamoxifen) were also included. The chosen study area, characterized as well by the influence of an urban area with industrial sites and waste disposals as by surrounding agricultural uses, exhibits a complex system of several karstified carbonate aquifers. Consequently, a high yield of OMPs with different applications was expected, more so than in sparsely populated areas.

The results from four water sampling campaigns during a two year period (2010-2011) are presented. Considering specific information about the land use and the amount applied in 
the catchment (e.g. the prescribed daily dosage of the analyzed pharmaceuticals, analysis of a banned herbicide) the abundance and persistence of the compounds in the investigated area are evaluated.

\subsection{Material and methods}

\subsubsection{Field site}

The investigation area covers approximately $65 \mathrm{~km}^{2}$, with a city with approximately 24,000 inhabitants and its industrial area located in the center, surrounded by agricultural and forested land uses. The urban area is characterized by intensive industrial applications and exhibits several disused waste disposal sites (Fig. 3.1). Therefore, the release of corrosion inhibitors from industrial applications and pharmaceuticals from the waste disposals are expected.

Concurrently, nearly the complete investigation area is located in a water protection zone. Roughly $10 \%$ of the replenished groundwater is abstracted for public water supply and industrial use. The waste water is discharged to a treatment plant $3.5 \mathrm{~km}$ south of the study area.

The investigated aquifer system is developed within an alternating sequence of shale rocks, fractured aquiferous carbonates and sandstones of the Zechstein formations which are exposed to the surface at the eastern rim of the Rhenish massif (Kulick 1997). The stratigraphic classification (Tab. 3.1), based on the stratigraphic table of Germany ("Deutsche Stratigraphische Tabelle") (Menning \& Hendrich 2002) distinguishes seven Zechstein formations (z1-z7). In this study the aquifers of the z3-z7-formations are united as "upper aquifers" while the z1-aquifer is distinguished as "lower aquifer". For the z2-carbonate no wells or sampling points are existent. The fractured carbonate aquifers of the z1- and z3-formation show partial characteristics that are typical for karstification such as ponors and discrete springs. Through north striking tectonic faults the investigation area is divided into two main tectonic blocks (Kulick 1997), Ense-Scholle in the western part and MeineringhausenScholle in the East (Fig. 3.1).

The investigation area is restricted by two main spring areas, which are located in the North and in the South. The total discharge of each spring area, consisting of a number of conti- 
guous single springs, amounts about $75 \mathrm{~L} \mathrm{sec}^{-1}$. East of the fault "Westheimer Abbruch" a number of small springs feed a river with a discharge of $100 \mathrm{~L} \mathrm{sec}^{-1}$. Due to the positions of the main spring areas and the hydraulic head subsurface water divides are expected within the study area (Hölting \& Matthess 1963) leading to preferential north and south directed groundwater flow.

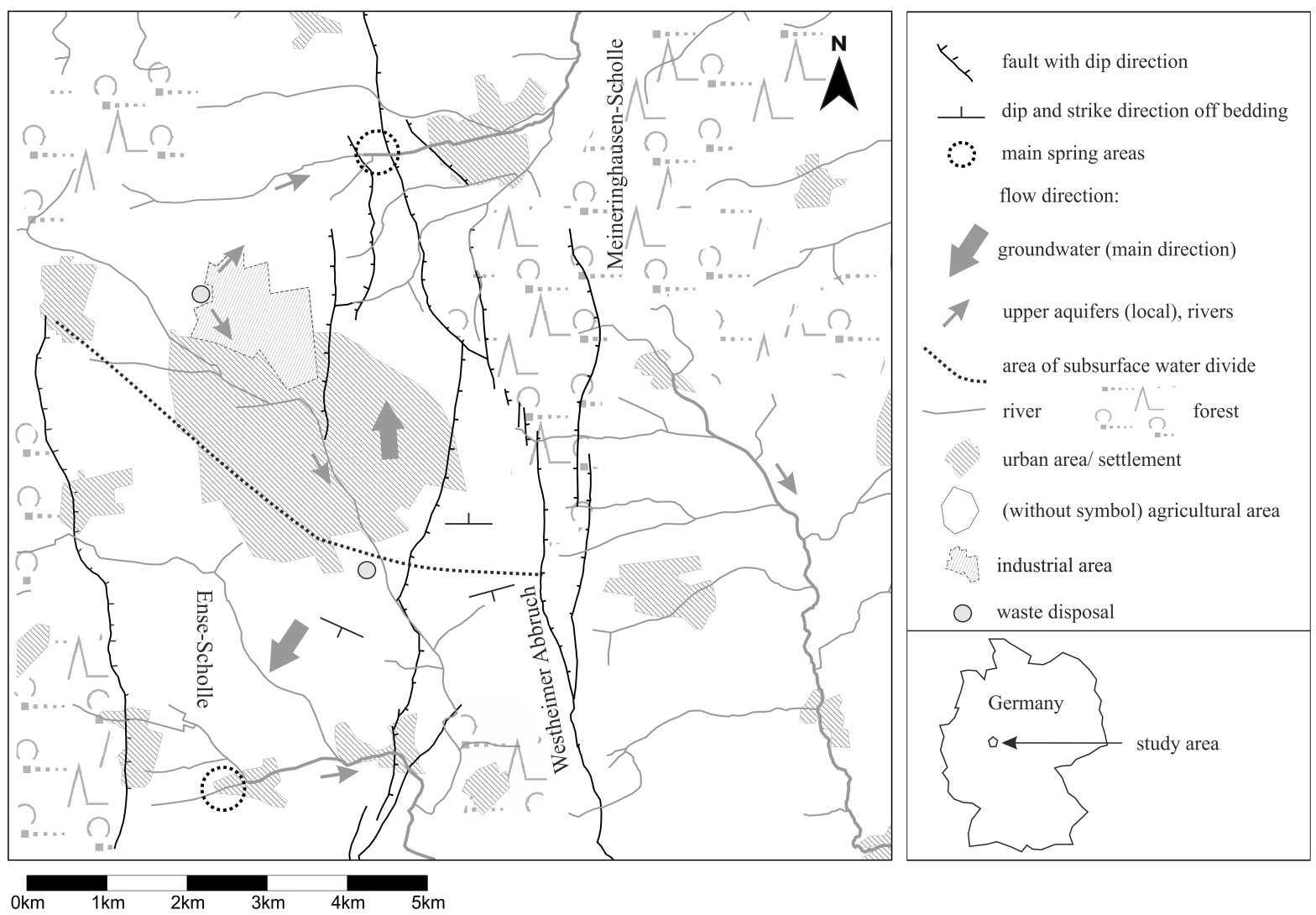

Fig. 3.1: Study area with characterization of land uses, geological structures and estimated groundwater flow directions, water divide according to Hölting \& Matthess (1963) 


\subsubsection{Sampling}

Samples were taken from 51 sampling points consisting of observation and abstraction wells, springs, and rivers. 44 of the sampling points represent groundwater (Fig. 3.2, p. 55). The number of sampling points associated to the different aquifer levels is displayed in Tab. 3.1. Natural springs are correlated to the outlet position of highly permeable subsurface flow pathways (e.g. solution enlarged fractures). Two of the observed springs are located in carboniferous shale west of the investigation area.

Most groundwater wells are established in either the z1- or the z3-aquifer. Additionally some well screens hydraulically connect different aquifer levels. In total, the sampling points represent 46 locations.

Tab. 3.1: Stratigraphic table of the local Zechstein formations based on Menning \& Hendrich (2002) with identification of the aquifers and allocation of sampling points

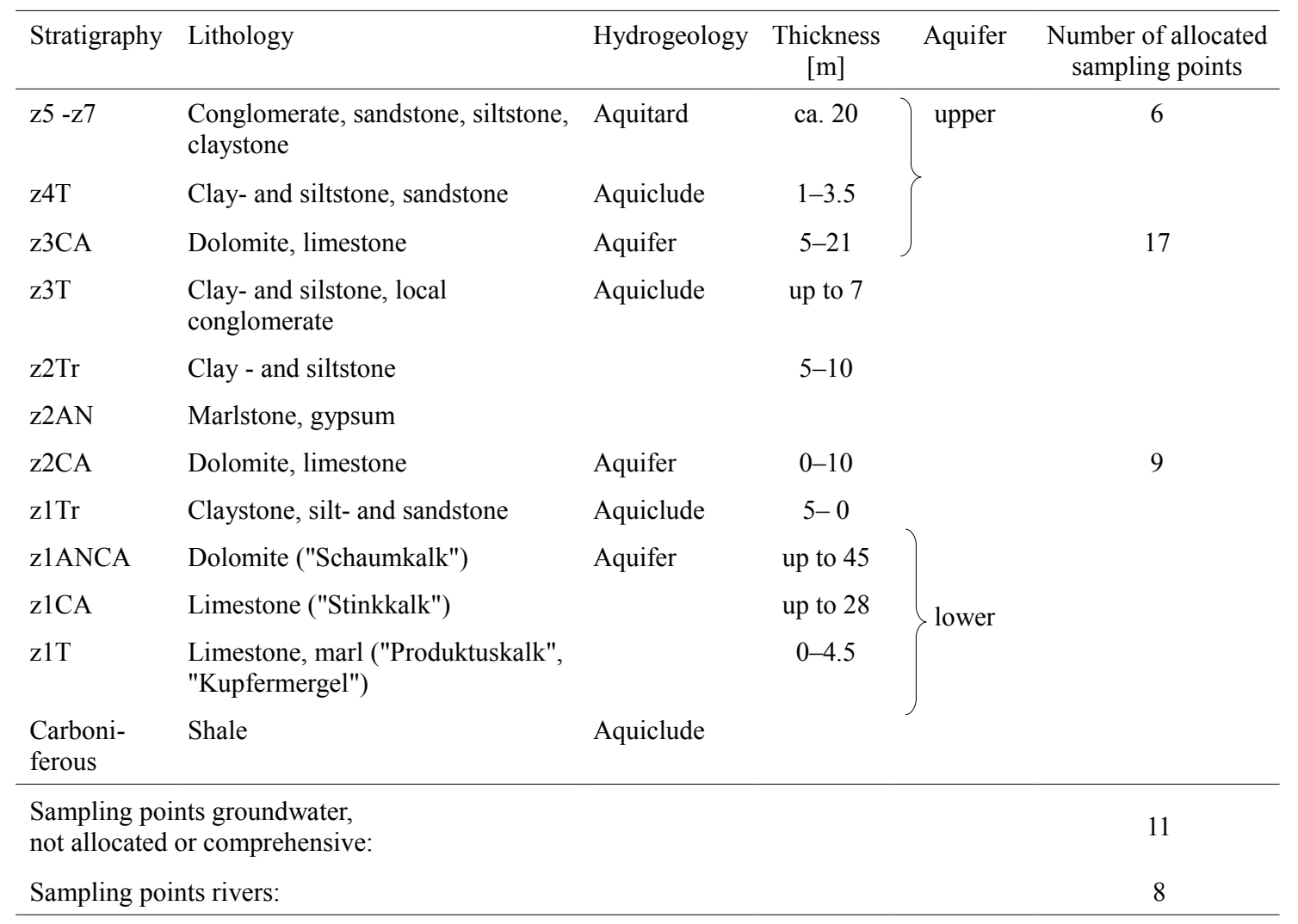


The samples were taken during four sampling campaigns in spring and late summer in 2010 and 2011 representing high and low flow periods respectively. Between spring and late summer groundwater levels decline by $0.2-2.0 \mathrm{~m}$ and the discharge of the main springs decreases by $30-60 \%$. Additional samples were taken 2 weeks after snow melt in January 2011 representing a special recharge event. According to the types of sampling points, different sampling methods were applied. Samples from spring water and from surface water were directly taken as grab samples (500 mL glass bottles, screw cap). Wells for regular groundwater abstraction were sampled directly from the rising main during their continuous production. In observation wells a submerged pump was installed for well purging purposes and the sample was taken immediately afterwards using a PTFE bailer. The samples were transported in $500 \mathrm{~mL}$ glass bottles to the laboratory in a dark, cooled box and extracted within 24 hours after sampling.

\subsubsection{Chemical analysis}

A multi-residue analytical method based on solid phase extraction (SPE) and high-performance liquid chromatographic separation with tandem mass spectrometric detection (HPLCMS/MS) was used for the analysis. Details regarding the methodology and analytes were published previously (Nödler et al. 2010). The method quantification limits (MQL) reported therein are between 1.2 and $28 \mathrm{ng} \mathrm{L}^{-1}$. The analytes cotinine (nicotine metabolite), atenolol acid (metabolite of atenolol and metoprolol), haloperidol (antipsychotic), tamoxifen (estrogen antagonist for breast cancer treatment), terbuthylazine (herbicide), 4-OH-diclofenac (diclofenac metabolite), desamino-SMX, and 4-nitro-SMX (both sulfamethoxazole metabolites, see Nödler et al. 2012) were included in the presented study. The MS/MS parameters of the additional analytes were added to the methodology of Nödler et al. (2010). Further information can be found in Tab.3.2. 
3 Occurrence and spatial distribution of organic micro-pollutants in a complex hydrogeological karst system during low flow and high flow periods, results of a two-year study

Tab. 3.2: Electrospray (ESI)-MS-MS-parameters of the analytes additional to Nödler et al. (2010)

\begin{tabular}{lllllllll}
\hline Compound & Quantifier & $\begin{array}{l}\text { Cap U } \\
{[\mathrm{V}]^{\mathrm{a}}}\end{array}$ & $\begin{array}{l}\text { CE } \\
{[\mathrm{V}]^{\mathrm{b}}}\end{array}$ & Qualifier & $\begin{array}{l}\text { Cap U } \\
{[\mathrm{V}]^{\mathrm{a}}}\end{array}$ & $\begin{array}{l}\text { CE } \\
{[\mathrm{V}]^{\mathrm{b}}}\end{array}$ & $\begin{array}{l}\text { IS }^{\mathrm{c}} \\
\text { MQL }^{\mathrm{d}} \\
{\left[\mathrm{ng} \mathrm{L}^{-1}\right]}\end{array}$ \\
\hline Haloperidol & $376 \rightarrow 165$ & 55 & -18.5 & $376 \rightarrow 123$ & 55 & -30.5 & Atenolol-D $_{7}$ & 4.0 \\
Tamoxifen & $372 \rightarrow 72$ & 62 & -14.5 & $372 \rightarrow 129$ & 62 & -21.0 & Loratadine-D $_{4}$ & 6.0 \\
Cotinine & $177 \rightarrow 80$ & 55 & -15.5 & $177 \rightarrow 98$ & 55 & -11.0 & Atenolol-D $_{7}$ & 4.0 \\
Terbuthylazine & $230 \rightarrow 174$ & 45 & -11.0 & $230 \rightarrow 104$ & 45 & -25.5 & Atrazine-D $_{5}$ & 3.0 \\
Atenolol acid & $268 \rightarrow 191$ & 60 & -12.0 & $268 \rightarrow 145$ & 60 & -17.5 & Atenolol-D $_{7}$ & 4.0 \\
Desamino-SMX & $237 \rightarrow 141$ & -35 & 13.0 & $237 \rightarrow 77$ & -35 & 25.5 & SMX-13 $^{13} \mathrm{C}_{6}$ & 1.5 \\
4-nitro-SMX & $282 \rightarrow 138$ & -40 & 20.5 & $282 \rightarrow 186$ & -40 & 13.0 & SMX- $^{13} \mathrm{C}_{6}$ & 1.0 \\
4-OH-diclofenac & $310 \rightarrow 266$ & -35 & 13.0 & $310 \rightarrow 230$ & -35 & 8.5 & Ibuprofen-D $_{3}$ & 3.0 \\
\hline
\end{tabular}

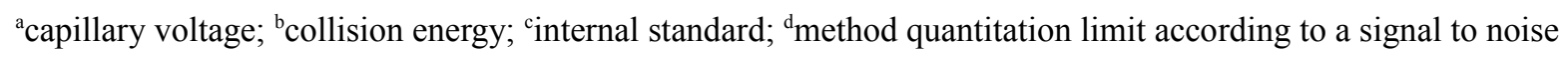
ratio $(\mathrm{S} / \mathrm{N})$ of 10

\subsection{Results and discussion}

\subsubsection{Detection frequency of OMPs in groundwater}

Tab. 3.3 lists the detected compounds and their detection frequencies together with the related applications. In total, 44 compounds were detected in the groundwater within the investigated area representing $81 \%$ of analyzed OMPs. Six further compounds (tetrazepam, cetirizine, sertraline, pantoprazole, atenololic acid and 4-OH-diclofenac) were never found in groundwater although they are detected in rivers within the study area. Naproxen, iopamidol, iopromide, and diazepam were never detected in either surface nor groundwater.

Atrazine and the degradation product of several triazines desethylatrazine (DEA) occur in $61 \%$ and $71 \%$ of 163 analyzed groundwater samples, respectively. They are widespread in the investigation area and were detected in 32 of 44 groundwater sampling points. Hence, the second possible degradation product desisopropylatrazine (DIA) is also common and determined in $23 \%$ of the groundwater samples. The most frequently detected pharmaceuticals are carbamazepine, phenazone, primidone and metoprolol with detection frequencies of 10 $13 \%$. Caffeine, a further frequently detected OMP (detection frequency $9 \%$ ), is a typical indicator for a recent input of untreated wastewater (Hillebrand et al. 2012). The results of this study show in contrast to most of the detected OMPs a temporal variation in its appearance. This may be attributed to its easy attenuation together with a non continuous input. 
Tab. 3.3: Detection frequency of OMPs relating to the number of groundwater samples with minimum(min.), median- and maximum- (max.) concentrations of all groundwater samples

\begin{tabular}{|c|c|c|c|c|c|c|}
\hline Compound & Application & $\begin{array}{c}\text { detection } \\
\text { frequency } \\
{[\%]}\end{array}$ & min.- & $\begin{array}{l}\text { median- } \\
\text { tration }[1\end{array}$ & $\begin{array}{l}\text { max.- } \\
\left.{ }^{-1}\right]\end{array}$ & \multirow{2}{*}{$\begin{array}{c}\begin{array}{c}\text { number of } \\
\text { samples }\end{array} \\
163\end{array}$} \\
\hline Desethylatrazine & Herbicide metabolite & 70.6 & 2.3 & 17.6 & 171.1 & \\
\hline Atrazine & Herbicide & 60.7 & 1.7 & 7.8 & 86.1 & 163 \\
\hline 1H-Benzotriazole & Corrosion inhibitor & 40.5 & 4.9 & 43.4 & 3241.8 & 163 \\
\hline Tolyltriazole & Corrosion inhibitor & 31.3 & 5.2 & 23.4 & 213.4 & 163 \\
\hline Desisopropylatrazine & Herbicide metabolite & 22.7 & 6.0 & 22.1 & 1810.8 & 163 \\
\hline Mecoprop & Herbicide & 17.2 & 1.2 & 107.9 & 2903.5 & 163 \\
\hline Terbuthylazine & Herbicide & 14.8 & 3.2 & 5.4 & 92.7 & 135 \\
\hline Carbamazepine & Anticonvulsant, sedative & 12.9 & 2.4 & 38.4 & 346.7 & 163 \\
\hline Diuron & Herbicid & 12.9 & 3.3 & 5.3 & 20.4 & 163 \\
\hline Phenazone & Analgesic/ anti-inflammatory & 12.3 & 2.5 & 147.0 & 6214.0 & 163 \\
\hline Primidone & Anticonvulsant, sedative & 10.4 & 3.3 & 15.1 & 397.3 & 163 \\
\hline Isoproturon & Herbicide & 10.4 & 3.3 & 6.4 & 78.0 & 163 \\
\hline Metoprolol & Antihypertensive & 10.0 & 4.9 & 16.0 & 73.7 & 140 \\
\hline Caffeine & Stimulant & 8.6 & 5.0 & 9.8 & 102.3 & 163 \\
\hline Citalopram & Antidepressant & 8.0 & 4.7 & 10.1 & 69.4 & 163 \\
\hline Haloperidol & Antipsychotic & 7.4 & 4.1 & 6.5 & 25.8 & 163 \\
\hline Sulfamethoxazole & Antibiotic & 7.4 & 3.2 & 5.4 & 42.2 & 163 \\
\hline Loratadine & Antiallergic & 4.9 & 2.8 & 6.1 & 8.6 & 163 \\
\hline Cotinine & Nicotine metabolite & 4.4 & 6.0 & 27.2 & 320.3 & 135 \\
\hline Iohexol & Iodinated contrast media & 4.3 & 22.4 & 26.2 & 74.9 & 163 \\
\hline Theobromine & Stimulant & 3.7 & 11.9 & 18.8 & 273.4 & 163 \\
\hline Roxithromycin & Antibiotic & 3.7 & 11.2 & 15.4 & 23.2 & 163 \\
\hline Clarithromycin & Antibiotic & 3.7 & 8.7 & 15.3 & 24.5 & 163 \\
\hline Paracetamol & Analgesic/ anti-inflammatory & 3.1 & 24.4 & 3076.4 & $35,000.0$ & 163 \\
\hline Tamoxifen & Cancer treatment & 3.1 & 6.0 & 12.2 & 16.5 & 163 \\
\hline Sotalol & Antihypertensive & 2.9 & 5.9 & 11.8 & 54.3 & 140 \\
\hline Clofibric acid & Lipid regulator & 2.5 & 3136.9 & 3732.1 & 4210.5 & 163 \\
\hline Paraxanthine & Stimulant & 2.5 & 3.6 & 9.4 & 156.4 & 163 \\
\hline Metazachlor & Herbicid & 2.5 & 5.5 & 5.9 & 20.0 & 163 \\
\hline Bezafibrate & Lipid regulator & 1.8 & 9.3 & 27.4 & 68.4 & 163 \\
\hline Erythromycin & Antibiotic & 1.8 & 5.5 & 6.4 & 392.4 & 163 \\
\hline Atenolol & Antihypertensive & 1.8 & 4.7 & 5.2 & 6.2 & 163 \\
\hline Desamino-SMX & Antibiotics, metabolites & 1.1 & 6.0 & 6.0 & 6.0 & 93 \\
\hline 4-Nitro-SMX & Antihypertensive & 1.1 & 4.1 & 4.1 & 4.1 & 93 \\
\hline Diclofenac & Analgesic/ anti-inflammatory & 0.6 & 3.1 & 3.1 & 3.1 & 162 \\
\hline 3-Methylxanthine & Stimulants, metabolites & 0.6 & 611.2 & 611.2 & 611.2 & 163 \\
\hline 1-Methylxanthine & Stimulant, metabolite & 0.6 & 261.4 & 261.4 & 261.4 & 163 \\
\hline Trimethoprim & Antibiotic & 0.6 & 194.8 & 194.8 & 194.8 & 163 \\
\hline Theophylline & Stimulant, metabolite & 0.6 & 79.5 & 79.5 & 79.5 & 163 \\
\hline Iomeprol & Iodinated contrast media & 0.6 & 55.4 & 55.4 & 55.4 & 163 \\
\hline Fluoxetine & Antidepressant & 0.6 & 26.8 & 26.8 & 26.8 & 163 \\
\hline Ibuprofen & Analgesic/ anti-inflammatory & 0.6 & 23.0 & 23.0 & 23.0 & 163 \\
\hline Gemfibrozil & Lipid regulator & 0.6 & 10.3 & 10.3 & 10.3 & 163 \\
\hline Benzoylecgonine & Cocaine metabolite & 0.6 & 2.3 & 2.3 & 2.3 & 163 \\
\hline
\end{tabular}


The potential input mass of pharmaceuticals can be estimated by the "defined daily dose" (DDD), which is defined as the "assumed average maintenance dose per day for a drug used for its main indication in adults" (WHO). The prescribed DDDs in the investigated area for the year 2010 are presented in Tab. 3.4. The data comprise the prescribed doses only for members of the public health insurances. However, this already covers around $85 \%$ of the general public. The number of DDDs is declared as total for the federal state and converted by the authors to the number of inhabitants within the investigation area. The amount of some compounds is underestimated as some drugs are also available without prescription. Although regional deviations in prescribed doses may occur, the used data are representative for about 5 million inhabitants. The prescribed doses can change gradually over years or sudden when a pharmaceutical is replaced. Nevertheless, under or over represented pharmaceuticals relative to the current prescribed amounts allow conclusions with respect to persistency (over represented), attenuation (under represented) or even if the applied amount is changed. Metoprolol is the most frequently prescribed pharmaceutical of the analyzed OMPs, however, regarding the values of DDDs the estimated mass of ibuprofen is twice as much. Ibuprofen is not frequently detected in the investigation area although it is additionally an over-the-counter drug. This may be explained by its high attenuation potential in aquatic environments (Buser et al. 1999). The same may be also valid for theophylline, which is readily biodegradable (Richardson \& Bowron 1985). The excretion rates of unchanged metoprolol (5\%) and carbamazepine (3\%) are in the same order of magnitude (Mc Evoy 2003; Clara et al. 2004). Although the potential input mass of metoprolol is four to five times higher than that of carbamazepine the detection frequency is nearly equal for both compounds thus indicating better attenuation of metoprolol in groundwater. Diclofenac occurs with a low frequency predominantly in river samples. This is surprising as it is an over-the-counter drug and the mass released will be greatly underevaluated from Table 3.4. Its low detection frequency and its absence in groundwater suggest significant attenuation (Buser et al. 1999). In contrast to diclofenac, phenazone exhibits a peculiar high detection frequency, although it is recently not prescribed in significant amounts. The same applies to primidone. Its low potential input together with its relatively high detection frequency of $10 \%$ may suggest a high persistence for this compound in karst and fractured aquifers. Tamoxifen, an estrogen antagonist, is applied for cancer therapy since the 1980's. In groundwater tamoxifen exists as cation ( $\mathrm{pK}_{\mathrm{a}} 8.87$, Hilal et al. 1995). Its occurrence is reported for river systems (Ashton et al. 2004) but to the authors' knowledge not yet for aquifers. In the investigation area tamoxifen occurs preferentially in springs of the upper aquifers north of the urban area and also in one spring south of it. Concentrations between 6 and $17 \mathrm{ng} \mathrm{L}{ }^{-1}$ 
were detected in groundwater samples predominantly in samples from winter campaigns. The occurrence of compounds with a low potential input mass, like tamoxifen, can be interpreted as them being sufficiently persistent to be detected in groundwater.

Tab. 3.4: Number of DDD in the investigation area in 2010, value of DDD and estimated potential input mass

\begin{tabular}{|c|c|c|c|}
\hline Compound & Number of DDD & $\mathrm{DDD}[\mathrm{mg}]$ & $\begin{array}{c}\text { Estimated annual } \\
\text { potential input mass }[\mathrm{kg}]\end{array}$ \\
\hline Ibuprofen** & 105,044 & 1200 & 126 \\
\hline Metoprolol & 459,373 & 150 & 69 \\
\hline Carbamazepine & 14,110 & 1000 & 14 \\
\hline Diclofenac** & 12,4250 & $100-280$ & 12 \\
\hline Theophylline** & 30,573 & 400 & 12 \\
\hline Pantoprazole** & 24,3013 & $20-40$ & 10 \\
\hline Erythromycin & 3528 & $1000-2000$ & 7 \\
\hline Clarithromycin & 4703 & $375-1000$ & 5 \\
\hline Bezafibrate & 6663 & 600 & 4 \\
\hline Atenolol & 24,693 & $75-150$ & 2 \\
\hline Sotalol & 11,759 & 160 & 2 \\
\hline Citalopram & 78,783 & 20 & 2 \\
\hline Primidone & 1176 & 1250 & 1 \\
\hline Roxithromycin & 4312 & $150-300$ & 1 \\
\hline Tetrazepam & 6663 & 125 & 1 \\
\hline Gemfibrozil & 392 & 1200 & $<1$ \\
\hline Fluoxetine & 15,678 & 20 & $<1$ \\
\hline Trimethoprim & 784 & $150-400$ & $<1$ \\
\hline Tamoxifen & 9015 & 20 & $<1$ \\
\hline Haloperidol & 4703 & $3.3-8$ & $<1$ \\
\hline Cetirizine** & 3528 & 10 & $<1$ \\
\hline Loratadine** & 392 & 10 & $<1$ \\
\hline Phenazone & $0^{*}$ & 3000 & \\
\hline
\end{tabular}

$\mathrm{DDD}=$ defined daily dose from WHO Anatomical Therapeutic Chemical (ATC) Classification

Number of DDD converted to investigation area (24,000 inhabitants)

*number of prescriptions $<4$

** available without prescription, therefore data are underestimated

Generally, the urban area and the North of the investigation area exhibit a higher diversity of OMPs whereas east of the main fault, within the Meineringhausen-Scholle, a lower number of OMPs is encountered. In Fig. 3.2 the distribution of detected OMPs per sampling point for all sampling campaigns is displayed. The depiction distinguishes between the upper aquifers $(z 3-z 7)$ and the lower aquifer (z1). A significant number of OMPs ( $>7)$ is detected in the following areas: 
a) Sampling points of the upper aquifer in the urban area. Most of these sampling points are observation wells, associated to former waste disposals.

b) In a spring feed by the upper aquifer and in the main spring area in the North.

c) In an observation well at the southern city boundary, which is located in an area where the z1-aquifer is exposed to the surface. The location is close to a former waste disposal, a former wastewater treatment plant, an existing wastewater conduit, an influent river and a fault zone.

d) In the South a higher diversity of OMPs is exclusive to springs of low discharge. The springs are located close to the features (wastewater conduit, river, fault zone) which cross the aforementioned observation well (see c).

The standard deviation, in this case a dimension for the seasonal variation of the number of OMPs at a sampling point, is commonly low in wells and in the main spring area in the South (0-2). In some springs of the upper aquifers a higher deviation (2-4) is observed indicating seasonal changes or event induced input or dilution. The highest standard deviation is calculated for the river samples (4-7).

OMPs were also detected in the lower aquifer (z1-aquifer) in the Meineringhausen-Scholle even if single well screens in the lower aquifer exclude aquifer connections. Thus, they must have passed confining layers of minimum $100 \mathrm{~m}$ in thickness or must have been transported over long distances $(>2 \mathrm{~km})$ from a location where the elevation of the z1-aquifer is close to the surface. All sampling points in this geological situation share a small number of OMPs. The dominant compounds of the lower aquifer are triazines. 


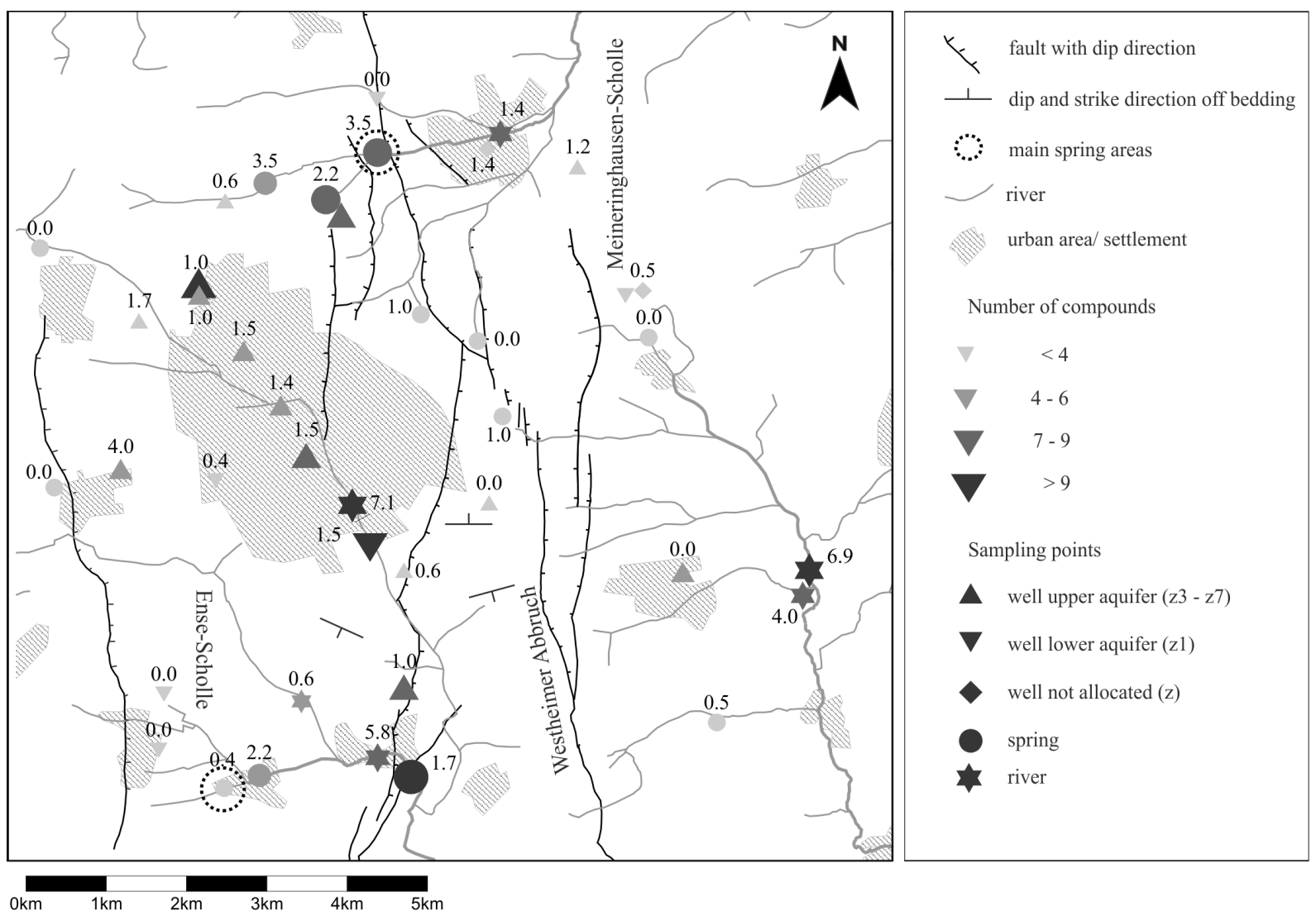

Fig. 3.2: Number of different OMPs per sampling point as median of all sampling campaigns with values of standard deviation.

\subsubsection{Triazines and triazoles}

In Germany atrazine was used as a pesticide until 1992, afterwards its application for agricultural purposes was prohibited. So it is likely that the input of most of the detected atrazine dates back approximately 20 years. Atrazine is degradable in natural environments (Erickson 1989) even though rates are low in aerobic groundwater (Agverted et al. 1992). Significant degradation products are DEA and DIA (Geller 1980). Elevated concentrations of triazines were detected in the springs north of the city (sum triazines max. $160 \mathrm{ng} \mathrm{L}^{-1}$ ) but the highest concentrations were detected within the urban area (max. $2000 \mathrm{ng} \mathrm{L}^{-1}$ ). Elevated concentrations were also detected in a small spring south of the city. Based on this distribution it is not evident, that the input of the triazines is located only in agricultural areas. 
The ratio of atrazine to the degradation products (DEA, DIA) may offer information on its degradation rate (Meyer et al. 2009). The different mass fractions of atrazine, DEA and DIA are plotted as a ternary diagram exemplary for the two sampling campaigns in March and September 2011 (Fig. 3.3). The results of the two sampling campaigns in 2010 are similar. Most of the data exhibit a high fraction of DEA. These data are mostly from sampling points of the agricultural area surrounding the city and represent results of the lower and the upper aquifers. In contrast to this, wells in the urban area show higher proportions of DIA. Comparable triazine fractions were detected in a well which is developed in the z1-aquifer in a silvicultural area northeast of the city.

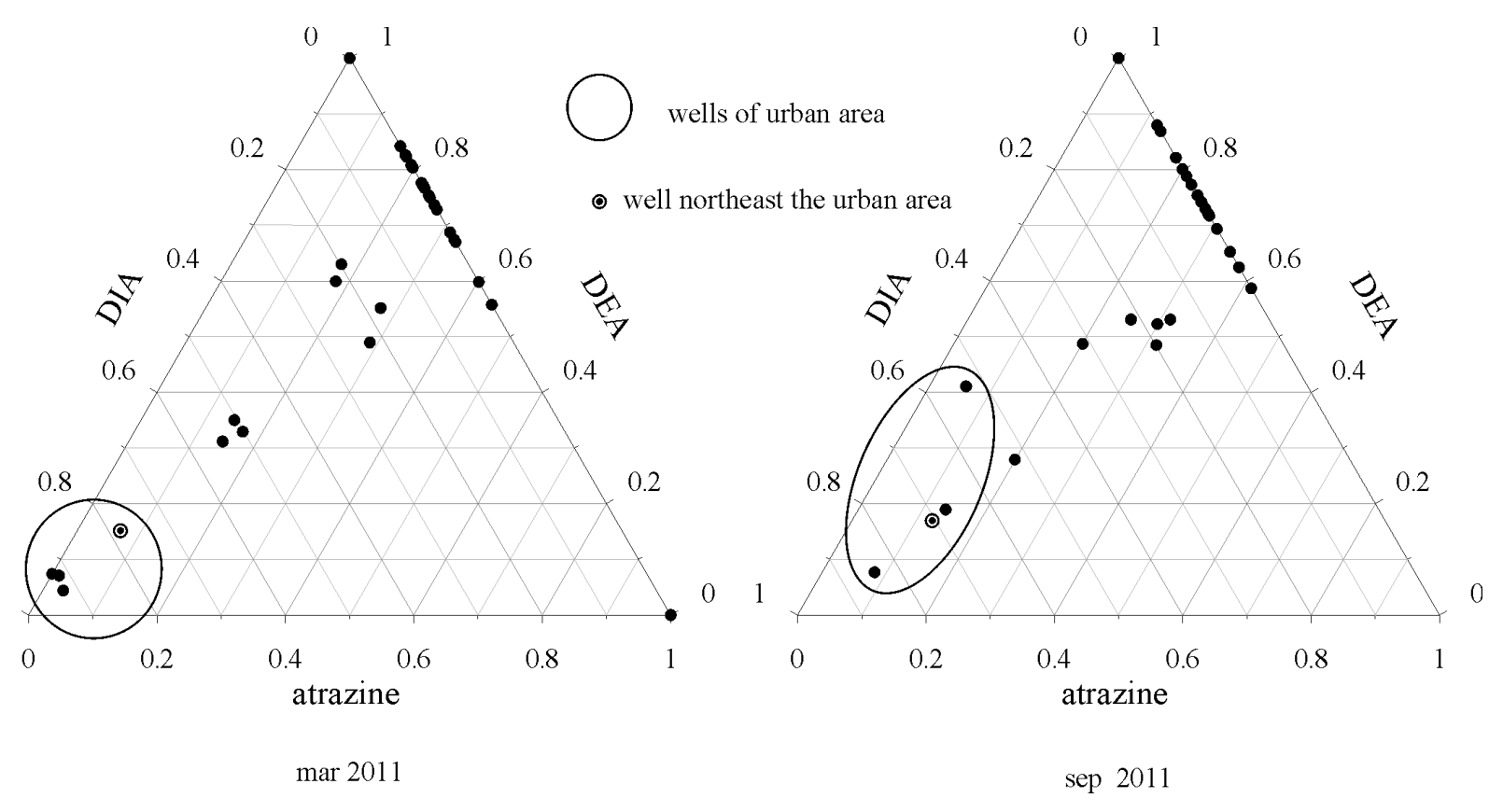

Fig. 3.3: Ternary diagram of triazine mass fractions, data from sampling campaigns March 2011 and September 2011, same results are obtained in March and August 2010

1H-benzotriazole (BT) was detected in $41 \%$ of the groundwater samples at 22 sampling points while tolytriazoles (TT) are found to be less abundant and only occured in $31 \%$ of the samples at 17 sampling points. Due to their applications as corrosion inhibitor their input is located within the urban area. Here BT concentrations reached a maximum of $750 \mathrm{ng} \mathrm{L}^{-1}$ in groundwater. Another area of elevated BT concentrations is located north of the city. The urban area is crossed by a small river, which loses water into the subsurface and exhibits high concentrations of BT. The concentration in the river water was with $6500 \mathrm{ng} \mathrm{L}^{-1}$ much higher than that of the groundwater. In the southwestern part of the Ense-Scholle and within 
the Meineringhausen-Scholle BT was only detected in a low number of sampling points. Exceptions are a river and a well located in a forest. In this well concentrations between 1600 and $3200 \mathrm{ng} \mathrm{L}^{-1}$ were detected. The reason for this relatively high concentration, located in a forested area, is not yet clear.

\subsubsection{Detected combinations of OMPs}

Paracetamol (acetaminophen), phenazone, clofibric acid, primidone, triazines, triazoles, and mecoprop (MCPP) were commonly observed together in the wells of the upper aquifers close to a disused waste disposal site within the urban area. Water levels of the upper aquifers indicate a groundwater divide in the area of the waste disposal. Therefore, contaminants were found north and south of the disposal site. Close to the waste disposal the concentrations of the above mentioned OMPs ranged from 15 up to $>1000 \mathrm{ng} \mathrm{L}^{-1}$. Measured redox potential values were significantly lower close to the waste disposal $(100-200 \mathrm{mV})$ than in greater distance $(>300 \mathrm{mV})$. The detection of these OMPs close to the waste disposal refers to the persistance of the OMPs under the observed reductive conditions. Since the application of primidone is decreasing over the last two decades (Müller et al. 2012) and phenazone is currently not applied in appreciable amounts the input via the disused waste disposal is evident.

Metoprolol in combination with sulfamethoxazole (SMX), carbamazepine, primidone, and isoproturon was repeatedly detected in the well of the z1-aquifer at the south city limit and with lower concentrations at a small spring. The well is located close to various potential input sources such as a former waste disposal, an infiltrating river, a main wastewater conduit, a former wastewater treatment plant and a fault zone. Therefore, identifying a clear source is not possible. The correlation between SMX and carbamazepine is reported for a river in Germany and interpreted as residues of wastewater (Nödler et al. 2011). Carbamazepine is highly persistent in the environment (Clara et al. 2004) and can be used to investigate the influence of sewer leakages to the groundwater (Wolf et al. 2012). The concentrations of carbamazepine, primidone and SMX with its degradation products desamino-SMX and 4-nitro-SMX in the well were higher than in the river water, indicating input by the wastewater system (conduit or former treatment plant) is more likely than by the river or the waste disposal. 


\subsubsection{Environmental implication of detected OMPs}

In Germany a threshold value for drinking water respectively a benchmark for groundwater contamination is defined with $100 \mathrm{ng} \mathrm{L}^{-1}$ for each herbicide. For the analyzed pharmaceuticals and triazoles without defined threshold values practical benchmarks of also $100 \mathrm{ng} \mathrm{L}^{-1}$ are recommended by the Federal Environmental Agency. Exceedings of this value by triazines, mecoprop, primidone and paracetamol were indeed observed but are limited to the proximate surrounding of a waste disposal and individual sampling points. The concentrations in drinking water supply wells and springs were in all cases below $100 \mathrm{ng} \mathrm{L}^{-1}$. Toxicologically derived provisional guideline values for triazoles, carbamazepine, diuron, clofibric acid and phenazone are in the range of micrograms up to milligrams per liter (Schriks et al. 2010) and were not exceeded in the study area.

\subsection{Conclusions}

Most frequently detected OMPs in groundwater samples are triazines and triazoles. In the urban area the input of triazines, triazoles and other OMPs is associated with disused waste disposals (Fig. 3.4). Further triazoles are introduced through an effluent river. Additional input of triazines in the agricultural areas may be indicated by different fractions of atrazine and its degradation products. OMPs distinguish between the North and the South main spring areas with respect to the number of detected OMPs, OMP combinations, occurrence of special compounds like tamoxifen and concentrations of triazoles and triazines. This indicates a water divide south of the urban area. Generally, the upper aquifers exhibit a higher number and higher seasonal variety of OMPs than the lower aquifer and the main spring areas. The sporadic occurrence of some compounds such as caffeine or tamoxifen is only pronounced in the shallow aquifers. 


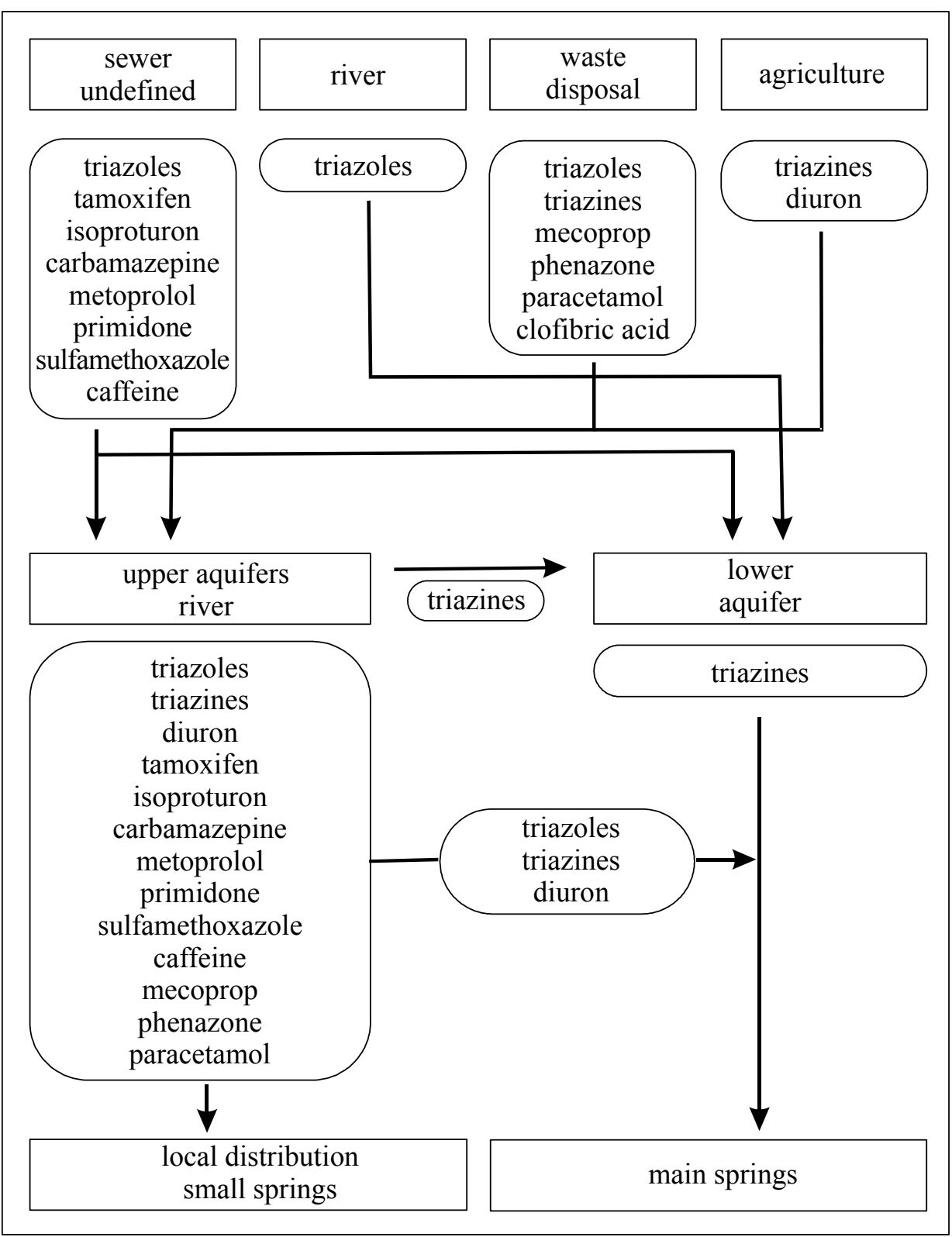

Fig. 3.4: Main sources of OMPs and principles of transport

Regarding prescribed DDDs, biodegradable substances like ibuprofen are underrepresented in groundwater while the occurrence of other pharmaceuticals like carbamazepine, metoprolol or primidone is greater than their prescription would suggest. However, data suggest that metoprolol is better attenuated in groundwater than carbamazepine. Phenazone originates from a disused waste disposal and is showing a persistency for more than three decades under the observed redox conditions. Some cationic compounds such as tamoxifen are only detected during periods of raised groundwater recharge in winter or spring months. 
Triazines occur also in the deep z1-aquifer in areas which are covered by the upper aquifers. Therefore, aquifer connections must exist. Since the application of atrazine as a herbicide has been forbidden since 1992 the detection of triazines is an indicator for residence times in the subsurface of more than 20 years. The occurrence of OMPs in the lower aquifer, solely limited to triazines, indicates limited aquifer connections and demonstrates a residence time of several years for vertical transport into the deep aquifer.

\subsection{Acknowledgements}

This work is funded by the Hessisches Landesamt für Umwelt und Geologie HLUG, Wiesbaden, as well as Energie Waldeck-Frankenberg EWF GmbH, Korbach. Therefore we would like to thank Inga Schlösser-Kluger and Margret Jaeger-Wunderer (HLUG) as well as Stephan Schaller and Friedrich Wilke (EWF) for providing the funds. We express our gratitude to Valentina Coca of WIdO for supplying statistical data of pharmaceutical daily doses and the Federal Ministry of Education and Research promotional reference No. 02WRS1277A, AGRO “Risikomanagement von Spurenstoffen und Krankheitserregern in ländlichen Gebieten".

\subsection{References}

Agverted, J., Rügge, K., Barker, J.F.: Transformation of the herbicides MCPP and atrazine under natural aquifer conditions. Ground Water 30, 500-506 (1992)

Ashton, D., Hilton, M., Thomas, K.V.: Investigating the environmental transport of human pharmaceuticals to streams in the United Kingdom. Sci. Total Environ. 333, 167-184 (2004)

Atkinson, T.C.: Diffuse flow and conduit flow in limestone terrain in the Mendip Hills, Somerset (Great Britain). J. Hydrol. 35, 93-110 (1977)

Buser, H.R., Poiger, T., Müller, M.D.: Occurrence and environmental behavior of the chiral pharmaceutical drug ibuprofen in surface waters and in wastewater. Environ. Sci.

Technol. 33, 2529-2535 (1999) 
Clara, M., Strenn, B., Kreuzinger, N.: Carbamazepine as a possible anthropogenic marker in the aquatic environment: investigations on the behaviour of carbamazepine in wastewater treatment and during groundwater infiltration. Water Res. 38, 947-954 (2004)

Erickson, L.E., Lee, K.H., Sumner, D.D.: Degradation of atrazine and related s-triazines. Crit. Rev. Env. Contr. 19, 1-14 (1989)

Estévez, E., Carmen Cabrera del, M., Molina-Díaz, A., Robles-Molina, J., Pino Palacios-Díaz del, M.: Screening of emerging contaminants and priority substances (2008/105/EC) in reclaimed water for irrigation and groundwater in a volcanic aquifer (Gran Canaria, Canary Islands, Spain). Sci. Total Environ. 433, 538-546 (2012)

Geller, A.: Studies of degradation atrazine by bacterial communities enriched from various biotopes. Arch. Environ. Con. and Tox. 9, 289-305 (1980)

Heinz, B., Birk. S., Liedl, R., Geyer, T., Straub, K.L., Andresen, J., Bester, K., Kappler, A.: Water quality deterioration at a karst spring (Gallusquelle,Germany) due to combined sewer overflow: evidence of bacterial and micro-pollutant contamination. Environ. Geol. 57, 797-808 (2009)

Hilal, S., Karickhoff, S.W., Carreira, L.A.: A rigorous test for SPARC's chemical reactivity models: Estimation of more than 4300 ionization pKa's. Quant. Struc.-Act. Rel. 14, 348-355 (1995)

Hillebrand, O., Nödler, K., Licha, T., Sauter, M., Geyer, T.: Caffeine as an indicator for the quantification of untreated wastewater in karst systems. Water Res. 46, 395-402 (2012)

Hölting. B., Matthess, G.: Ein Sporentriftversuch im Zechstein von Korbach/ Hessen. Notizbl. HlfB 91, 237-255 (1963)

Kolpin, D.W., Furlong, E.T., Meyer, M.T., Thurman, E.M., Zaugg, S.D., Barber, L.B., Buxton, H.T.: Pharmaceuticals, hormones, and other organic wastewater contaminants in U.S. streams, 1999-2000: A national reconnaissance. Environ. Sci. Technol. 36, 1202-1211 (2002)

Kulick, J.: Erläuterungen zur Geologischen Karte von Hessen 1:25.000, Blatt 4719 Korbach. 2. Aufl., 272 p.; Wiesbaden (1997) 
3 Occurrence and spatial distribution of organic micro-pollutants in a complex hydrogeological karst system during low flow and high flow periods, results of a two-year study

McEvoy, G.K. editor: American Hospital Formulary Service-Drug Information 2003 (Plus Supplements). 1770 p.; American Society of Health-System Pharmacists Inc., Bethesda MD (2003)

Menning, M., Hendrich, A.. Stratigraphische Tabelle von Deutschland 2002.; Tafel 96x130 cm oder Falt-Tafel A4. Deutsche Stratigraphische Kommission, Potsdam (Geoforschungszentrum), Frankfurt a. M. (Forsch.-Inst. Senckenberg) (2002)

Metcalfe, C.D., Beddows ,P.A., Bouchot, G.G., Metcalfe, T.L., Li, H., Van Lavieren, H.: Contaminants in the coastal karst aquifer system along the Caribbean coast of the Yucatan Peninsula, Mexico. Environ. Pollut. 159, 991-997 (2011)

Meyer, A.H., Penning, H., Elsner, M.: C and N fractionation suggests similar mechanisms of microbial atrazine transformation despite involvement of different enzymes (AtzA and TrzN). Environ. Sci. Technol. 43, 8079-8085 (2009)

Müller, B., Scheytt, T., Asbrand, M., Mross de Casas, A.: Pharmaceuticals as indictors of sewage-influenced groundwater. Hydrogeol. J. 20, 1117-1129 (2012)

Nödler, K., Licha, T., Bester, K., Sauter, M.: Development of a multi-residue analytical method, based on liquid chromatography-tandem mass spectrometry, for the simultaneous determination of 46 micro-contaminants in aqueous samples. J. Chromatogr. A 1217, 6511-6521 (2010)

Nödler, K., Licha, T., Fischer, S., Barbieri, M., Pérez, S.: Evidence for microbially mediated abiotic formation of reversible and non-reversible sulfamethoxazole transformation products during denitrification. Water Res. 46, 2131-2139 (2012)

Nödler, K., Licha, T., Fischer, S., Wagner, B., Sauter, M.: A case study on the correlation of micro-contaminants and potassium in the Leine River (Germany). Appl. Geochem. 26, 2172-2180 (2011)

Richardson, M.L., Bowron, J.M.: The fate of pharmaceutical chemicals in the aquatic environment. J. Pharm. Pharmacol. 37, 1-12 (1985) 
Schriks, M., Heringa, M.B., van der Kooi. M.M.E., de Voogt, P., van Wezel, A.P.: Toxicological relevance of emerging contaminants for drinking water quality. Water Res. 44, 461-476 (2010)

Schwarzenbach, R.P., Escher, B.I., Fenner, K., Hofstetter, T.B., Johnson, C.A., von Gunten, U., Wehrli, B.: The challenge of micropollutants in aquatic systems. Science 313, 1072-1077 (2006)

Ternes, T.A.: Occurrence of drugs in German sewage treatment plants and rivers. Water Res. , 32, 3245-3260 (1998)

White, W.B.: Conceptual models for carbonate aquifers. Ground Water 7, 15-21 (1969)

Wolf, L., Zwiener, C., Zemann, M.: Tracking artificial sweeteners and pharmaceuticals introduced into urban groundwater by leaking sewer networks. Sci. Total Environ. 430, 8-19 (2012) 



\section{Evaluation and application of organic micro-pollutants (OMPs) as indicators in karst system characterization}

Roland Reh ${ }^{\mathrm{a}}$, Tobias Licha ${ }^{\mathrm{a}}$, Karsten Nödler ${ }^{\mathrm{a}}$, Tobias Geyer ${ }^{\mathrm{a}, \mathrm{b}}$, Martin Sauter

Citation:

Reh, R., Licha, T., Nödler, K., Geyer, T., Sauter, M.: Evaluation and application of organic micro-pollutants (OMPs) as indicators in karst system characterization.

Environ. Sci. Pollut. Res. DOI 10.1007/s11356-014-3676-z (2014)

http://dx.doi.org/10.1007/s11356-014-3676-z

(C) Springer-Verlag Berlin Heidelberg 2014

With kind permission of Springer Science+Business Media

aUniversität Göttingen, Geowissenschaftliches Zentrum, Angewandte Geologie, Goldschmidtstraße 3, 37077 Göttingen, Deutschland

${ }^{\mathrm{b}}$ Landesamt für Geologie, Rohstoffe und Bergbau (LGRB) im Regierungspräsidium Freiburg, Albertstraße 5, 79104 Freiburg im Breisgau, Deutschland 


\section{Abstract}

This study presents chances and challenges associated with the application of organic micropollutants (OMPs) as indicators in karst system characterization. The methodology and options of possible indications were evaluated based on the interpretation of the spatial distribution of 54 compounds in groundwater in combination with a complex geological setting consisting of multiple aquifer horizons and tectonic faults. A high variety of OMPs are released mainly in an urban area leading to concentrations of several $n g \mathrm{~L}^{-1}$ up to $\mu \mathrm{g} \mathrm{L}^{-1}$, which are detectable by an HPLC-MS/MS-method. Since characteristic patterns of spatial distribution were repeatedly observed during a two years observation period, important criteria of the aforementioned indicator application are fulfilled. Triazoles, compounds with recent high emission rates, could be successfully applied for the identification of flow directions and the delineation of catchment areas. Concentrations and the number of OMPs are believed to depend on properties of covering rock layers. Therefore, OMPs can also be used as a validation tool for vulnerability mapping. Compounds, such as triazines, persistent in the system for more than two decades, demonstrate the interaction between different parts of the aquifer system and the hydraulic characteristics of a tectonic fault zone. Such indicator potentials complement those of artificial tracer tests. Point sources of OMPs and their impact on groundwater could be identified qualitatively. In combination with the interpretation of the geological setting the distribution of OMPs provides essential information for the development of a conceptual hydrogeological model.

\subsection{Introduction}

Karst aquifers are generally characterized as prolific water resources systems with substantial hydraulic conductivity and large contrasts between the conductivity of the draining conduit features and the matrix storage system. Often, the catchments are large and drain to individual springs with highly variable discharge. Due to these properties, approximately $25 \%$ of the worldwide fresh water demand is abstracted from karst aquifers (Ford \& Williams 2007). On the other hand, highly permeable fractures, conduits and dolines rapidly convey contaminants across the vadose zone with a resulting high vulnerability (COST 2003). Therefore, knowledge on the location and the extent of subsurface catchments is not only a pre-requisite for the delineation of groundwater protection zones but also a vital tool for the sustainable management of karst aquifers as important drinking water resources. Further- 
more, the recent water safety plan from the World Health Organization emphasizes the need for process and system understanding on catchment scale (WHO 2011). However, the characterization of fractured and karstified aquifer systems is a challenging task. This is mainly due to their large-scale hydraulic heterogeneities, the problems in detecting and characterizing the dominating karst conduits and the unknown location and extent of the subsurface karst groundwater catchments (Geyer et al. 2013). Further, the large contrast between long residence times in fissured matrix blocks and the observed rapid flow velocities in highly permeable fractures or karst conduits need to be both reconciled in a conceptual model. The results from classical hydrogeological methods (e.g. hydraulic tests) in the characterization of these types of aquifer systems are often not representative and usually limited to only a small part of the whole aquifer system (Worthington 2003, Sauter 1992). Artificial tracer tests are a traditional method for aquifer characterization. These tests are suitable for e.g., the identification of point-point-connections and the determination of flow and transport parameters (Field and Pinsky 2000, Hillebrand et al. 2012a). However, artificial tracer tests require a suitable injection location, and, if applied in a large area or with the focus on long term studies and low flow velocities, substantial observation efforts (Käss and Behrens 1998, Goldscheider et al. 2008, Licha et al. 2013). Further, such tests may be in conflict with public water supply operations. The above reasons highlight the necessity for the development of additional characterization techniques, which allow the aquifer characterization on catchment scale and processes on different time scales with reasonable sampling and analytical effort.

Many studies within the last two decades have shown the frequent occurrence of numerous organic micro-pollutants (OMPs) in the aquatic environment (e.g. Schwarzenbach et al. 2006, Sacher et al. 2008) including recent studies on karst aquifer systems (Estévez et al. 2012, Hillebrand et al. 2012b, Morasch 2013, Reh et al. 2013). This widespread occurrence opens the possibility of using OMPs as specific indicators for flow, transport and source delineation in aquatic systems. They were already successfully applied in surface waters as indicators for the identification of input functions, transport behavior or compound persistency (Daneshvar et al. 2010, Kolpin et al. 2002), and in riverbank filtration systems (Zuehlke et al. 2007).

The application of OMPs as indicators for the characterization of fractured and karstified aquifer systems is still relatively new and, if applied, limited to a low number of observation points (Metcalfe et al. 2011). Current applications deal with the characterization of single subsurface flow pathways between an infiltration point and an outlet, e.g., a karst spring (Heinz et al. 2009, Hillebrand et al. 2012b). In order to extend the application spectrum of OMPs in fractured and karstified aquifer systems the aims of this study are, firstly, the 
general evaluation of OMPs as possible indicators for transport processes on large scale (greater than catchment scale) by studying the distribution pattern of OMPs on 51 sampling points distributed over the whole study area and, secondly, the examination of the ability to derive aquifer or even karst system properties from the spatial distribution or seasonal variation of OMPs in combination with the hydrogeological setting and other features of the study area. Furthermore, the indicator potential of OMPs in such systems is compared to that of classical indicators such as hydrochemical facies and contaminants such as chlorinated hydrocarbons (CHCs).

\subsection{Materials and methods}

\subsubsection{Study area}

The study area $\left(65 \mathrm{~km}^{2}\right)$, located in the central part of Germany (North Hesse), is characterized by a tectonically faulted aquifer system consisting of three moderately karstified carbonate aquifer horizons and a perched sandstone aquifer. Land use is dominated by agriculture and forestry. A town with 24,000 inhabitants and industrial areas, including a site, known to be contaminated with $\mathrm{CHCs}$, is located in the catchment area. Several decommissioned waste disposal sites are also located in the urban area (Fig. 4.1).

The three carbonate aquifer horizons are developed mainly within the Zechstein sequences z1-z3 (Menning \& Hendrich 2002), associated with the eastern rim of the Rhenish massif (Kulick 1997). Intercalated formations of shale rocks and sandstones act as aquitards. A low conductive sandstone aquifer is developed at the top of the Zechstein sequences $\mathrm{z} 3-\mathrm{zFb}$ (Fig. 4.1). The Zechstein sequences are followed by mainly fine-grained sandstones of the Lower Buntsandstein. For the purpose of this study the z1-carbonate aquifer is defined as "Lower Aquifer" while the carbonate aquifer of the z3-sequences is termed "Upper Aquifer" and the low conductive sandstone aquifer of the upper Zechstein sequences is the "Perched Aquifer". The carbonates of the z1- and the z3-sequences with karst features, such as solution enlarged fractures and large springs, are important for groundwater flow. Due to an absence of suitable observation boreholes no reliable information about the z2-carbonate are available. 


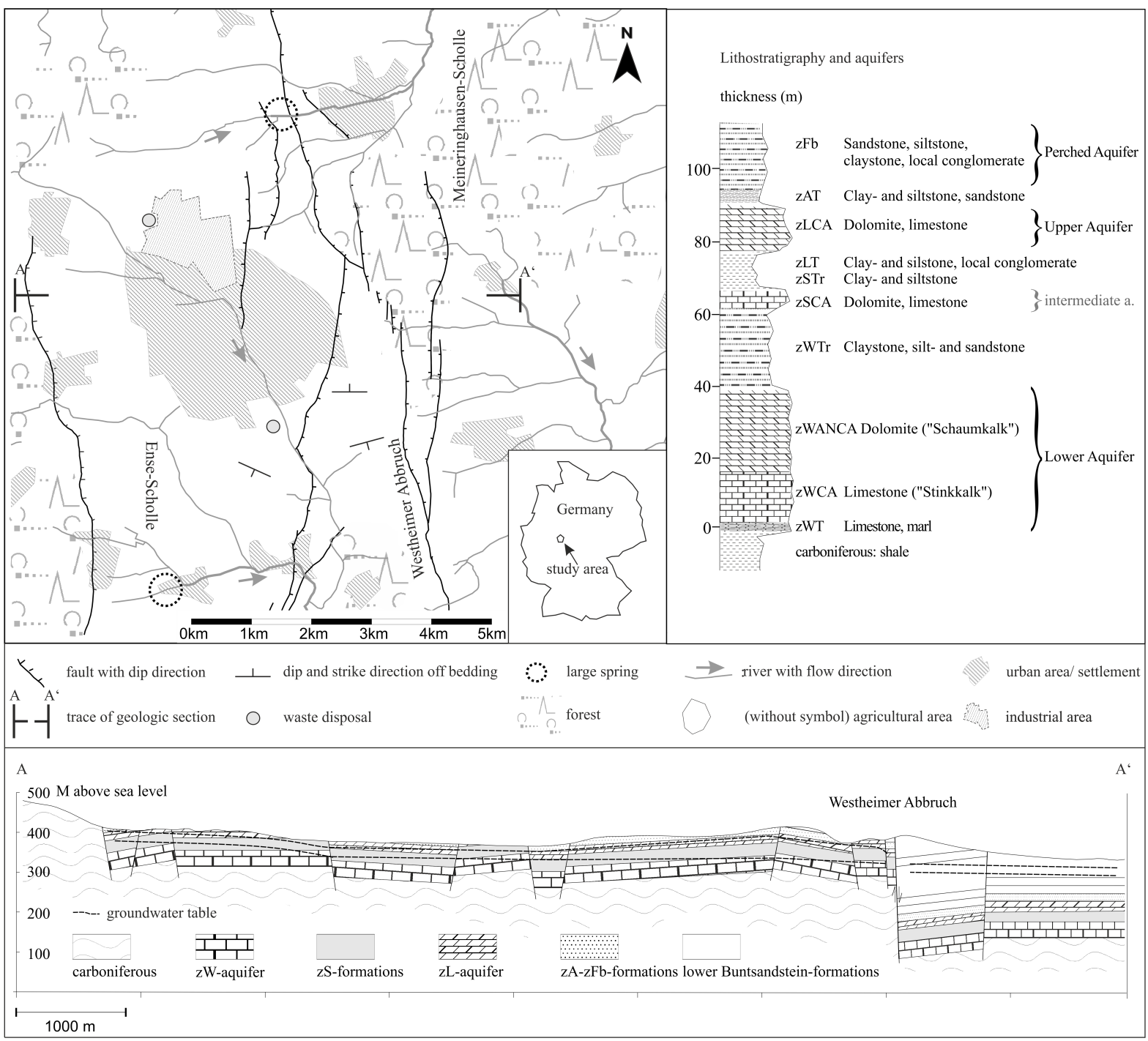

Fig. 4.1: Characteristics of the study area (Reh et al. 2013), geological setting and stratigraphy of the Zechstein sequences (z1-zFb)

As a result of a North-South striking tectonic fault system (Westheimer Abbruch) the investigation area is divided into two main tectonic blocks, Ense-Scholle in the West and Meineringhausen-Scholle in the East (Schraft et al. 2002). The fault displacement is ca. $100 \mathrm{~m}$ to $150 \mathrm{~m}$. The Ense-Scholle is characterized by Zechstein sediments, exposed at its surface, younger sediments of the Lower Buntsandstein cover the Meineringhausen-Scholle. Both tectonic blocks are sub-divided by NW and NE striking subfaults with small displacements of 10 $20 \mathrm{~m}$ (Fig. 4.1) resulting in a subsystem of horst and graben structures.

In the northern part of the Ense-Scholle the main dip of the bedding is North with a small angle of $3-10^{\circ}$. South of the city an anticline with weak dipping flanks can be observed in a decommissioned quarry and along a riverbed (Fig. 4.1). In the southern part of the Ense- 
Scholle the dip is mainly Southwest. This change in direction of the dip can also be identified from geological map and borehole log information. The tectonic block "MeineringhausenScholle" declines towards the North (Kulick 1997).

The urban area is located on a plateau, which is drained by rivers in the North, East and South. Large springs with an average discharge of ca. $75 \mathrm{~L} \mathrm{~s}^{-1}$ each are located in the North and the South of the study area. Discharge of resulting rivers increases along a flow path of $9 \mathrm{~km}$ to an average of $310 \mathrm{~L} \mathrm{~s}^{-1}$ (North) and over a distance of $2 \mathrm{~km}$ to an average of $250 \mathrm{~L} \mathrm{~s}^{-1}$ (South) indicating groundwater discharge into the river. East of the fault Westheimer Abbruch, several small springs with discharges of maximum $5 \mathrm{~L} \mathrm{~s}^{-1}$ feed a river with a total discharge of $100 \mathrm{~L} \mathrm{~s}^{-1}$ at the southern border of the study area. Between spring and late summer groundwater levels vary within a fluctuation range of 0.2 and $2.0 \mathrm{~m}$. The discharge of the large springs decreases by ca. 30 to $60 \%$ during this period. This fluctuation leads to the drying up of several small springs discharging from the Upper Aquifer and the Perched Aquifer during late summer and autumn. The water levels of the aquifers exhibit different heights with a $30 \mathrm{~m}$ higher hydrostatic level in the Upper Aquifer compared to the Lower Aquifer in the central study area. From the Ense-Scholle to the Meineringhausen-Scholle the water level exhibits a gradient of approximately 0.01. Springs in the Rhenish Massif feed two rivers with a low discharge $\left(<10 \mathrm{~L} \mathrm{~s}^{-1}\right)$ crossing the study area from Northwest to Southeast and loose water into the aquifer system. The eastern river represents the main drainage from the urban area.

\subsubsection{Sampling}

This evaluation is based on samples of different types of observation points, which include wells, springs, and rivers. Samples were taken for the analysis of OMPs (Reh et al. 2013) as well as for the analysis of additional parameters (major ions, CHCs). Four sampling campaigns were conducted during low flow and high flow periods of the years 2010 and 2011 at 27 to a maximum of 51 sampling points (Reh et al. 2013). Most of the sampling points (23) are associated with the Upper Aquifer and the Perched Aquifer, while 9 sampling points are located in the Lower aquifer. A number of 11 sampling points receive water from both carbonate aquifers or they cannot be attributed to any specific aquifer. Two of them are located immediately at the western boundary of the carbonate aquifers. Eight further observation points are located along the rivers. The thin z2-carbonate is not represented by any specific sampling point. On-site parameters (temperature, $\mathrm{pH}$, electric conductivity, redoxpotential, concentrations of dissolved oxygen) were measured during sampling. Grab 
samples, taken from spring discharge and surface waters, were filled directly into sample bottles. Public supply and private abstraction wells were sampled directly from the rising main during their continuous production. In observation wells a submerged pump was installed for well purging purposes and the sample was taken immediately afterwards using a PTFE bailer. Samples for the analysis of OMPs and CHCs were filled into $500 \mathrm{~mL}$ and $1000 \mathrm{~mL}$ glass bottles with screw caps. PE-bottles $(10 \mathrm{~mL})$ were used for samples with the aim of determining major ions. The samples were transported to the laboratory in a dark, cooled box. Solid phase extraction (SPE) for the analysis of OMPs and the analysis of CHCs were conducted within 24 hours after sampling. The SPE cartridges were stored at $-18{ }^{\circ} \mathrm{C}$ until analysis. This method was demonstrated to be an adequate approach regarding analyte stability (Hillebrand et al. 2013).

\subsubsection{Chemical analysis}

A multi-residue analytical method based on high-performance liquid chromatographic chromatography separation with subsequent tandem mass spectrometric detection (HPLCMS/MS) was employed for the analysis of OMPs. Details regarding the extraction and analysis were published previously (Nödler et al. 2010, Reh et al. 2013). The method quantitation limits (MQL) reported therein are between 1.2 and $28 \mathrm{ng} \mathrm{L}^{-1}$. The analysis comprises the simultaneous quantitation of 54 OMPs.

Main water chemistry was determined by ion chromatography (IC). Prior to IC, samples were filtered (Whatman Anotop $10 \mathrm{IC}, 0.2 \mu \mathrm{m})$. The determination of inorganic cations $\left(\mathrm{Na}^{+}\right.$, $\left.\mathrm{K}^{+}, \mathrm{Mg}^{2+}, \mathrm{Ca}^{2+}\right)$ was conducted on a DX-500 Ion Chromatography System with conductometric detection and electrochemical suppression (Dionex, Sunnyvale, CA). This isocratic system runs with $20 \mathrm{mM}$ methanesulfonic acid as the eluent with a flow rate of $0.45 \mathrm{~mL} \mathrm{~min}{ }^{-1}$ at a temperature of $44^{\circ} \mathrm{C}$ using an IonPac CS12A ( $3 \times 150 \mathrm{~mm}$, Dionex) column. For the determination of anions $\left(\mathrm{Cl}^{-}, \mathrm{NO}_{3}{ }^{-}, \mathrm{SO}_{4}{ }^{2-}\right)$ a Dionex DX-320 with conductometric detection and electrochemical suppression (Dionex, Sunnyvale, CA), a Dionex IonPac AS11-HC (2 x $250 \mathrm{~mm}$ ) column and a $22 \mathrm{mM} \mathrm{KOH}$ as eluent (isocratic separation at $30^{\circ} \mathrm{C}$ ) were used. For these separations, a flow rate of $0.38 \mathrm{~mL} \mathrm{~min}^{-1}$ was applied.

CHCs were analyzed by gas chromatography with mass spectrometric detection (GC/MS) according to DIN EN ISO 10301 respectively DIN 38413-P2. The method quantitation limit for 15 detectable CHCs was $100 \mathrm{ng} \mathrm{L}^{-1}$. 


\subsection{Results and discussion}

\subsubsection{Evaluation of OMPs as indicators}

Geogenic background concentrations for OMPs can be excluded as they are solely released by human activity in our climate zone. This fact considerably increases their sensitivity with respect to their capability of detecting processes in aquifers and a direct interpretation of even low concentrations ( $\mathrm{ng} \mathrm{L}^{-1}$ ) becomes possible. In contrast to chlorinated solvents, sampling losses in outflowing springs can be neglected because the analyzed OMPs are not regarded as volatile due to high boiling points respectively low henrys law constants (EU 2004). Therefore, OMPs are suitable indicators and were applied as such for source apportionment in earlier studies (e.g., Wolf et al. 2004, Müller et al. 2012, Metcalfe et al. 2011). Massmann et al. (2008) demonstrated also the indication of processes by certain OMPs, which are dependent on the prevailing redox conditions. Consequently, for these applications the OMPs must be specific for a certain source or process. However, for the characterization of a complex karst aquifer system some requirements on the OMPs have to be fulfilled, because indicators must reflect hydraulic connections on a small scale as well as large scale including transient states. For such challenges, OMPs are only really useful and powerful indicators if they show temporal and/or spatial variations (Stuart et al. 2014). Additionally, the selected analytes should include parameters, which allow for more than source specific interpretations. Therefore, the analyzed OMPs in this study include pharmaceuticals, pesticides, corrosion inhibitors, and everyday products such as caffeine. Additionally, metabolites of some substances are included. The compounds exhibit different characteristics regarding degradability and consumed amounts as well as periods of application. For instance, triazines or certain pharmaceuticals are known to be persistent, highly mobile in aquatic environments (Tappe et al. 2002), and may, therefore, indicate long-term behavior in contrast to biodegradable compounds, such as caffeine, which are only detectable in groundwater episodically (Hillebrand et al. 2012a). Some compounds contain temporal information, as they are not constantly used. This applies to pharmaceuticals or herbicides, which were replaced either by more effective compounds or due to regulatory affairs. Our preliminary evaluation of data demonstrated a frequent occurrence of OMPs in the study area (Reh et al. 2013). Already the number of OMPs per sampling point shows a specific distribution pattern, with seasonal variations recurring in four sampling campaigns. For illustration (Fig. 4.2) the OMPs are classified into 4 fields of application (pesticides including biocides, pharmaceuticals, corrosion inhibitors and caffeine). Detected metabolites are allocated to the application of their basic compound. In the urban area generally the largest number and highest varia- 
tion of compounds were observed. At most of the urban sampling locations, OMPs of the categories pesticides/ biocides, pharmaceuticals and corrosion inhibitors were the dominating compounds, while the agricultural areas in the South and in the East show a low number of OMPs, predominantly pesticides.
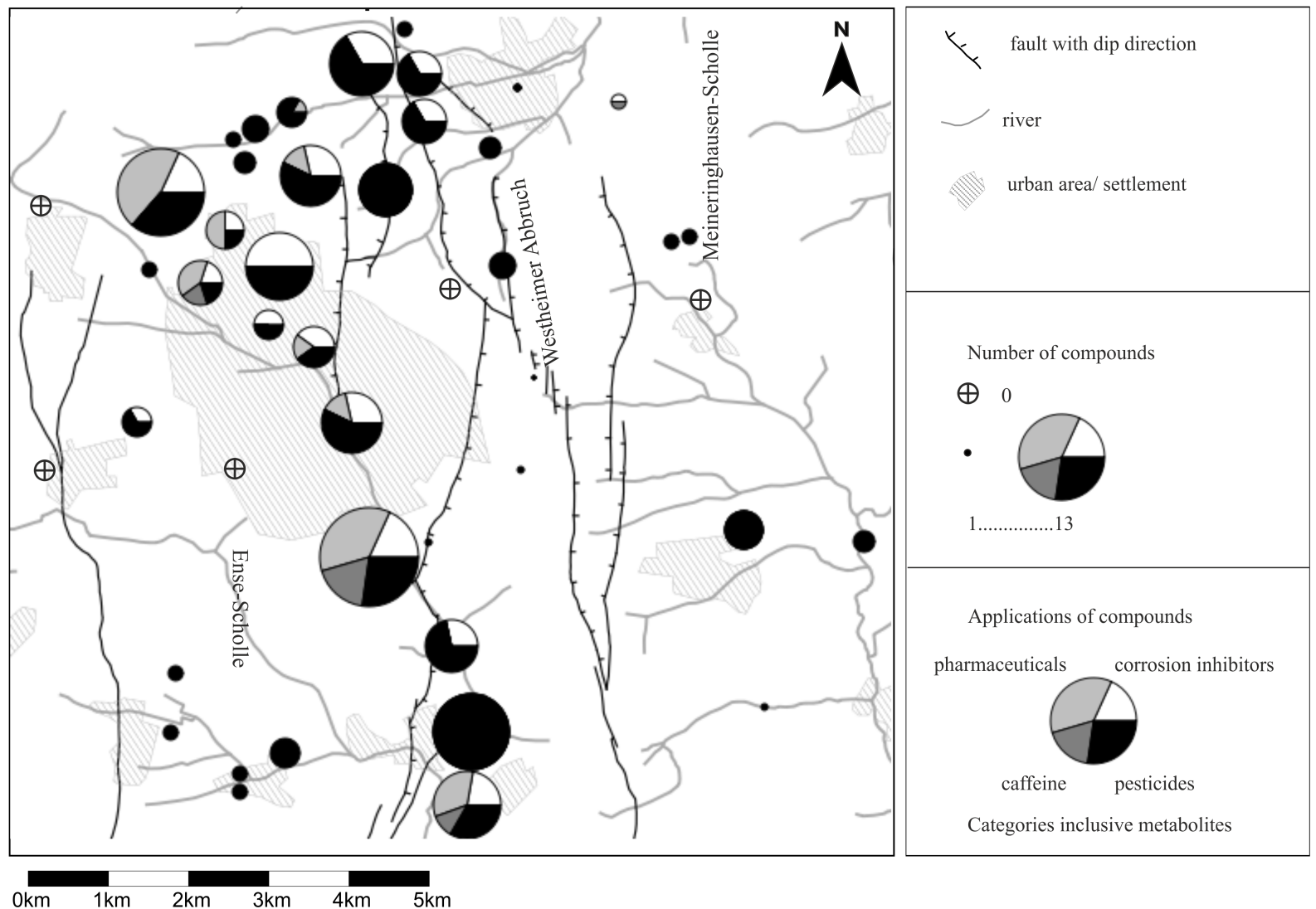

Fig. 4.2: Spatial distribution of the number of detected OMPs per sampling point, assigned to different types of applications, size of the pie charts depends on the detected number of OMPs

An extended inspection reveals a distinct spatial and seasonal distribution of compounds respectively compound classes:

- As reported in Reh et al. 2013, triazines showed a broad spatial distribution, although the application of atrazine as a pesticide is not permitted in Germany since 1992. The ratio of atrazine to triazine metabolites desethylatrazine (DEA) and desisopropylatrazine (DIA) was different with respect to their associated source. One identified point source of DIA is an old waste disposal while triazines released in agricultural areas seem to be DEA dominated. 
- Triazoles are still in use for a wide range of applications. From this substance group 1H-benzotriazole (BT) as well as tolyltriazole were analyzed and detected repeatedly in a large number of sampling points. BT has a high solubility in water $\left(28 \mathrm{~g} \mathrm{~L}^{-1}\right)$ and a relatively low octanol-water distribution coefficient (log $\mathrm{K}_{\mathrm{OW}}$ ) of 1.23 (Hart et al. 2004). It is considered as little biodegradable (Giger et al. 2006). With such characteristics, a high mobility in both surface water and groundwater can be expected and the compound class fulfills the indicator requirements. Accordingly, BT is detected in German river systems (Nödler et al. 2011) and can be traced until the North Sea (Wolschke et al. 2011).

- The different applications and consequently different input functions or locations of triazines and triazoles are reflected by chemographs. As shown in Fig. 4.3 the deviation of tolyltriazole concentrations seems to be independent of seasonal influences. In contrast, the deviation of DEA concentrations correlates with changing spring discharges. During high flow periods the concentrations of DEA were approximately twice as high compared to low flow periods. The variation is more distinctive in the upper aquifers than in the Lower Aquifer. While tolyltriazole may be discharged locally by influent rivers or the sewer system, DEA could has been released extensively from agricultural areas resulting from the degradation of atrazine (ATR) applied $\geq 20$ years ago (Hillebrand et al. 2014).

- The combination of the pharmaceuticals paracetamol, phenazone, primidone and the biocide mecoprop is attributed to a decommissioned waste disposal site. 


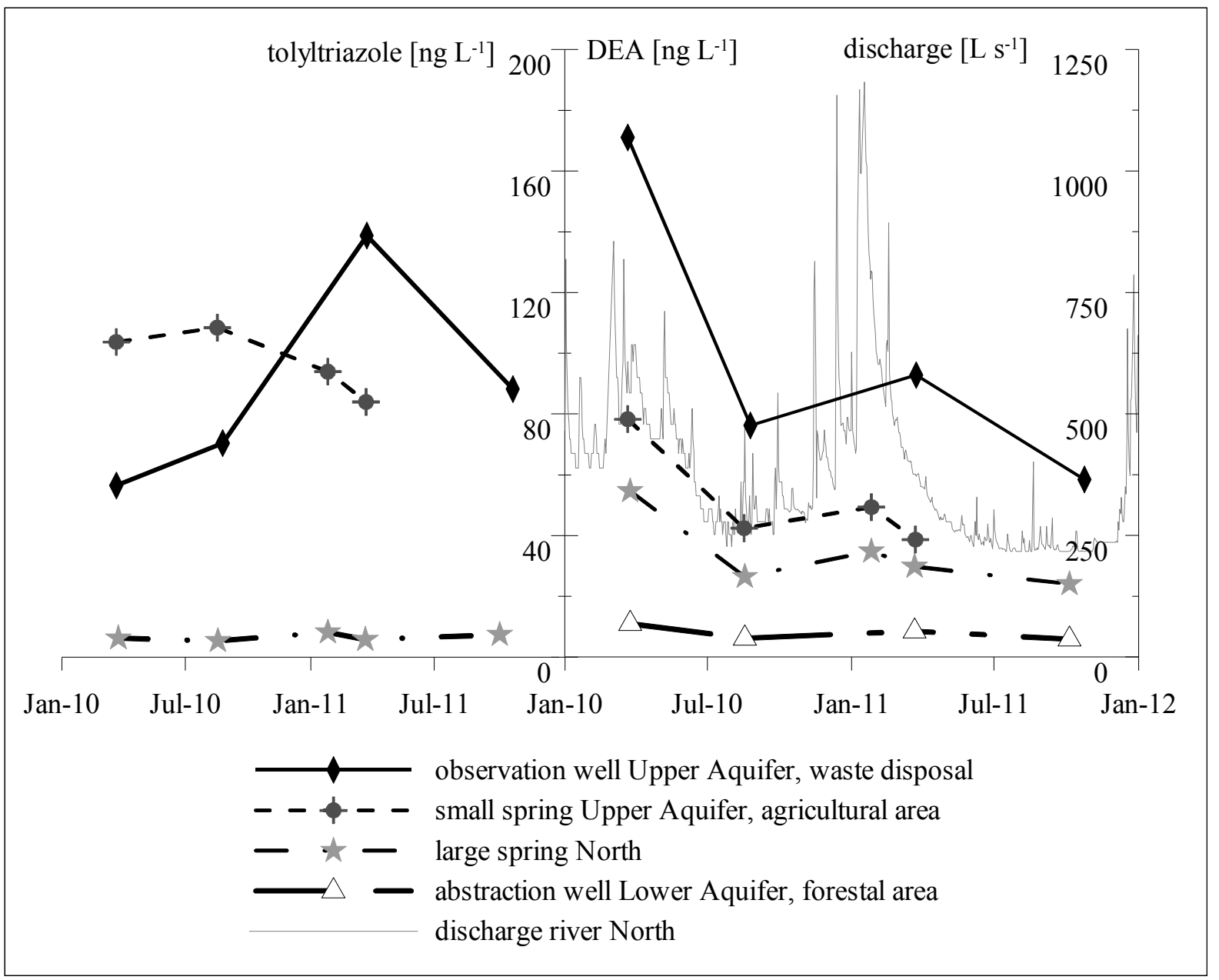

Fig. 4.3: Seasonal deviation of tolyltriazole and DEA concentrations in different types of sampling points, areas and aquifers, in relation to the discharge of the river in the North

The vertical sequence of the water bodies shows different combinations of the most frequently detected compounds (s. Tab.4.1). While in the rivers and shallow aquifers readily biodegradable compounds, such as caffeine, were frequently detected, the Lower Aquifer and the large springs are only dominated by compounds considered as substances with a high persistence in the environment, e.g., triazines (Tappe et al. 2002), triazoles (Giger et al. 2006), and carbamazepine (Clara et al. 2004). This observation suggests that the spatial as well the temporal distribution of all detected OMPs do not only depend on the distribution of sources but is also a result of the hydrogeological characteristics of the investigated system, e.g., residence times. Consequently, the applicability of OMPs to contribute to a conceptual understanding of the aquifer system is discussed in the following with focus on their qualities compared to classical indicators such as hydrochemical facies and CHCs. 
Tab. 4.1: Most frequently detected OMPs, allocated to the water body of detection

\begin{tabular}{|c|c|c|c|c|c|}
\hline OMP & $\begin{array}{l}\text { Number of } \\
\text { samples }\end{array}$ & $\begin{array}{c}\text { Detection frequency } \\
{[\%]}\end{array}$ & Min. & $\begin{array}{l}\text { Median } \\
\text { concentration }\left[\mathrm{ng} \mathrm{L}^{-1}\right]\end{array}$ & Max. \\
\hline \multicolumn{6}{|l|}{ Rivers: } \\
\hline Desethylatrazine & 19 & 84 & 1.8 & 16 & 190 \\
\hline Atrazine & 19 & 74 & 1.5 & 6.6 & 25 \\
\hline 1H-Benzotriazole & 19 & 68 & 4.9 & 41 & 6500 \\
\hline Tolyltriazole & 19 & 68 & 5.4 & 27 & 300 \\
\hline Caffeine & 19 & 58 & 1.7 & 12 & 160 \\
\hline Ibuprofen & 19 & 32 & 7.4 & 20 & 43 \\
\hline Paraxanthine & 19 & 32 & 6.5 & 13 & 31 \\
\hline Metoprolol & 19 & 32 & 4.6 & 31 & 640 \\
\hline Metazachlor & 19 & 32 & 2.3 & 6.7 & 8.8 \\
\hline Terbuthylazine & 19 & 32 & 3.5 & 11 & 39 \\
\hline \multicolumn{6}{|c|}{ Upper and perched aquifers: } \\
\hline Desethylatrazine & 94 & 68 & 2.2 & 15 & 170 \\
\hline Atrazine & 94 & 53 & 1.3 & 7.3 & 86 \\
\hline 1H-Benzotriazole & 94 & 48 & 3.4 & 45 & 3200 \\
\hline Tolyltriazole & 94 & 33 & 5.3 & 18 & 210 \\
\hline Mecoprop & 94 & 29 & 1.2 & 35 & 2900 \\
\hline Desisopropylatrazine & 94 & 26 & 3.5 & 23 & 590 \\
\hline Phenazone & 94 & 18 & 2.5 & 160 & 6200 \\
\hline Terbuthylazine & 80 & 15 & 3.2 & 6.2 & 93 \\
\hline Caffeine & 94 & 13 & 5 & 9.8 & 84 \\
\hline Carbamazepine & 94 & 13 & 2.4 & 16 & 53 \\
\hline \multicolumn{6}{|c|}{ Lower aquifer and large springs: } \\
\hline Desethylatrazine & 58 & 86 & 1.4 & 17 & 77 \\
\hline Atrazine & 58 & 84 & 1.9 & 6.8 & 35 \\
\hline 1H-Benzotriazole & 58 & 34 & 4.9 & 18 & 460 \\
\hline Tolyltriazole & 58 & 34 & 5.4 & 21 & 190 \\
\hline Diuron & 58 & 24 & 3.3 & 5.1 & 13 \\
\hline Desisopropylatrazine & 58 & 22 & 1.4 & 9.6 & 38 \\
\hline Terbuthylazine & 48 & 17 & 3.8 & 4.4 & 6.0 \\
\hline Carbamazepine & 58 & 16 & 23 & 48 & 350 \\
\hline Primidone & 58 & 16 & 4.6 & 6.9 & 95 \\
\hline Metoprolol & 58 & 14 & 4.9 & 7 & 74 \\
\hline
\end{tabular}




\subsubsection{Application of OMP as indicators in a complex karst system in support of the development of a conceptual flow model}

A major challenge in the characterization of complex fractured aquifers is to improve or develop methods that allow for catchment delineation and vulnerability assessments with comparatively limited effort.

For catchment delineation a sharp contrast in compound occurrences or a significant variation of concentrations between catchments are needed. Such a contrast is detectable among the triazoles. As an example, Fig. 4.4 presents the spatial distribution of $1 \mathrm{H}$-benzotriazole (BT) observed in August 2010. The results of the other sampling campaigns show a similar image. BT exhibits with $41 \%$ a high detection frequency in all analyzed groundwater samples (Reh et al. 2013). BT is a corrosion inhibitor added to cooling water in industrial processes but is also used both as a silver protection agent and as an additive for dishwashing detergents (Giger et al. 2006). In agreement with its application range, BT occurred mainly in the urban area. Its concentrations reach a maximum of $3200 \mathrm{ng} \mathrm{L}^{-1}$ in groundwater. One of the sources is a small river, crossing the urban area from the North to South, which infiltrates into the subsurface with a maximum concentration of $6500 \mathrm{ng} \mathrm{L}^{-1}$. Herewith, the concentrations of BT were much higher in the river than in the analyzed respective groundwater samples. Another area of elevated BT concentrations in groundwater is located north of the city (Fig. 4.4). In contrast, in the southwestern part of the Ense-Scholle and inside the Meineringhausen-Scholle, BT was only detected in a low number of sampling points. This spatial distribution refers to two subsurface water divides south and east of the urban area between the large spring in the North and in the South as well as between the tectonic blocks EnseScholle and Meineringhausen-Scholle. The existence of a boundary within the Ense-Scholle was previously described with an uncertainty of about $1 \mathrm{~km}$ (Hölting \& Matthess 1963). Now, it can be drawn accurately indicated by different concentrations of BT and also by the variety of OMPs in two sampling points, each representing a catchment. The resulting Northwest-Southeast oriented subsurface water divide coincides with a flat syncline of the geological bedding in this area. Exceptions of the described spatial distribution of BT are the rivers, which may be directly influenced by wastewater, and one well belonging to the z3-aquifer within the Ense-Scholle. However, the latter is located in a forest. In this well concentrations between 1600 and $3200 \mathrm{ng} \mathrm{L}^{-1}$ of BT were measured. The reason for this relatively high concentration outside possible sources still remains unclear. 

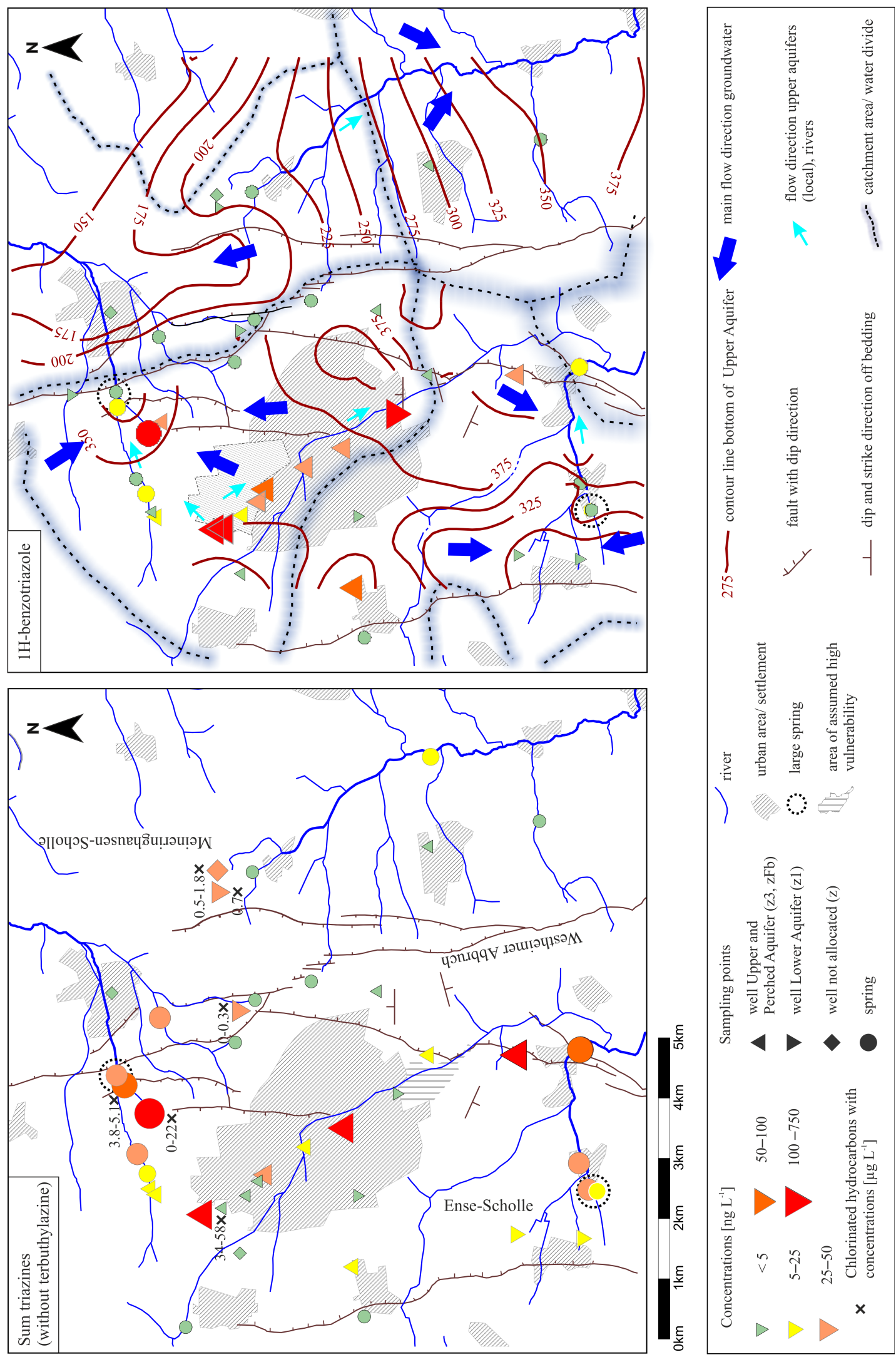

Fig. 4.4: Spatial distribution of triazine concentrations (atrazine, DEA, DIA) and BT concentrations in August 2010, catchment delineation of the spring areas and correlation with base contour lines of the Upper Aquifer 
As demonstrated in Fig. 4.4, triazines (ATR, DEA, DIA) were also widely detected. Unlike triazoles they occurred throughout the whole aquifer system. Due to a missing contrast in occurrence and concentrations the sum of triazines cannot be used for the delineation of the catchments. In numerous studies the ratio of atrazine and metabolites was applied as indicator for subsurface processes (e.g. Adams \& Thurman 1991, Thurman \& Fallon 1996). A spatial variation of the ratios between atrazine and triazine metabolites was earlier identified in the study area (Reh et al. 2013). Highest triazine concentrations are related to an old waste disposal site in the urban area decommissioned more than 30 years ago. Here and in the surrounding industrial area, the ratio of DIA amounts to more than $50 \%$ of the sum of total triazine concentrations (atrazine, DEA, DIA). In contrast, in agricultural areas the triazine composition is clearly DEA dominated (60-90\%). Since DEA and DIA are possible metabolites of numerous triazines (e.g. atrazine, terbuthylazine, simazine, propazine) the degradation processes cannot be identified. However, the contrast in triazine ratios refers to different types of emissions. The large spring in the North exhibits also significant amounts of atrazine as well as DEA and DIA that may indicate a mixing of groundwater of the industrial and the agricultural area. This means, consistent with the performed catchment delineation, that the urban area is located in the catchment of the spring in the North.

Tectonic faults can act as effective preferential flow paths as well as hydraulic barriers. For the development of a conceptional model of the study area the hydraulic characterization of the fault Westheimer Abbruch, separating the carbonate aquifers, is essential. ATR, DEA and DIA are the only OMPs that were repeatedly found in a well developed in the Lower Aquifer immediately west of the Westheimer Abbruch (Fig. 4.4). Here, ATR occurred in the same ratio to DEA and DIA that is characteristic for the triazine composition in the urban area. Consequently, this composition can be seen as an indicator for a connection between the urban area and the well west of the fault. East of the Westheimer Abbruch two wells, situated in a forest, are influenced by ATR and DEA in all sampling campaigns and additionally by DIA in one sampling campaign. Since one of them is only developed in the Lower Aquifer it may be evident that ATR and DEA must have overcome intermediate hydraulic layers. The occurrence of DIA refers to a source of the urban area and would herewith also indicate a connection across the fault zone. On the one hand, due to a variety of possible precursors and the singular detection of DIA this hypothesis may be questioned. On the other hand, in combination with additional information on the hydraulic gradient and the occurrence of $\mathrm{CHCs}$ in the Meineringhausen-Scholle this interpretation is supported. The sparse occurrence of OMPs and the absence of recently used OMPs, representing the urban area, refer to a travel time of more than 20 years and consequently limited aquifer connections. 
As a conventional method for distinguishing different types of groundwater from different aquifers, interpretations of the fractions of major ions may be applied (Furtak \& Langguth 1967). However, these show only low variations in the study area (Fig. 4.5). The mineralogical composition does not differ much between the different aquifer units and catchment areas. Therefore, samples cannot be allocated to individual aquifers or catchments.

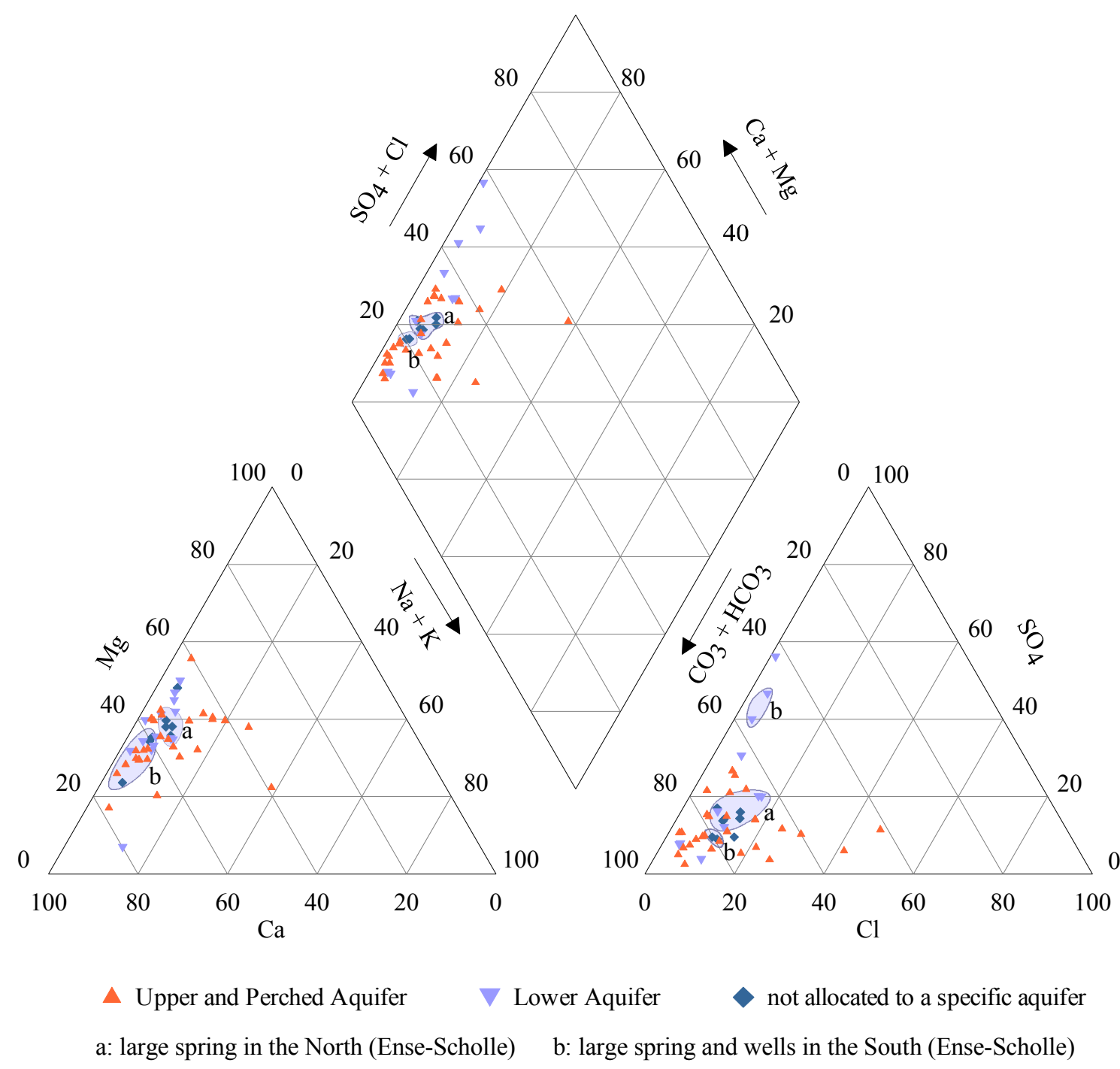

Fig. 4.5: Composition of major ions as Piper plot demonstrated with the samples of August 2010, similar results are obtained with the data from the other sampling campaigns

An important tool for the assessment of the sensitivity to pollution of groundwater bodies is the mapping of the protective effect of the groundwater cover (Neukum \& Hötzl 2007). The assessment of the intrinsic vulnerability is normally based on the field capacity of the soil zone, the intensity of groundwater recharge, permeability and thickness of the vadose zone 
as well as by additional features such as the presence of perched aquifers. Especially in karst systems, a reliable classification of intrinsic vulnerability for predicting raw water qualities relies on the sound knowledge of zones of rapid infiltration as well as on areas of low infiltration rates within the vadose zone (COST 2003). The study area exhibits a wide range of thicknesses of the vadose zone as well as types of bedrock materials determining the intrinsic vulnerability and, consequently, the occurrence of OMPs. Independent of the actual OMP source, the large number and the high concentrations of OMPs detected in an observation well (Reh et al. 2013) suggests rapid influx into the Lower Aquifer and, therefore, a high vulnerability of the groundwater resources south of the city (Fig. 4.4). Here, a river with leakage to groundwater dries out during low flow periods in a carbonate area of the Lower Aquifer. A wastewater sewer and an old waste disposal site are possible additional sources of OMPs. Another old waste disposal site, identified as source of OMPs, is situated in the hydraulically low conductive sandstone and the clay formations of the upper Zechstein (z4$\mathrm{zFb}$ ) northwest of the town. Here, the Perched Aquifer is developed in the embedded conglomerates and in thin carbonate layers. Close to the waste disposal site, the concentrations of paracetamol, phenazone, clofibric acid, primidone, triazines, triazoles and mecoprop are present at elevated concentrations (113-35,000 $\mathrm{ng} \mathrm{L}^{-1}$, Fig. 4.6). With the exception of phenazone and mecoprop the characteristic OMPs were only detected in the close vicinity of the waste disposal. In the spring in the North, mainly fed by the carbonate aquifers, these typical OMPs of the waste disposal are also absent, indicating attenuation potential in combination with dilution by mixing with groundwater from the carbonate aquifers. Consequently, the low conductive sediments in combination with the perched aquifer may slow down vertical transport. This approach is consistent with the assumed low grade of vulnerability regarding the Lower Aquifer. An even lower degree of vulnerability is expected in the East of the study area (Meineringhausen-Scholle). Here, the Zechstein formations are partly covered by more than $100 \mathrm{~m}$ thick formations of the Lower Buntsandstein mainly consisting of low conductive sandstones. Despite the lower population density compared to the North of the Ense-Scholle the release of OMPs is demonstrated by up to 25 different compounds in river water including relatively high concentrations of triazoles (up to $410 \mathrm{ng} \mathrm{L}^{-1} \mathrm{BT}$ and $270 \mathrm{ng} \mathrm{L}^{-1} \mathrm{TT}$ ). However, OMPs are hardly present at all in groundwater samples of the MeineringhausenScholle. Mainly triazines at low concentrations, probably infiltrated at least two decades ago, were detected. Therefore, a long travel time is required to reach the carbonate aquifers confirming the already assumed low vulnerability of groundwater in this area. 


\subsubsection{Detection and characterization of contaminant sources}

The identification of contaminant sources is essential for an effective protection of groundwater resources (EU 2000). Point sources of contaminants are usually investigated by standard analytical methods, e.g. GC-MS, HPLC or ICP-MS, mostly for the detection of hydrocarbons (fuel oil, diesel), aromatic compounds (benzene, polyaromatic hydrocarbons, polychlorinated biphenyls, chlorinated solvents, or heavy metals (e.g. Jensen et al. 1999, Rodenburg et al. 2010, Wiedemeier et al. 1999, Wang et al. 1999). Typical contaminations in the study area are CHCs, released from an old waste disposal and an industrial site, both situated in the catchment area of the spring in the North. CHCs had a wide application as solvents for oils and grease in industrial processes as well as in minor application such as dry cleaning between the 1950s and 1990s. Consequently, large amounts leaked into the subsurface from mainly point sources with eventually overlapping contaminant plumes (e.g. McCarthy \& Johnson 1993). An unambiguous allocation of such plumes to a discrete source or the distinction of transport pathways is therefore a problem. This problem is even exacerbated in karst systems, drained by preferential conduit flow paths, collecting water of large areas. The large spring in the North is influenced by the $\mathrm{CHC}$ tetrachloroethene $\left(4-5 \mu \mathrm{g} \mathrm{L}^{-1}\right)$. Possible sources within the catchment area are a contaminated industrial site with concentrations of CHCs higher than $1000 \mu \mathrm{g} \mathrm{L}^{-1}$ (sum of 9 detected chlorinated ethenes, ethanes and methanes) and with lower concentrations an old waste disposal site (34-58 $\mu \mathrm{g} \mathrm{L}^{-1}$ sum of 3 detected chlorinated ethenes and ethanes). Further detections are limited to only two compounds (tetrachloroethene and 1,1,1-trichloroethane) in a small spring in the North (up to $\left.22 \mu \mathrm{g} \mathrm{L}^{-1}\right)$ and with lowest concentrations $\left(<2 \mu \mathrm{g} \mathrm{L}{ }^{-1}\right)$ in two wells east of the Westheimer Abbruch in the Meineringhausen-Scholle. CHC plumes were not identified, due to the low detection frequency of CHC's, the large distances between sampling points, and the low number of analyzed compounds in relation to their wide application. Consequently, the $\mathrm{CHCs}$ in the large spring in the North and in the Meineringhausen-Scholle may originate from the two known sources as well as from any other not identified location. The larger variety of the different OMPs may provide more information about transport pathways originating from point sources. In the surrounding area of the old waste disposal site a large number of observation wells, developed in the Upper Aquifer and in the Perched Aquifer, provide locally detailed information about the spatial distribution of OMPs. In contrast to the occurrence of $\mathrm{CHC}$ the combination of paracetamol, phenazone, primidone and mecoprop is a characteristic "fingerprint" of the disused waste disposal, because these OMPs were infrequently detected in other parts of the study area. Observed concentrations of phenazone 
are 10 to 100 times higher north than immediately south of it (s. Fig. 4.6). The impact of the waste disposal on the groundwater body can be estimated from the next observation point in northeast direction, which lies approximately $1.9 \mathrm{~km}$ away but still exhibits 3-5 $\mathrm{ng} \mathrm{L}^{-1}$ phenazone. In south direction, phenazone and also mecoprop were detected at a distance of up to $0.8 \mathrm{~km}$. These transport pathways, in north as well as in south direction, are consistent with the observed groundwater gradients and refer also to a locally deviation from the mainly north oriented groundwater flow direction within the Upper Aquifer and in the Perched Aquifer. Further, this result goes beyond the possible interpretations of the distribution of CHCs.

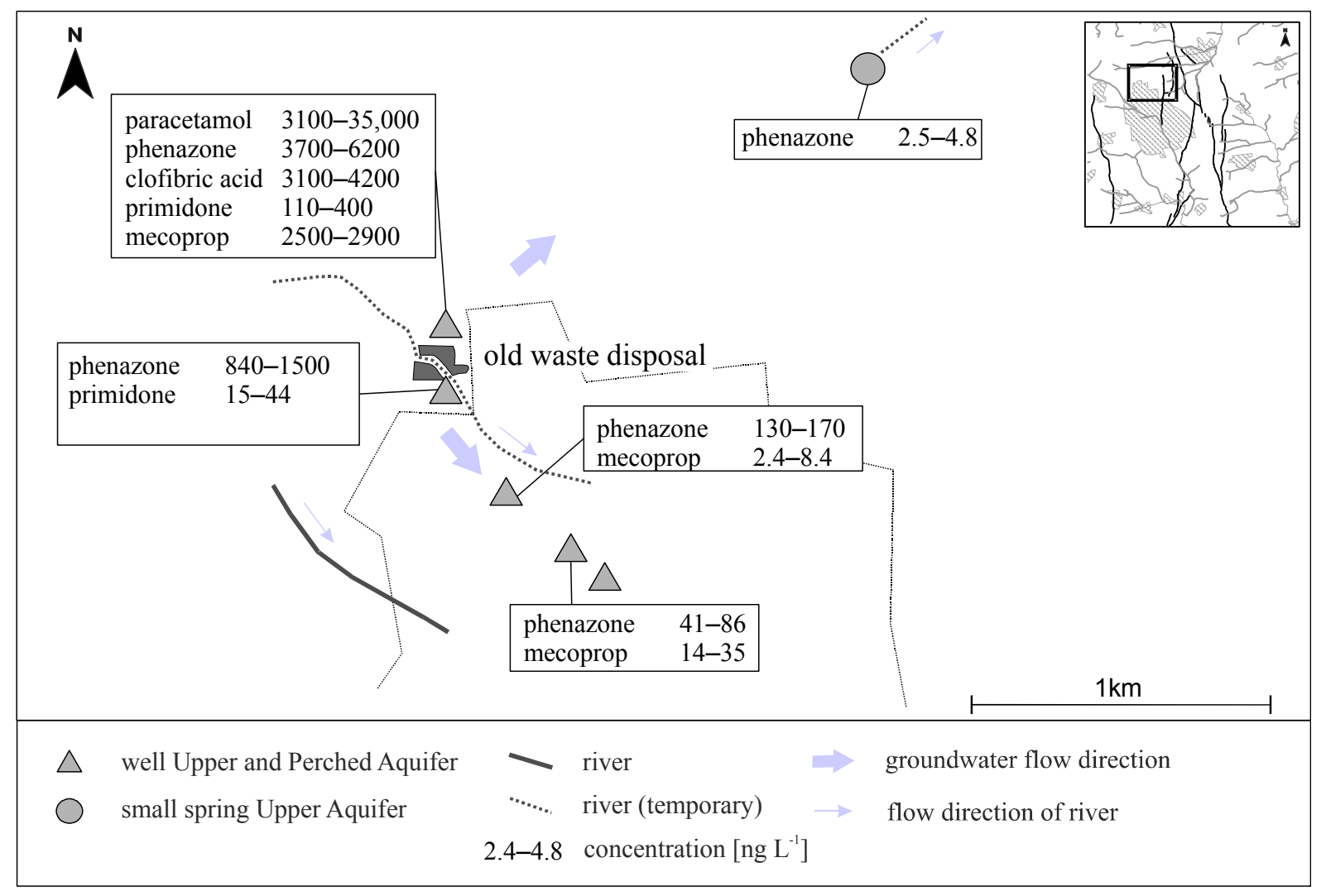

Fig. 4.6: Spatial extension of OMP concentrations originating from a disused waste disposal

To predict the environmental fate of contaminants, e.g. CHCs, the characterization of the redox system plays an important role (e. g. Bouwer \& McCarty 1983). A reliable determination of conventional redox-sensitive parameters, such as dissolved oxygen, nitrate, or free carbon dioxide, requires careful field measurements. Because the persistence of some OMPs in the subsurface depends also on the redox conditions (Banzhaf et al. 2012), OMPs could be useful additional indicators of the redox medium. Phenazone for example is believed to be 
potentially biodegradable under aerobic conditions (Reddersen et al. 2002, Zuehlke et al. 2007). In the study area the persistence of phenazone for more than three decades was indicated by the known operation time of the old waste disposal ending in the 1970ies. In relation to other OMPs (paracetamol, primidone, mecoprop) released from the same disused waste disposal and possible further nearby old waste disposals, phenazone is transported over the longest distance (Fig. 4.6). The mobility of phenazone, paracetamol, and primidone in groundwater is assumed to be high regarding a log $\mathrm{K}_{\mathrm{ow}}<1$ (Hebig et al. 2014). The same also applies to mecoprop, occurring as an anion (Schaffer \& Licha 2014). Hence, the selective attenuation of these OMPs may an indicator for redox conditions and refers to a higher persistence of phenazone in relation to paracetamol, primidone, and mecoprop under reducing conditions. Accordingly, in the surrounding of the waste disposal nitrate reducing conditions were observed, which may inhibit the degradation of phenazone.

\subsection{Conclusions}

OMPs were detected in the investigated complex karst system and could be quantified in groundwater predominantly of the Ense-Scholle. The spatial distribution of persistent compounds shows patterns with contrasts in composition or concentrations. The seasonal distribution was identified to have a low fluctuation within a two years study and enables to reproduce the distribution patterns. Therefore, OMPs were evaluated as potential indicators for the investigation of flow and transport in a complex karst system on catchment scale. The wide variety of analyzed OMPs opens also the possibility to apply several indicators for the same problem and therefore, together with geological-geometric data, contributes to the reduction of ambiguity in the interpretation.

The application of OMPs further supports the development of a consistent conceptual hydrogeological model if OMP information is jointly interpreted with the geological structure, major ion chemistry, and the distribution of other anthropogenic contaminants. In particular, clear contrasts in the spatial distribution are suitable indications for flow in aquifer systems. In principle, low concentrations of a few $\mathrm{ng} \mathrm{L}^{-1}$ can be sufficient to produce such a contrast, since interpretations of OMP patterns are not superimposed by any geogenic background concentration. In the application of OMPs as indicators, the level of their concentrations is therefore less important than appropriate conditions for discharge, such as waste disposals, leakages from sewer systems in urban areas, and zones of elevated vulnerability. Decreasing detection limits would even enhance the possibilities of indications. 
The presented study has shown that the spatial variation of the number of OMPs and the spatial distribution of triazoles may be applied for the delineation of catchment areas. Triazines and point-source OMPs can be employed in vulnerability mapping. These tasks provide solutions for the most important problems in the designation of protection zones, which in karst systems otherwise require large effort.

Due to the different release (point sources vs. diffuse release) and use of OMPs in the catchments, a dependence of their distribution on landuse can be expected. In addition, geological conditions, such as covering layers or perched aquifers, affect the transport of OMPs. Hereby, the broad spectrum of OMPs provides a wide choice of potential indicators to give answers even in a wide range of geological and hydrogeological settings. However, which OMPs are most suitable must be determined from case to case.

The compound atrazine and selected metabolites can be detected even two decades after the large application of atrazine has ceased. Their occurrence contains information on the longterm dispersal behavior. Hydraulic properties of aquitards and fault zones can be derived from triazine spatial distributions, in our case even under limited hydraulic connections. Because of the long travel time of approximately two decades, such information cannot be obtained from artificial tracer experiments.

Point-source OMPs with a low tendency to biodegrade are also characterized by a low variability in their distribution, even though with less spatial extension compared to widely released compounds. They were successfully applied in a qualitative identification of point sources, in the determination of their impact on the groundwater body, and consequently, for the determination of local groundwater flow directions.

\subsection{Acknowledgements}

This work was funded by the Hessisches Landesamt für Umwelt und Geologie HLUG, Wiesbaden, as well as Energie Waldeck-Frankenberg EWF GmbH, Korbach. Therefore, we would like to thank Inga Schlösser-Kluger and Margret Jäger-Wunderer (HLUG) as well as Stefan Schaller and Friedrich Wilke (EWF) for providing the funds. Additional thanks are due to the members of Regierungspräsidium Kassel for supplying long term discharge measurements and archived materials. We would express our gratitude to the Federal Ministry of Education and Research promotional reference number 02WRS1277A, AGRO “Risikomanagement von Spurenstoffen und Krankheitserregern in ländlichen Gebieten". 


\subsection{References}

Adams, C.D., Thurman, E.M.: Formation and transport of deethylatrazine in the soil and vadose zone. J Environ. Qual. 20, 540-547(1991)

Banzhaf, S., Nödler, K., Licha, T., Krein, A., Scheytt, T.: Redox-sensitivity and mobility of selected pharmaceutical compounds in a low flow column experiment. Sci. Total Environ. 438, 113-121 (2012)

Bouwer, E.J., McCarty, P.L.: Transformations of 1- and 2-carbon halogenated aliphatic organic compounds under the methanogenic conditions. Appl. Environ. Microbiol. 45, 1286-1294 (1983)

Clara, M., Strenn, B., Kreuzinger, N.: Carbamazepine as a possible anthropogenic marker in the aquatic environment: investigations on the behaviour of carbamazepine in wastewater treatment and during groundwater infiltration. Water Res. 38, 947-954 (2004)

COST ACTION 620: Vulnerability and Risk Mapping for the Protection of Carbonate (Karst) Aquifers. Final Report. 320 S.; EC, Research DG, Bruxelles (2003)

Daneshvar, A., Svanfelt, J., Kronberg, L., Weyhenmeyer, G.A.: Winter accumulation of acidic pharmaceuticals in a Swedish river. Environ. Sci. Pollut. R. 17, 908-916 (2010)

Estévez, E., del Carmen Cabrera, M., Molina-Díaz, A., Robles-Molina, J., del Pino Palacios-Díaz, M.: Screening of emerging contaminants and priority substances (2008/105/EC) in reclaimed water for irrigation and groundwater in a volcanic aquifer (Gran Canaria, Canary Islands, Spain). Sci. Total Environ. 433, 538-546 (2012)

EU: Directive 2000/60/EC of the European Parliament and of the Council of 23 October 2000, establishing a framework for Community action in the field of water policy. (2000)

EU: Directive 2004/42/CE of the European Parliament and of the Council of 21 April 2004 on the limitation of emissions of volatile organic compounds due to the use of organic solvents in certain paints and varnishes and vehicle refinishing products and amending Directive 1999/13/EC. (2004)

Field, M.S., Pinsky, P.F.: A two-region nonequilibrium model for solute transport in solution conduits in karstic aquifers. J. Contam. Hydrol. 44, 329-351 (2000) 
Ford, D.C., Williams, P.: Karst Hydrogeology and Geomorphology. 562 p.; John Wiley \& Sons Ltd., West Sussex (2007)

Furtak H., Langguth, H.R.: Zur hydrochemischen Kennzeichnung von Grundwässern und Grundwassertypen mittels Kennzahlen. Mem. IAH-Congress 1965 VII, 86-96 (1967)

Geyer, T., Birk, S., Reimann, T., Dörfliger, N., Sauter, M.: Differentiated characterization of karst aquifers: some contributions. Carbonate Evaporite 28, 41-46 (2013)

Giger, W., Schaffner, C., Kohler, H.-P.E.: Benzotriazole and tolyltriazole as aquatic contaminants. 1. Input and occurrence in rivers and lakes. Environ. Sci. Technol. 40, 7186-7192 (2006)

Goldscheider, N.: Tracer tests in karst hydrogeology and speleology. International Journal of Speleology, 37, 27-40 (2008)

Hart, D.S., Davis, L.C., Erickson, L.E., Callender, T.M.: Sorption and partitioning parameters of benzotriazole compounds. Microchem, J. 77, 9-17 (2004)

Hebig, K.H., Nödler, K., Licha, T. Scheytt, T.J.: Impact of materials used in lab and field experiments on the recovery of organic micropollutants. Sci. Total Environ. 473-474, 125-131 (2014)

Heinz, B., Birk, S., Liedl, R., Geyer, T., Straub, K.L., Andresen, J., Bester, K., Kappler, A.: Water quality deterioration at a karst spring (Gallusquelle, Germany) due to combined sewer overflow: Evidence of bacterial and micro-pollutant contamination. Environ. Geol. 57, 797-808 (2009)

Hillebrand, O., Musallam, S., Scherer, L., Nödler, K., Licha, T.: The challenge of sample-stabilisation in the era of multi-residue analytical methods: A practical guideline for the stabilisation of 46 organic micropollutants in aqueous samples. Sci. Total Environ. 454-455, 289298 (2013)

Hillebrand, O., Nödler, K., Geyer, T., Licha, T.: Investigating the dynamics of two herbicides at a karst spring in Germany: Consequences for sustainable raw water management. Sci. Total Environ. 482-483, 193-200 (2014) 
Hillebrand, O., Nödler, K., Licha, T., Sauter, M., Geyer, T.: Caffeine as an indicator for the quantification of untreated wastewater in karst systems. Water Res. 46, 395-402 (2012b)

Hillebrand, O., Nödler, K., Licha, T., Sauter, M., Geyer, T.: Identification of the attenuation potential of a karst aquifer by an artificial dualtracer experiment with caffeine. Water Res. 46, 5381-5388 (2012a)

Hölting. B., Matthess, G.: Ein Sporentriftversuch im Zechstein von Korbach/ Hessen. Notizbl. HlfB 91, 237-255 (1963)

Jensen, D.L., Ledin, A., Christensen, T.H.: Speciation of heavy metals in landfill-leachate polluted groundwater. Water Res. 33, 2642-2650 (1999)

Käss, W., Behrens, H: Tracing technique in geohydrology. 581 p.; Balkema, Rotterdam (1998)

Kolpin, D.W., Furlong, E.T., Meyer, M.T., Thurman, E.M., Zaugg, S.D., Barber, L.B., Buxton. H.T.: Pharmaceuticals, hormones, and other organic wastewater contaminants in U.S. streams, 1999-2000: A national reconnaissance. Environ. Sci. Technol. 36, 1202-1211 (2002)

Kulick, J.: Erläuterungen zur Geologischen Karte von Hessen 1:25.000, Blatt 4719 Korbach. 2. Aufl., 272 p.; Wiesbaden (1997)

Licha, T., Niedbala, A., Bozau, E., Geyer, T.: An assessment of selected properties of the fluorescent tracer, Tinopal CBS-X related to conservative behavior, and suggested improvements. J. Hydrol. 484, 38-44 (2013)

Massmann, G., Dunnbier, U., Heberer, T., Taute, T.: Behaviour and redox sensitivity of pharmaceutical residues during bank filtration -Investigation of residues of phenazone -type analgesics. Chemosphere 71, 1476-1485 (2008)

McCarthy, K.A., Johnson, R.L.: Transport of volatile organic compounds across the capillary fringe. Water Resour. Res. 29, 1675-1683 (1993)

Menning, M., Hendrich, A.: Stratigraphische Tabelle von Deutschland 2002.; Tafel 96x130 cm oder Falt-Tafel A4. Deutsche Stratigraphische Kommission, Potsdam (Geoforschungszentrum), Frankfurt a. M. (Forsch.-Inst. Senckenberg) (2002) 
Metcalfe, C.D., Beddows ,P.A., Bouchot, G.G., Metcalfe, T.L., Li, H., Van Lavieren, H.: Contaminants in the coastal karst aquifer system along the Caribbean coast of the Yucatan Peninsula, Mexico. Environ. Pollut. 159, 991-997 (2011)

Morasch, B.: Occurrence and dynamics of micropollutants in a karst aquifer. Environ. Pollut. 173, 133-137 (2013)

Müller, B., Scheytt, T., Asbrand, M., Mross de Casas, A.: Pharmaceuticals as indictors of sewage-influenced groundwater. Hydrogeol. J. 20, 1117-1129 (2012)

Neukum, C., Hötzl, H.: Standardization of vulnerability maps. Environ. Geol. 51, 689-694 (2007)

Nödler, K., Licha, T., Bester, K., Sauter, M.: Development of a multi-residue analytical method, based on liquid chromatography-tandem mass spectrometry, for the simultaneous determination of 46 micro-contaminants in aqueous samples. J. Chromatogr. A 1217, 6511-6521 (2010)

Nödler, K., Licha, T., Fischer, S., Wagner, B., Sauter, M.: A case study on the correlation of micro-contaminants and potassium in the Leine River (Germany). Appl. Geochem. 26, 2172-2180 (2011)

Reddersen, K., Heberer, T., Dünnebier, U.: Identification and significance of phenazone drugs and their metabolites in ground- and drinking water. Chemosphere 49, 539-544 (2002)

Reh R., Licha T., Geyer T., Nödler, K., Sauter M.: Occurrence and spatial distribution of organic micro-pollutants in a complex hydrogeological karst system during low flow and high flow periods, results of a two-year study. Sci. Total Environ. 443, 438-445 (2013)

Rodenburg, L.A., Du, S., Fennel, D.E., Cavallo, G.J.: Evidence for widespread dechlorination of polychlorinated biphenyls in groundwater, landfills, and wastewater collection systems. Environ. Sci. Technol. 44, 7534-7540(2010)

Sacher, F., Ehmann, M., Gabriel, S., Graf, C., Brauch, H.J.: Pharmaceutical residues in the river Rhine - results of a one-decade monitoring programme. J. Environ. Monit. 10, 664670 (2008) 
Sauter, M.: Quantification and forecasting of regional groundwater flow and transport in a karst aquifer (Gallusquelle, Malm, SW. Germany). 150 S.; Tübinger Geowissenschaftliche Arbeiten C13, Tübingen (1992)

Schaffer, M., Licha, T.: Review: A guideline for the identification of environmentally relevant, ionizable organic molecule species. Chemosphere 103, 12-25 (2014)

Schraft, A., Fritsche, J. G., Hemfler, M., Mittelbach, G., Rambow, D., Tangermann, T.: Die hydrogeologischen Einheiten Nordhessens, ihre Grundwasserneubildung und ihr nutzbares Grundwasserdargebot (Ldkrs. Waldeck-Frankenberg, Kassel, Schwalm-Eder, WerraMeißner, Hersfeld-Rotenburg, Fulda und Stadt Kassel). Geol. Jb. Hessen 129, 27-53 (2002)

Schwarzenbach, R.P., Escher, B.I., Fenner, K., Hofstetter, T.B., Johnson, C.A., von Gunten, U., Wehrli, B.: The challenge of micropollutants in aquatic systems. Science 313, 1072-1077 (2006)

Stuart, M.E., Lapworth, D.J., Thomas, J., Edwards. L.: Fingerprinting groundwater pollution in catchments with contrasting contaminant sources using microorganic compounds. Sci. Total Environ. 468-469, 564-577 (2014)

Tappe, W., Groeneweg, J., Jantsch, B.: Diffuse atrazine pollution in German aquifers. Biodegradation 13, 3-10 (2002)

Thurman, E.M., Fallon, J.D.: The Deethylatrazine/Atrazine ratio as an indicator of the onset of the spring flush of herbicides into surface water of the midwestern United States. Int. J. Environ. An. Ch. 65, 203-214 (1996)

Wang, Z.D., Fingas, M., Page, D.S.: Oil spill identification. J. Chromatogr. A 843, 369-411 (1999)

WHO: Guidelines for drinking-water quality, 4th edition. http://whqlibdoc.who.int/publications/2011/9789241548151_eng.pdf (2011). Accesssed 21 November 2013

Wiedemeier, T.H., Rifai, H.S., Newell, C.J., Wilson, J.T.: Natural attenuation of fuels and chlorinated solvents in the subsurface. 632 p.; Wiley, New York (1999)

Wolf, L., Held, I., Eiswirth, M., Hötzl, H.: Impact of leaky sewers on groundwater quality. Acta hydrochim. hydrobiol. 32, 361-373 (2004) 
Wolschke, H., Zhiong, X., Möller, A., Sturm. R., Ebinghaus, R.: Occurrence, distribution and fluxes of benzotriazoles along the German large river basins into the North Sea. Water Res. 45, 6259-6266 (2011)

Worthington, S.R.H.: A comprehensive strategy for understanding flow in carbonate aquifer. Speleogenesis and Evolution of Karst Aquifers 1(1) (2003)

Zuehlke, S., Duennbier, U., Heberer, T.: Investigation of the behavior and metabolism of pharmaceutical residues during purification of contaminated ground water used for drinking water supply. Chemosphere 69, 1673-1680 (2007) 



\section{Charakterisierung zweier Karstsysteme mit Hilfe organischer Spurenstoffe}

Application of organic micro-pollutants for the characterisation of two karst systems

Roland Reh ${ }^{\mathrm{a}}$, Olav Hillebrand ${ }^{\mathrm{a}}$, Tobias Geyer ${ }^{\mathrm{a}, \mathrm{b}}$, Karsten Nödler ${ }^{\mathrm{a}}$, Tobias Licha ${ }^{\mathrm{a}}$, Martin Sauter ${ }^{\mathrm{a}}$

Zitat:

Reh, R., Hillebrand, O., Geyer, T., Nödler, K., Licha, T., Sauter, M.: Charakterisierung zweier Karstsysteme mit Hilfe organischer Spurenstoffe. Grundwasser 19,

251-262 (2014)

http://dx.doi.org/10.1007/s00767-014-0264-6

(C) Springer-Verlag Berlin Heidelberg 2014

Mit freundlicher Genehmigung der Springer Science+Business Media

${ }^{a}$ Universität Göttingen, Geowissenschaftliches Zentrum, Angewandte Geologie,

Goldschmidtstraße 3, 37077 Göttingen, Deutschland

${ }^{b)}$ Landesamt für Geologie, Rohstoffe und Bergbau (LGRB) im Regierungspräsidium

Freiburg, Albertstraße 5, 79104 Freiburg im Breisgau, Deutschland 


\section{Kurzfassung}

In der vorliegenden Arbeit wird das Auftreten organischer Spurenstoffe in zwei hinsichtlich der geologischen Verhältnisse und der Flächennutzung unterschiedlichen Karstgebieten diskutiert.

In einem urban geprägten, moderat verkarsteten Quelleinzugsgebiet mit einer hohen Anzahl lokaler Eintragsquellen (beispielsweise Altlasten, Kanalleckagen) ist gegenüber einem landwirtschaftlich dominierten Gebiet das Spektrum der gefundenen Stoffe breiter. Die Konzentrationen der meisten dieser Stoffe nehmen auf der Fließstrecke zu den Quellaustritten bis unter die Bestimmungsgrenzen ab und können daher nur über ein flächendeckendes Messstellennetz erfasst werden. In den Quellen finden sich vorwiegend Stoffe, die entweder durch eine weite rezente Anwendungsverbreitung oder hohe Persistenz charakterisiert sind.

In der Quelle eines stärker verkarsteten und somit höher durchlässigen Grundwasserfließsystems werden zeitlich variierend auch biologisch leicht abbaubare Substanzen detektiert. Aufgrund hoher Fließgeschwindigkeiten erfordern Untersuchungen dieses Fließsystems ein zeitlich hochaufgelöstes Monitoring organischer Spurenstoffe.

Somit haben neben den jeweiligen Stoffeigenschaften auch die hydrogeologischen Systemcharakteristika erheblichen Einfluss auf das Spektrum der nachweisbaren Stoffe. 


\section{Abstract}

In the presented work the occurrence of organic micro-pollutants is discussed for two karst areas with different geological characteristics and land-use patterns.

Within an urban moderately karstified catchment with a large number of local input sources (e.g. contaminated sites, sewer leakages), a greater variety of micro-pollutants is found compared to a rural area. Mostly the concentrations of these compounds decrease below quantification limits before emerging at the spring and can therefore only be detected by a comprehensive network of sampling points. Springs show the presence of micro-pollutants, either recently applied in large amounts or characterized by high persistence.

At the outlet of a highly karstified and consequently highly permeable aquifer readily biodegradable compounds are detected. Due to high groundwater flow velocities investigations of micro-pollutants require a highly resolved monitoring.

Therefore, beside substance properties, also hydrogeological characteristics affect the spectrum of detectable micro-pollutants. 


\subsection{Einleitung}

Komplexe Fließsysteme wie Kluftgrundwasserleiter mit mehreren übereinanderliegenden Stockwerken oder Karbonatgesteine mit starker Verkarstung sind mit herkömmlichen hydrogeologischen Methoden schwierig zu erkunden. Probleme bei der Charakterisierung verursachen vor allem lokale Heterogenitäten, die als präferentielle Fließwege fungieren (Atkinson 1977, Einsiedl 2005, Geyer et al. 2013).

Aufgrund ihrer hohen Ergiebigkeit stellen Karstgrundwasserleiter potentielle und tatsächliche Trinkwasservorkommen dar, zu deren Nutzung und Schutz Kenntnisse über grundlegende Eigenschaften wie Einzugsgebietsgröße und Fließverhalten erforderlich sind (Ford \& Williams 2007). Die Weltgesundheitsorganisation hat in ihren Richtlinien zur Trinkwasserqualität ausdrücklich auf die Vorteile und Notwendigkeit eines effektiven Einzugsgebietsmanagements hingewiesen (WHO 2011). Hierzu zählen sowohl das Verständnis des Grundwasserleitersystems als auch die Identifikation möglicher Schadstoffquellen, welche die Rohwasserqualität beeinflussen können. Die Anwendung konventioneller Methoden zur Systemcharakterisierung auf der Einzugsgebietsskala, wie beispielsweise künstliche Markierungsversuche, erfordert in weitgehend unbekannten Systemen einen hohen Aufwand. Daher könnten Methoden hilfreich sein, die indikativ Informationen über großskalige Fließsysteme liefern.

Seit Beginn der Industrialisierung entlässt der Mensch eine Vielzahl unterschiedlicher Stoffe in die Umwelt. Dazu gehören vor allem organische Substanzen, wie industriell verwendete Chemikalien, Arzneimittel, Pflanzenschutzmittel aber auch Alltagsprodukte wie Koffein (Kolpin et al. 2002, Schwarzenbach et al. 2006). In den letzten rund 20 Jahren wurden die analytischen Labormethoden zum Nachweis dieser Stoffe entwickelt und verfeinert, mit denen gezeigt werden konnte, dass eine große Anzahl dieser Stoffe auch in aquatischen Systemen nachweisbar ist (Ternes 2007). Nachdem organische Spurenstoffe zuerst in Oberflächengewässern und dann in Porengrundwassersystemen, vor allem Uferfiltraten, nachgewiesen wurden (Heberer et al. 2004, Massmann et al. 2008, Sacher et al. 2008), zeigten neuere Studien auch das Vorkommen in komplexen Kluft- und Karstgrundwasserleitern (Estévez et al. 2012, Morasch 2013, Hillebrand et al. 2012a, Reh et al. 2013). Die beiden letztgenannten Studien wiesen darauf hin, dass die Eigenschaften der Fließsysteme wesentlichen Einfluss auf die beobachtete Stoffpalette als auch auf deren zeitliche und räumliche Verteilung haben, das heißt, dass organische Spurenstoffe potentielle Indikatoren für Fließ- und Transportpro- 
zesse im Untergrund darstellen, wenn sie in signifikanten Mengen eingetragen wurden Da bisherige Fallbeispiele (zum Beispiel Metcalfe et al. 2011, Hillebrand et al. 2012a, Reh et al. 2013) jeweils für ein spezifisches Untersuchungsgebiet gelten, konnte nicht gezeigt werden, ob die jeweilige Indikatoreigenschaft verallgemeinerbar auf andere Gebiete übertragen werden kann. Systemübergreifende Vergleiche sind nach Kenntnis der Autoren noch nicht publiziert. Mit der vorliegenden Arbeit soll der indikative Charakter organischer Spurenstoffe hinsichtlich der anthropogenen Beeinflussung und grundlegender hydrodynamischer Eigenschaften in zwei Karstsystemen mit unterschiedlicher geografischer Lage, Landnutzung und auch verschiedenen geologischen Eigenschaften miteinander verglichen werden. Beide Untersuchungsgebiete liegen in Deutschland, zum einen im Nordwesten Hessens, zum anderen auf der Schwäbischen Alb. Der Vergleich beruht auf neuen Messwerten und teilveröffentlichten Datensätzen (Hillebrand et al. 2012a, Hillebrand et al. 2014, Reh et al. 2013).

\subsection{Die Untersuchungsgebiete}

Im Nordwesten Hessens hat sich am Ostrand des Rheinischen Schiefergebirges in den Karbonatformationen des Zechsteins (z1-z3) ein Grundwasserleitersystem mit mehreren Stockwerken ausgebildet (s. Abb. 5.1). Die drei Grundwasserleiter der Karbonatformationen mit Mächtigkeiten zwischen 0,5 und $40 \mathrm{~m}$ sind moderat verkarstet und teilweise mit Klastiten der höheren Zechsteinformationen und des Unteren Buntsandsteins überdeckt (Kulick 1997). Die beiden wasserwirtschaftlich genutzten Grundwasserleiter befinden sich in den Karbonaten der Werra-Formation (unterer Grundwasserleiter) und der Leine-Formation. Letztere werden mit den geringleitenden Klastiten der oberen Zechsteinformationen (z4-z7, Frankenberg-Formation) in diesem Artikel als oberes Grundwasserstockwerk zusammengefasst. Das Untersuchungsgebiet wird nach den Geologischen Struktureinheiten als „Waldecker Scholle“ (HLUG 2008) bezeichnet und umfasst die in einer Plateaulage aneinander grenzenden drei Quelleinzugsgebiete der Flüsse Twiste, Itter und Werbe. Der natürliche Austritt des Grundwassers erfolgt über Quellgruppen am Ursprung der Flüsse. Der Westheimer Abbruch trennt als wichtigstes tektonisches Störungselement mit Versatzbeträgen von teilweise mehr als $100 \mathrm{~m}$ die Ense-Scholle im Westen von der Meineringhausen-Scholle im Osten (Schraft et al. 2002). Die Landnutzung umfasst sowohl ein Stadtgebiet mit Industriestandorten und Altlasten als auch Land- und Forstwirtschaft in der Umgebung des Stadtgebiets. 


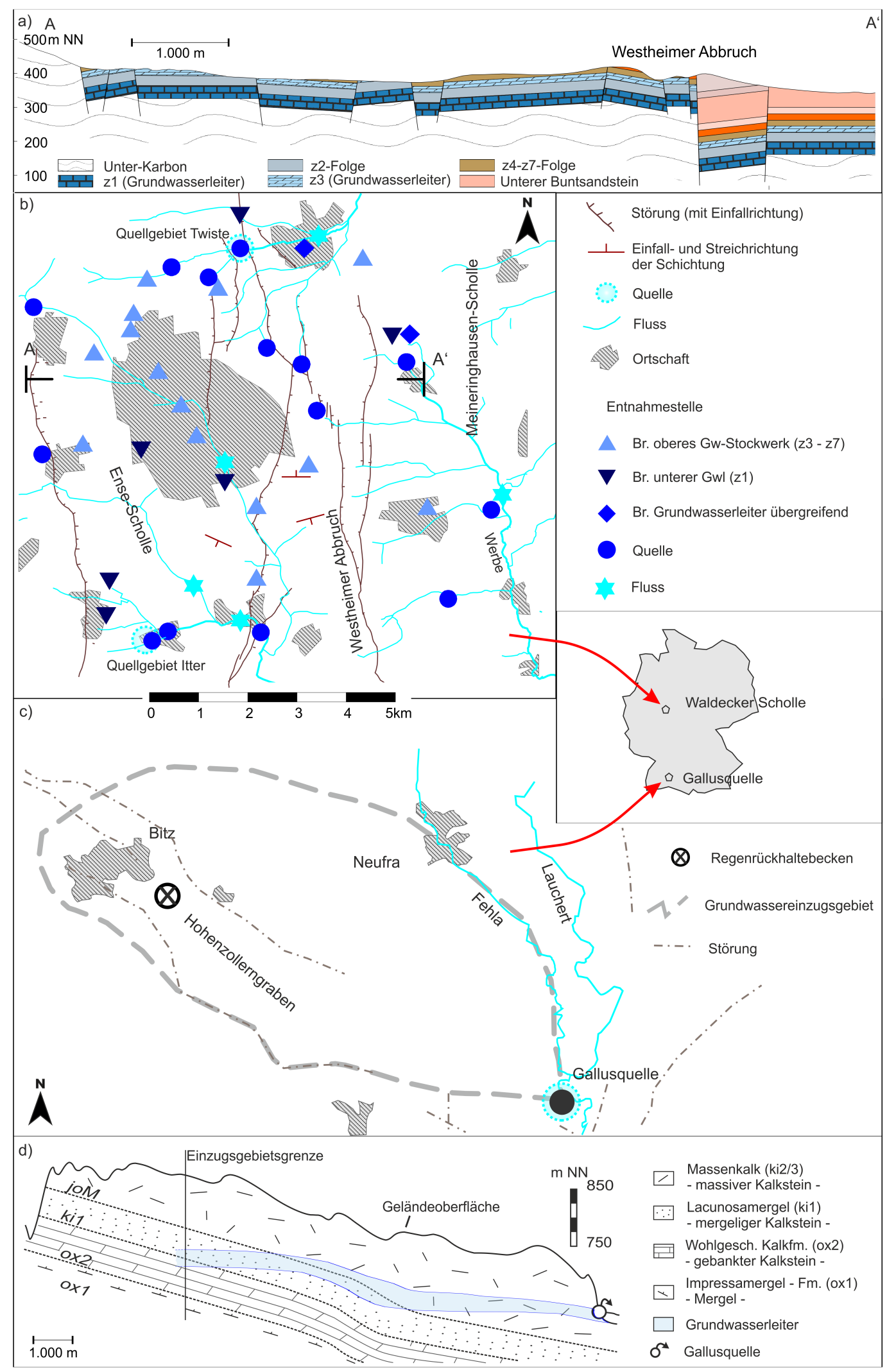

Abb. 5.1: a) Geologischer Profilschnitt der Waldecker Scholle nach Käding (2005) und Kulick (1997) b) Übersichtskarte der Waldecker Scholle mit Lage der Probennahmestellen c) Übersichtskarte des Einzugsgebiets der Gallusquelle verändert nach Geyer et al. (2007) d) Geologischer Profilschnitt des Einzugsgebiets der Gallusquelle nach Sauter (1992) 
Auf der Schwäbischen Alb wird ein schichtenübergreifender Grundwasserleiter mit einer mittleren Mächtigkeit von 20 m in den Karbonatschichten des Oberen Malm (ki1 bis ki2/3) betrachtet. Die Verkarstung ist hier stärker ausgeprägt als in den Zechsteinschichten Nordhessens und weist mit der Gallusquelle einen typischen Hauptaustritt des vorwiegend landwirtschaftlich genutzten Quelleinzugsgebiets auf. Die Schüttung der Gallusquelle beträgt im Jahresmittel etwa 500 l/s (Geyer et al. 2011) und wird für die Trinkwasserversorgung von rund 40.000 Personen verwendet. Das Fließ- und Transportverhalten im Grundwasserleiter der Gallusquelle wird stark durch ein Grabensystem, den herzynisch streichenden Hohenzollerngraben, beeinflusst, in dessen südöstlicher Fortsetzung die Gallusquelle austritt. Im westlichen Bereich des Einzugsgebietes der Gallusquelle dient ein Regenrückhaltebecken während Starkniederschlägen der Entlastung des Mischabwassersystems. Ein Überlaufen des Beckens und eine darauffolgende zeitlich begrenzte Beeinflussung der Qualität des Quellwassers wurde mehrfach beobachtet (Heinz et al. 2009; Hillebrand et al. 2012a).

Beide Untersuchungsgebiete liegen mit einer Fläche von circa $65 \mathrm{~km}^{2}$ (Waldecker Scholle) und circa $45 \mathrm{~km}^{2}$ (Gallusquelle) im gleichen Größenmaßstab und weisen Quellen mit hoher Schüttung auf, die in Karstsystemen typischerweise große Einzugsgebiete entwässern (White 1969). In der Waldecker Scholle beträgt die aus langjährigen Abflussmessungen der Twiste, Itter und Werbe im Rahmen der Studie berechnete Grundwasserneubildung circa 165 mm/a bei einer mittleren Niederschlagsmenge (1961-1990) von circa 730 mm/a (DWD 2013). Im Einzugsgebiet der Gallusquelle führen mittlere jährliche Niederschläge von circa $900 \mathrm{~mm} z u$ einer höheren Grundwasserneubildung von circa 340 mm/a (Sauter 1992). Unterschiedlich ausgeprägt ist auch die Dynamik von Strömung und Transportverhalten des Grundwassers, die sich in den beobachteten Quellschüttungen widerspiegelt (Abb. 5.2 und 5.3). Während in der Waldecker Scholle die Schüttungsratenquotienten (niedrigste Schüttung QN/ höchste Schüttung QH) der Twiste und Itter in Langzeitmessungen (Twiste seit 2001, Itter seit 1975) zwischen 0,05 (Twiste) und 0,09 (Itter) liegen und die Auslaufkoeffizienten $\alpha$ der Quellen rund $0,01 \mathrm{~d}^{-1}$ betragen, sind der Schüttungsratenquotient der Gallusquelle (etwa 0,019) und deren Auslaufkoeffizient (0,002-0,07 d $\mathrm{d}^{-1}$ ) kleiner (Geyer et al. 2011). Diese Werte zeigen somit eine höhere Varianz der Schüttung der Gallusquelle an. 


\subsection{Methoden}

In der Waldecker Scholle wurden innerhalb von zwei Jahren (2010-2011) vier Beprobungskampagnen an jeweils 30 bis 50 Probenahmestellen, vorwiegend Grundwassermessstellen, Quellen und Förderbrunnen (s. Abb. 5.1), jeweils im Frühjahr und Spätsommer/ Herbst durchgeführt (Reh et al. 2013). Damit wurden sowohl Proben innerhalb der Quelleinzugsgebiete als auch an den Quellaustritten gewonnen. Aufgrund des hohen Flurabstands im Einzugsgebiet der Gallusquelle konzentrierte sich die Beprobung ausschließlich auf die Quelle selbst. Dafür wurden hier Proben mit einer hohen zeitlichen Auflösung von bis zu 8 Proben pro Tag entnommen (Beprobungszeitraum 25.02.2010-16.02.2011).

Mittels einer HPLC-MS/MS-Analytik (Nödler et al. 2010; Reh et al. 2013) wurden die Proben der Waldecker Scholle auf 54 verschiedene organische Spurenstoffe untersucht. Die Bestimmungsgrenzen (BG) der verschiedenen Substanzen lagen zwischen 1,2 und $28 \mathrm{ng} / \mathrm{l}$. Die Analytenauswahl umfasst Pestizide, Arzneimittel, Rostschutzmittel sowie auch Alltagsprodukte wie Koffein (s. Anhang, Tab. 5.4). Neben den Ausgangsstoffen wurden zusätzlich einige Metaboliten untersucht. Im Unterschied zu Nödler et al. (2010) und Reh et al. (2013) wurde bei den Wasserproben der Gallusquelle ein höherer Anreicherungsfaktor gewählt, um die hohe Verdünnung des Systems teilweise zu kompensieren, wodurch geringere Nachweisgrenzen (NG) und BG (1,0-22,4 ng/l) erreicht werden konnten. 
Tab. 5.1 Organische Spurenstoffe mit Anwendungsgebieten und Bestimmungsgrenzen, aufgeführt sind Stoffe mit Detektionshäufigkeiten $\geq 10 \%$ quantifizierbarer Konzentrationen (absteigende Sortierung) in den Grundwasserproben beider Untersuchungsgebieten (Angaben zur Waldecker Scholle aus Reh et al. (2013))

\begin{tabular}{llll}
\hline Waldecker Scholle & BG [ng/l] & Gallusquelle & BG [ng/l] \\
\hline Desethylatrazin (PSM-Metabolit) & 1,7 & Desethylatrazin & 1,4 \\
Atrazin (PSM) & 1,4 & Atrazin & 1,1 \\
1H-Benzotriazol (Rostschutzm.) & 4,7 & Koffein & 3,4 \\
Tolyltrizol (Rostschutzm.) & 4,9 & Paraxanthin (Koff.-Metabolit) & 2,6 \\
Desisopropylatrazin (PSM-Metabolit) & 5,6 & Theobromin (Koff.-Metabolit, Stimulanz) & 4,1 \\
Mecoprop (PSM) & 1,2 & Metazachlor (PSM) & 1,4 \\
Terbuthylazin (PSM) & 3,0 & Atenololsäure (Arzneim.-Metabolit) & 3,2 \\
Carbamazepin (Antiepileptikum) & 2,2 & & \\
Diuron (PSM) & 3,3 & & \\
Phenazon (Schmerzmittel) & 2,0 & & \\
Primidon (Antiepileptikum) & 2,7 & & \\
Isoproturon (PSM) & 3,0 & & \\
Metoprolol (Blutdrucksenker) & 4,1 & & \\
\hline
\end{tabular}

$B G$ Bestimmungsgrenze, PSM Pflanzenschutzmittel

\subsection{Ergebnisse und Diskussion}

Die Grundwasserproben aus dem Untersuchungsgebiet Waldecker Scholle zeigten mit einem quantitativen Nachweis von 44 der 54 untersuchten Stoffe insgesamt eine große Vielfalt. Weitere sechs Stoffe konnten in Bächen nachgewiesen werden, die teilweise in Bereichen fehlender Deckschichten direkt in die Karbonatschichten infiltrieren. 13 Stoffe waren in $\geq 10 \%$ der untersuchten Grundwasserproben quantifizierbar (s. Tab. 5.1). Als Eintragspfade konnten flächige Pestizideinträge in ländlichen Bereichen, punktuelle Stoffquellen aus Altlasten sowie Freisetzungen aus dem Kanalsystem identifiziert werden (Reh et al. 2013). Eine Besonderheit ist der oben erwähnte Stoffeintrag über Bäche mit influenten Grundwasserverhältnissen. Die in Tab. 5.1 aufgeführten Stoffe wurden insbesondere im Einzugsgebiet der Twiste im nördlichen Teil des Untersuchungsgebiets Waldecker Scholle eingetragen. Dieses Gebiet ist zudem durch eine relativ hohe Anzahl unterschiedlicher Spurenstoffe charakterisiert.

Gegenüber der Waldecker Scholle ist das gemessene Stoffspektrum an der Gallusquelle deutlich kleiner. Insgesamt wurden 32 verschiedene Komponenten nachgewiesen, von denen lediglich 9 in $\geq 10 \%$ der Proben quantifizierbar waren (Tab. 5.1). Als wesentlicher Grund für 
die unterschiedliche Anzahl der gefundenen Komponenten kann die in der Waldecker Scholle urbane Landnutzung mit einer höheren Vielfalt der eingesetzten Stoffe sowie einer hohen Anzahl möglicher punktueller Eintragsquellen, wie Altlasten und ein Kanalsystem, im Kontrast zum dominierend landwirtschaftlich genutzten Einzugsgebiet der Gallusquelle gesehen werden. Weiterhin ist auf der Schwäbischen Alb durch die deutlich höhere Grundwasserneubildungsrate mit $340 \mathrm{~mm} / \mathrm{a}$ gegenüber $165 \mathrm{~mm} / \mathrm{a}$ in der Waldecker Scholle mit einer stärkeren Verdünnung der eingetragenen Spurenstoffe zu rechnen. Die Quellschüttung der Gallusquelle zeigt zudem eine höhere Dynamik als die Quellschüttungen der Waldecker Scholle, so dass hier von einem höheren Volumenanteil einer Fließkomponente mit kurzer Verweilzeit auszugehen ist.

In beiden Untersuchungsgebieten sind Triazine vorhanden. Das zu den Triazinen gehörende Atrazin (ATR) ist aufgrund seiner relativ hohen Mobilität und Persistenz in Boden und Grundwasser (Jablonowski et al. 2011) seit 1992 durch die Pflanzenschutz-Anwendungsverordnung in Deutschland nicht mehr als Herbizid zugelassen (PflSchAnwV 1992). In der Waldecker Scholle wurden ATR und das Triazin-Abbauprodukt Desethylatrazin (DEA) flächendeckend mit geringer örtlicher Variabilität nachgewiesen. Beide Stoffe sind die am häufigsten nachgewiesenen Komponenten im Untersuchungsgebiet. Charakteristisch für Einträge in urbanen Gebieten ist hier das zusätzliche Auftreten von Desisopropylatrazin (DIA), eines weiteren Triazin-Metaboliten, der punktuell im Einflussbereich einer Altablagerung Konzentrationen bis in den $\mu \mathrm{g} / \mathrm{l}$-Bereich erreicht (Reh et al. 2013). Auch in der Gallusquelle gehören ATR und DEA, deren Konzentrationen in $>80 \%$ der untersuchten Proben oberhalb der BG lagen, zu den am häufigsten vorkommenden Komponenten. Da hier die DIA-Komponente völlig fehlt, ist davon auszugehen, dass die Detektion dieses Metaboliten bevorzugt mit eher punktuellen Einträgen, beispielsweise aus Altablagerungen, in Zusammenhang steht, während bei den im landwirtschaftlichen Bereich typischen flächenhaften Triazin-Einträgen bevorzugt DEA zu beobachten ist. Auch Studien anderer landwirtschaftlich geprägter Untersuchungsgebiete zeigten das bevorzugte Auftreten von DEA bei gleichzeitiger geringer Detektionshäufigkeit von DIA in verkarsteten Grundwasserleitern (Rowden et al. 2001, Baran et al. 2008). Der Vergleich der gemessenen Konzentrationen zeigt, dass in der Waldecker Scholle sowohl die Median- als auch die Maximalkonzentrationen von ATR und DEA höher liegen, als in der Gallusquelle, in der die höhere Grundwasserneubildungsrate und der konzentrierte Abfluss auf eine einzelne Quelle grundsätzlich zu einer stärkeren Verdünnung von Stoffkonzentrationen führen (Tab. 5.2). 
Tab. 5.2 Vergleich der Min.- Median- und Max.-Konzentrationen der Befunde >BG mit Angabe der Detektionshäufigkeit, absteigend sortiert nach Detektionshäufigkeit

\begin{tabular}{|c|c|c|c|c|c|c|c|c|c|c|c|c|c|}
\hline \multicolumn{9}{|l|}{ Waldecker Scholle } & \multicolumn{5}{|l|}{ Gallusquelle } \\
\hline \multirow{2}{*}{$\frac{\text { Stoffname }}{\text { Desethylatrazin }}$} & \multicolumn{4}{|c|}{ Alle Messstellen $(n=163)^{\mathrm{g}}$} & \multicolumn{3}{|c|}{ Quellen $(n=20)$} & & \multicolumn{2}{|l|}{ Stoffname } & \multicolumn{3}{|c|}{$n=283$} \\
\hline & 71 & 2,3 & 18 & 171 & 100 & 8,9 & 27 & 55 & Desethylatrazin & 86 & 1,4 & 2,3 & 5,9 \\
\hline Atrazin & 61 & 1,7 & 7,8 & 86 & 95 & 2,3 & 17 & 35 & Atrazin & 84 & 1,1 & 2,3 & 5,2 \\
\hline 1H-Benzotriazol & 41 & 4,9 & 43 & 2.300 & 48 & 4,9 & 13 & 20 & Koffein & 40 & 3,5 & 8,4 & 86 \\
\hline Tolyltriazol & 31 & 5,2 & 23 & 210 & 52 & 5,4 & 24 & 46 & Paraxanthin & 35 & 2,6 & 5,3 & 46 \\
\hline Desisopropylatrazin & 23 & 6,9 & 22 & 1.800 & 33 & 8,5 & 10 & 22 & Theobromin & 34 & 4,1 & 9,4 & 85 \\
\hline Mecoprop & 17 & 1,2 & 110 & 2.900 & 0 & & & & 1H-Benzotriazol & $23^{\mathrm{e}}$ & 3,8 & 6,0 & 40 \\
\hline Terbuthylazin & $15^{\mathrm{a}}$ & 3,2 & 5,4 & 93 & $17^{\mathrm{c}}$ & 3,3 & 5,7 & 13 & Metazachlor & 17 & 1,4 & 2,5 & 72 \\
\hline Carbamazepin & 13 & 2,4 & 38 & 350 & 0 & & & & Tolyltriazol & $13^{\mathrm{e}}$ & 4,0 & 6,7 & 21 \\
\hline Diuron & 13 & 3,3 & 5,3 & 20 & 52 & 3,3 & 5,7 & 13 & Atenololsäure & $10^{\mathrm{f}}$ & 3,3 & 4,5 & 18 \\
\hline Phenazon & 12 & 2,5 & 150 & 6.200 & 0 & & & & 1-Methylxanthin & 8,8 & 18 & 29 & 199 \\
\hline Primidon & 10 & 3,3 & 15 & 400 & 0 & & & & Iohexol & 6,7 & 18 & 24 & 100 \\
\hline Isoproturon & 10 & 3,3 & 6,4 & 78 & 0 & & & & Iopromid & 4,6 & 14 & 27 & 69 \\
\hline Metoprolol & $10^{\mathrm{b}}$ & 4,9 & 16 & 74 & $0^{\mathrm{d}}$ & & & & $\ldots$ & & & & \\
\hline$\ldots$ & & & & & & & & & 3-Methylxanthin & 2,5 & 23 & 29 & 138 \\
\hline Haloperidol & 7 & 4,1 & 6,5 & 26 & 19 & 4,5 & 6,1 & 6,9 & Iomeprol & 2,1 & 23 & 110 & 270 \\
\hline$\ldots$ & & & & & & & & & Isoproturon & 2,1 & 2,6 & 3,0 & 3,9 \\
\hline Loratadin & 4,9 & 2,8 & 6,1 & 8,6 & 5 & 3,2 & 3,2 & 3,2 & Theophyllin & 2,1 & 2,8 & 3,4 & 11 \\
\hline$\ldots$ & & & & & & & & & Carbamazepin & 1,8 & 1,9 & 2,0 & 3,4 \\
\hline Roxithromycin & 3,7 & 11 & 15 & 23 & 5 & 13 & 13 & 13 & Diuron & 1,8 & 3,0 & 3,3 & 3,5 \\
\hline Clarithromycin & 4 & 8,7 & 15 & 25 & 10 & 8,7 & 9,4 & 10 & Sulfamethoxazol & 1,1 & 2,1 & 7,0 & 9,6 \\
\hline$\cdots$ & & & & & & & & & & & & & \\
\hline Metazachlor & 2,5 & 5,5 & 5,9 & 20 & 5 & 5,9 & 5,9 & 5,9 & & & & & \\
\hline$\cdots$ & & & & & & & & & & & & & \\
\hline Erythromycin & 1,8 & 5,5 & 6,4 & 390 & 0 & & & & & & & & \\
\hline
\end{tabular}

${ }^{\mathrm{a}} \mathrm{n}=135,{ }^{\mathrm{b}} \mathrm{n}=140,{ }^{\mathrm{c}} \mathrm{n}=18,{ }^{\mathrm{d}} \mathrm{n}=17,{ }^{\mathrm{e}} \mathrm{n}=231,{ }^{\mathrm{f}} \mathrm{n}=119,{ }^{\mathrm{g}}$ aus Reh et al. (2013) 
In der Waldecker Scholle treten die ATR-Konzentrationen mit nur geringen Schwankungen auf (Twiste-Quellen jeweils 17-22/ 33-35/ 27-31 ng/l; Itter-Quellen jeweils 5,1-7,9/ 2,36,0 ng/l). Außerdem sind sowohl die Konzentrationen als auch deren Schwankungsbreite im oberen Grundwasserstockwerk größer als im unteren Grundwasserleiter. Dies kann als Hinweis darauf interpretiert werden, dass ATR überwiegend als Langzeitkomponente im Speicher des Grundwasserleiters vorhanden ist. An der Gallusquelle ist die ATR-Konzentration auch gering und unterliegt leichten Schwankungen (Tab. 5.2). Diese konnten genutzt werden, um die Vermutung, dass ATR als Langzeitkomponente im Speicher des Grundwasserleiters vorhanden ist, zu bestätigen (Hillebrand et al. 2014). Das in der Gallusquelle gegenüber den Twiste-Quellen (Waldecker Scholle) um ungefähr Faktor zehn geringere Konzentrationsniveau spiegelt nicht nur die höhere Grundwasserneubildung und den geringeren Volumenanteil der Langzeitkomponente wider, sondern verweist auch auf eine größere Stärke der ATR-Eintragsquelle im Einzugsgebiet der Twiste.

Im Gegensatz zu ATR ist das ebenfalls zu den Triazinen gehörende Terbuthylazin (TERB) aktuell als Pflanzenschutzmittel in Deutschland zugelassen. Das Rückhaltevermögen wird aufgrund des Wasser-Oktanol-Verteilungskoeffizienten (log $\left.\mathrm{K}_{\mathrm{OW}} 3,1\right)$ etwas höher eingeschätzt als das von ATR (Hebig et al. 2014). In biologischen Abbauversuchen mit Grundwasser wurde ein biologischer Abbau mit geringen Raten beobachtet (Caracciolo et al. 2010). In der Waldecker Scholle tritt TERB mit einer Detektionshäufigkeit von 15\% oberhalb der BG im Grundwasser deutlich seltener auf als ATR (61\%). Das Vorkommen ist durch wiederholten Nachweis an einzelnen Messstellen mit Konzentrationen zwischen 3 und 93 ng/l ohne erkennbare saisonale Präferenz charakterisiert. TERB tritt in der Waldecker Scholle sowohl in Bachläufen, im oberen Grundwasserstockwerk sowie in den Quellen der Twiste und Itter auf. In der Gallusquelle hingegen wurde TERB im Laufe eines Jahres nie oberhalb der BG nachgewiesen.

Sowohl im Grundwasser der Waldecker Scholle als auch in der Gallusquelle findet sich Koffein. Koffein ist eine gut lösliche Substanz, die im Grundwasser einer schnellen Attenuation unterliegt (Hillebrand et al. 2012b). In der Gallusquelle ist es in 40\% der Proben hauptsächlich nach intensiven Niederschlagsereignissen im Quellwasser quantifizierbar, so dass Koffein bei hoher Beprobungsfrequenz von bis zu 8 Proben pro Tag als Indikator zur quantitativen Abschätzung des Einflusses unbehandelten Abwassers genutzt werden konnte (Hillebrand et al. 2012a). Die Koffein-Metaboliten Paraxanthin und Theobromin konnten ebenfalls regelmäßig (Nachweishäufigkeiten >BG 35 beziehungsweise 34\%) in der Gallusquelle nachgewiesen werden. Während eines Markierungsversuches mit Uranin und Koffein wurde ein 
im Verhältnis zum konservativ transportierten Uranin signifikanter Massenverlust des Koffeins beobachtet (Hillebrand et al. 2012b). Trotz der vermutlich durch biologische Vorgänge bedingten Verluste konnten die oben genannten Metabolite während dieses Markierungsversuches nur sporadisch nachgewiesen werden. Daher ist es sehr wahrscheinlich, dass Paraxanthin und Theobromin nicht im Grundwasserleiter gebildet, sondern über Abwasser (Schmidt \& Schoyerer 1966) in das System eingetragen werden. Im Gegensatz zur Gallusquelle kann Koffein in der Waldecker Scholle lediglich in einzelnen Beprobungsstellen des oberen Grundwasserstockwerks innerhalb der Quelleinzugsgebiete nachgewiesen werden. Metaboliten wurden hier nicht detektiert. In den Quellen der Waldecker Scholle war Koffein nicht quantifizierbar, obwohl aufgrund der identifizierten Eintragsfunktionen (Kanalsystem, Bachinfiltration) in diesem Untersuchungsgebiet von einem kontinuierlichen Eintrag dieses Alltagsprodukts auszugehen ist. Das im Gegensatz dazu sporadische Auftreten weist auf einen schnellen biologischen Abbau des Koffeins und auch der Metaboliten hin. Das bedeutet, dass nur in Systemen mit einer sehr schnellen Fließkomponente beziehungsweise nur bei kurzen Fließwegen im Quellaustritt bestimmbare Koffeinkonzentrationen erreicht werden.

Das detektierte Stoffspektrum erweitert sich in beiden Systemen nach intensiven Grundwasserneubildungsereignissen, wie beispielsweise großen Schneeschmelzen im Winter und Frühjahr. In der Waldecker Scholle traten bei solchen Ereignissen zusätzlich Antibiotika wie Clarithromycin, Erythromycin und Roxithromycin sowie das Antiallergikum Loratadin in einzelnen Messstellen jedoch nicht in den Quellen auf Abb. 5.2). In der Gallusquelle war auch nach solchen Ereignissen die Stoffanzahl geringer als in der Waldecker Scholle, dennoch wurden auch hier zusätzliche Stoffe festgestellt. Diese umfassten die Pestizide Isoproturon (Abb. 5.3) und Metazachlor (Hillebrand et al. 2014), Koffein (Hillebrand et al. 2012a), dessen Metabolite sowie teilweise die iodierten Röntgenkontrastmittel Iohexol (Abb. 5.3), Iomeprol und Iopromid und das Antibiotikum Sulfamethoxazol. Letzteres wurde auch in der Waldecker-Scholle gefunden, jedoch lediglich sporadisch und punktuell im Zusammenhang mit einer vermuteten Kanalleckage. 


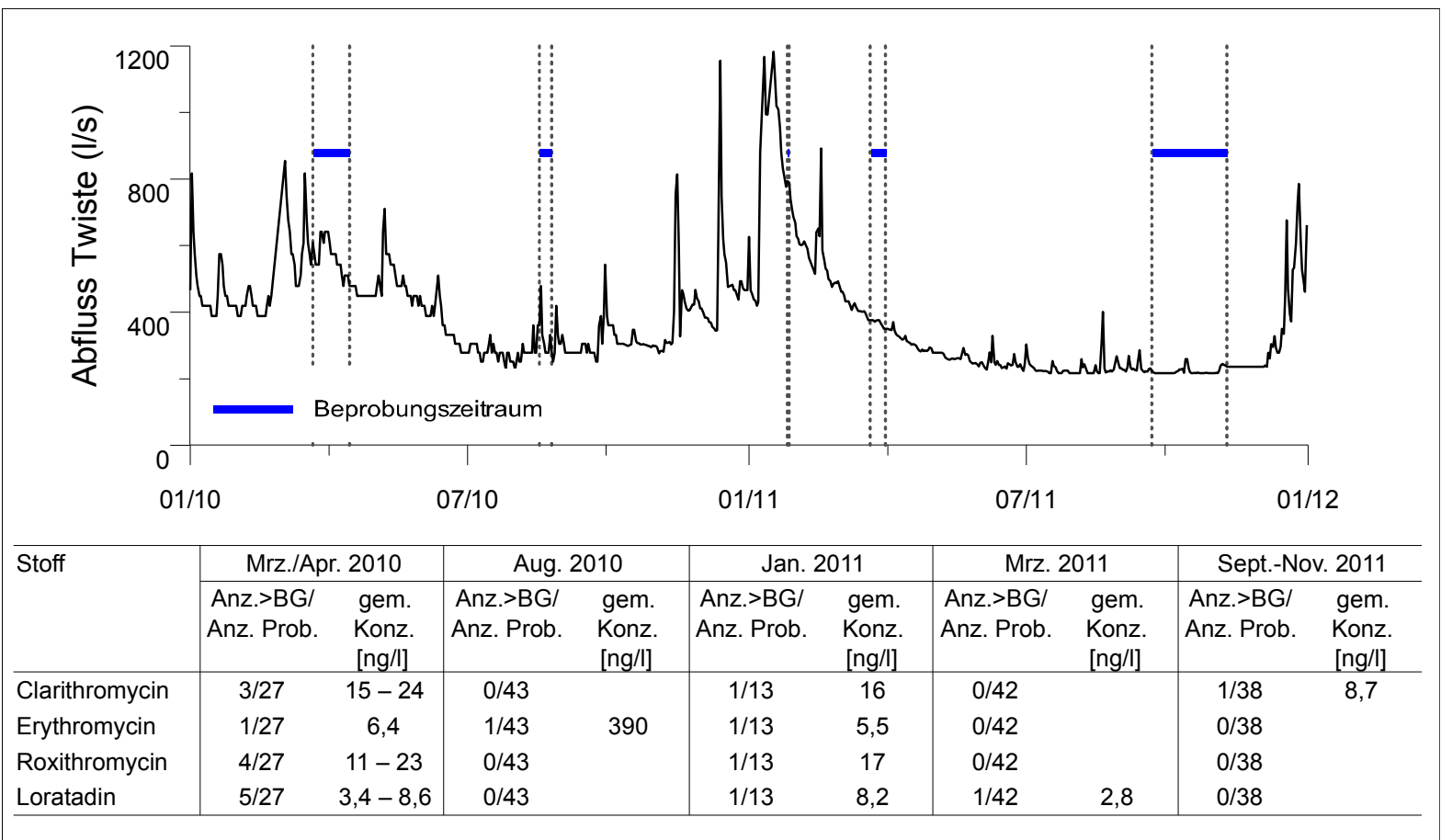

Abb. 5.2: Auftreten und Konzentrationen von Makrolid-Antibiotika und dem Antiallergikum Loratadin in Grundwasserproben der Waldecker Scholle mit Bezug zum Abfluss der Twiste

Das Anti-Epileptikum Carbamazepin gilt im Grundwasser als weitgehend persistent und hoch mobil (Clara et al. 2004). Es war in den Grundwasserproben der Waldecker Scholle in $13 \%$ der Proben in quantifizierbaren Konzentrationen vorhanden. Das Vorkommen ist an einzelne Messstellen gebunden, in denen die Konzentrationen in allen vier Beprobungen jeweils in der gleichen Größenordnung lagen, und wird als punktueller Eintrag über Kanalleckagen interpretiert. In den Quellabflüssen sind keine bestimmbaren Carbamazepinkonzentrationen ermittelt worden. In der Gallusquelle konnte Carbamazepin zwar in 52\% der 283 Proben nachgewiesen werden, jedoch lagen die Konzentrationen nur in 1,8\% der Proben oberhalb der BG. Aus der Auswertung der Zeitreihe (Abb. 5.3) wird ersichtlich, dass zu Zeiten mit hoher Quellschüttung und somit starker Verdünnung (hauptsächlich im Frühjahr zu Schneeschmelzereignissen) die Carbamazepinkonzentration unterhalb der Nachweisgrenze (NG) lag. Bei geringerer Schüttung und somit geringerer Verdünnung konnte Carbamazepin zwar nachgewiesen werden, die Konzentrationen waren jedoch nur nach kleineren Grundwasserneubildungsereignissen quantifizierbar. Eine mögliche Erklärung wäre, dass bei kleineren Ereignissen Carbamazepin freigesetzt oder eingetragen wird, ohne dass dabei die Konzentration unter die BG verdünnt wird. Somit sind die Quellen in beiden Untersuchungsgebieten durch das Fehlen beziehungsweise das Vorkommen minimaler Mengen mit lediglich sporadisch quantifizierbaren Konzentrationen von Carbamazepin charakterisiert. 
Obwohl die Anwendung dieses Antiepileptikums als dauerhaft und nicht saisonal begrenzt vorausgesetzt werden kann, sind die potentiellen Eintragsmengen gering (14 kg verschriebene Menge im Jahr 2010 in der Waldecker Scholle nach Reh et al. 2013), so dass Carbamazepin in den Quellabflüssen bis unter die BG verdünnt wird. Um in den Quellen quantifizierbare Konzentrationen $\mathrm{zu}$ erzeugen, sind besondere Ereignisse mit erhöhten Eintragsmengen notwendig.

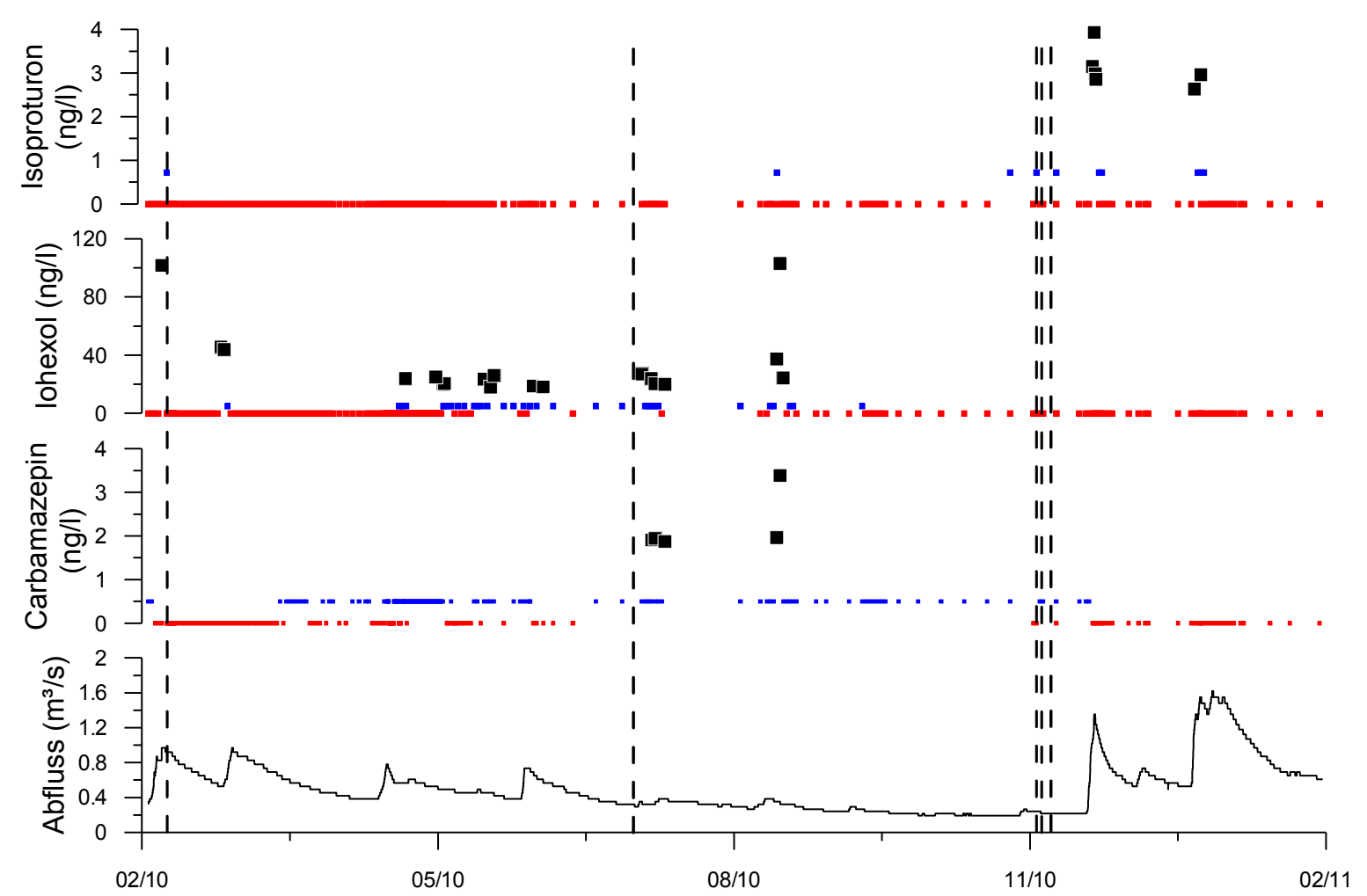

Abb. 5.3: Zeitliche Verteilung der Konzentrationen von Isoproturon, Iohexol und Carbamazepin an der

Gallusquelle. Die unterbrochenen senkrechten Linien stellen dokumentierte Überlaufereignisse des Regenrückhaltebeckens dar. (rote Symbole: Messwert < NG;

blaue Symbole: NG < Messwert < BG; schwarze Symbole: Messwert > BG)

Atenololsäure, ein Metabolit der Beta-Blocker Atenolol und Metoprolol (Barbieri et al. 2012, Kern et al. 2010), wurde ausschließlich in der Gallusquelle gefunden, hier immerhin mit einer Nachweishäufigkeit von $10 \%$ oberhalb der BG.

Von den analysierten Pestiziden werden, außer dem bereits oben erwähnten TERB, Isoproturon, Metazachlor, Diuron und Mecoprop aktuell eingesetzt. Isoproturon, ein schlecht in Wasser lösliches Phenylharnstoffderivat, gehört mit Absatzmengen >1.000 t (BVL 2011) gegenwärtig zu den am häufigsten eingesetzten Pflanzenschutzmitteln in Deutschland 
(s. Tab. 5.3). Daher ist in den landwirtschaftlichen Gebieten von einer flächenhaften Anwendung auszugehen. In der Waldecker Scholle wurde Isoproturon sowohl in Bachläufen als auch im oberen Grundwasserstockwerk mit Konzentrationen von 3-23 ng/l gefunden. Lokal in einem landwirtschaftlich genutzten Brunnen traten auch 32-78 ng/l auf. In den Quellen trat zwar kein Isoproturon auf, die Detektionshäufigkeit (Werte >BG) betrug jedoch in den untersuchten Grundwasserproben etwa 10\%. Auch in der Gallusquelle war Isoproturon quantifizierbar, jedoch sehr selten (2,1\%) und mit geringen Konzentrationen von 2,6-3,9 ng/l zeitgleich mit Metazachlor.

Tab. 5.3 Absatzmengen der untersuchten Pestizide

als Pflanzenschutzmittel in Deutschland

\begin{tabular}{ll}
\hline Stoffname & Absatzmenge $[\mathrm{t} / \mathrm{a}]^{a}$ \\
\hline Terbuthylazin & $250-1.000$ \\
Isoproturon & $>1.000$ \\
Metazachlor & $250-1.000$ \\
Diuron & $25-100^{b}$ \\
Mecoprop & $100-250$ \\
\hline
\end{tabular}

GWL: Grundwasserleiter

${ }^{a}$ Absatzmengen aus BVL (2011)

${ }^{\mathrm{b}}$ Absatzmenge 2007 danach nicht mehr erfasst

Diuron, ebenfalls ein Phenylharnstoffderivat, dessen Absatzmenge max. 1/10 der des Isoproturons entspricht, ist hingegen auch im Quellaustritt der Twiste (Waldecker Scholle) in allen untersuchten Proben vorhanden und tritt zusätzlich auch im unteren Grundwasserleiter auf. Die Detektionshäufigkeit oberhalb der BG betrug in den untersuchten Grundwasserproben 13\% mit Konzentrationen zwischen 3 und 20 ng/l. Der Einsatz von Diuron wurde 2003 durch die Pflanzenschutzanwendungsverordnung eingeschränkt, so dass zum Beispiel eine Anwendung auf Gleisanlagen, in Haus- und Kleingärten und auf Flächen mit Abläufen in die Kanalisation oder in Gewässer nicht mehr zulässig ist. Dass Diuron im Gegensatz zu anderen aktuell noch eingesetzten Pestiziden nicht in den Bachläufen mit influenten Verhältnissen vorkommt, deutet darauf hin, dass die Nachweise im Grundwasser, insbesondere in den Quellen der Twiste und im unteren Grundwasserleiter, auf zurückliegende Einträge zurückzuführen sind und damit auf eine Verweilzeit und Persistenz von mehreren Jahren hinweisen. Auch in anderen Studien wurde Diuron eine relativ hohe Persistenz in Boden und Grundwasser zugesprochen (Giacomazzi \& Cochet 2004). In der Gallusquelle war Diuron war nur sporadisch quantifizierbar (Detektionshäufigkeit >BG 1,8\%). Dieser Befund steht im Einklang mit der schnelleren Fließdynamik und der höheren Quellschüttung der 
Gallusquelle, die zum einen einen schnelleren Austrag aus dem System und zum anderen eine stärkere Verdünnung der eventuell verbliebenen Restkonzentrationen bewirken.

In der Gallusquelle wurde bis zu $72 \mathrm{ng} / \mathrm{l}$ Metazachlor detektiert. Im Vergleich zu den Konzentrationen der anderen nachgewiesenen Pestizide (s. Tab. 5.2) und angesichts der starken Verdünnung muss eine relativ starke Eintragsquelle vorliegen. Die Nachweishäufigkeit betrug 31\%, davon 17\% >BG. In der Waldecker Scholle trat Metazachlor nur in 3\% der Grundwasserproben zeitlich sporadisch, bevorzugt im Spätsommer und Herbst, mit Konzentrationen zwischen 6 und $20 \mathrm{ng} / 1 \mathrm{im}$ oberen Grundwasserstockwerk sowie zusätzlich in Bachwasserproben auf. Aufgrund geringer Halbwertszeiten (Allen \& Walker 1987, Beulke \& Malkomes 2001), ist anzunehmen, dass Metazachlor nur kurzzeitig im System persistiert und in Systemen, die durch eine langzeitige Fließkomponente dominiert werden, nur untergeordnet auftritt.

Mecoprop ist ein Pflanzenschutzmittel aus der Gruppe der Phenoxycarbonsäuren, das in der Waldecker Scholle aus einer Altablagerung freigesetzt wird (Reh et al. 2013). In der Gallusquelle und auch in den nicht von der Altablagerung beeinflussten Arealen der Waldecker Scholle war kein Mecoprop nachweisbar. Mecopropeinträge aus dem landwirtschaftlichen Bereich waren trotz einer in Deutschland gegenüber Diuron höheren jährlichen Absatzmenge als Pflanzenschutzmittel (100-250 t) in beiden Grundwassersystemen demnach nicht identifizierbar. Außerhalb der Landwirtschaft ist Mecoprop auch in Oberflächengewässern im Einflussbereich urbaner Gebiete nachgewiesen worden (Wittmer et al. 2010). Als eine mögliche Eintragsquelle wird der Einsatz als Biozid, zum Beispiel in Fassadenschutzanstrichen, angesehen. Auch dieser potentielle Eintragspfad führte in beiden Untersuchungsgebieten nicht zu bestimmbaren Grundwasserkonzentrationen und ist daher nicht erkennbar. Diese fehlenden Nachweise können als Hinweise auf eine im Vergleich zu Triazinen und Diuron geringere Persistenz von Mecoprop gesehen werden.

In beiden Systemen waren Triazole nachweisbar. Im nordhessischen Untersuchungsgebiet treten die unter anderem als Rostschutzmittel verwendeten Stoffe 1H-Benzotriazol (BT) und Tolyltriazol (TT) in großer flächiger Verbreitung auf. Beide Stoffe haben ein relativ geringes Sorptionsvermögen und sind mäßig biologisch abbaubar (Giger et al. 2006). Insbesondere im urban geprägten Einzugsgebiet der Twiste waren sie dauerhaft mit geringen Konzentrationsschwankungen quantifizierbar. Lokal hohe Konzentrationen bis hin zu einigen Mikrogramm pro Liter zeigen Einträge über Altablagerungen, das Kanalsystem und über Bachläufe mit influenten Verhältnissen an. In den Quellen der Twiste lagen die Konzentrationen zwischen 5 
und 20 ng/l BT sowie 5 und 46 ng/l TT mit einer Detektionshäufigkeit von 48\% (BT) beziehungsweise 52\% (TT). In der Gallusquelle wurden ebenfalls Triazole mit Konzentrationen in einer Größenordnung von 4-40 ng/l BT und 4-21 ng/l TT nachgewiesen, jedoch mit geringerer Detektionshäufigkeit (23\% BT, 13\% TT), die in Einklang mit der überwiegend landwirtschaftlichen Nutzung und der stärkeren Verdünnung steht.

Insgesamt acht der untersuchten Stoffe wurden weder in Grundwasserproben der Waldecker Scholle noch in der Gallusquelle in bestimmbaren Konzentrationen gefunden. Es handelt sich zum einen um die Medikamente Cetirizin, Diazepam, Tetrazepam, Naproxen, und Sertralin, deren Verschreibungsmengen während der Beprobungen gemäß Arzneiverordnungsreport (Schwabe \& Paffrath 2012) in Deutschland relativ gering waren (0,1-8 t jeweils in den Jahren 2010 und 2011), zum anderen aber auch um das relativ häufig verschriebene Arzneimittel Pantoprazol (45-59 t/a in 2010 und 2011) und den Metaboliten 4-OH-Diclofenac, dessen Ausgangssubstanz Diclofenac in Deutschland häufig verschrieben wird (44 beziehungsweise 42 t/a in 2010 und 2011) und zusätzlich rezeptfrei verkauft wird. Das Fehlen dieser eigentlich in größeren Mengen vorkommenden Substanzen im Vergleich mit ähnlich häufig verschriebenen Medikamenten, beispielsweise Carbamazepin (49 beziehungsweise 47 t/a in 2010 und 2011), weist auf einen natürlichen Rückhalt hin. Das iodierte Röntgenkontrastmittel Iopamidol wurde ebenfalls nicht gefunden, die anderen analysierten Röntgenkontrastmittel Iomeprol und Iohexol selten (Waldecker Scholle) oder nach besonderen Niederschlags- oder Grundwasserneubildungsereignissen (Gallusquelle). Da die Anwendung der Kontrastmittel auf Krankenhäuser und radiologische Facharztpraxen beschränkt ist, sind die Einsatzmengen in den kleinstädtisch bis ländlich geprägten Untersuchungsgebieten vermutlich gering.

\subsection{Schlussfolgerungen}

Triazine und Triazole sind systemunabhängig vorhanden und als Indikatoren des Stofftransports nutzbar. Atrazin ist auch in Karstsystemen über Jahrzehnte hinweg persistent. Der Triazin-Metabolit DIA war in beiden Systemen nicht in landwirtschaftlichen Gebieten quantifizierbar und ist in der Waldecker Scholle typisch für punktuelle Einträge im Stadtgebiet (Reh et al. 2013).

Mit Ausnahme der Triazine unterscheiden sich beide Untersuchungsgebiete hinsichtlich der am häufigsten gefundenen Stoffe. Während in der Waldecker Scholle ein hoher Anteil von Stoffen mit hoher Persistenz vorliegt (Triazole, Triazine, Carbamazepin, Diuron), kommen in 
der Gallusquelle auch kurzlebige Stoffe (Koffein mit Metaboliten, Metazachlor) vor. Da diese Stoffe häufig nur für kurze Zeit auftreten, wird eine zeitlich hoch aufgelöste Probenahmefrequenz benötigt, um sicherzustellen, dass der jeweilige Stoffdurchgang erfasst wird. Auch wenn die Studie auf die Untersuchung von zwei Gebieten begrenzt ist, lässt sich erkennen, dass die Charakteristika der gefundenen Stoffe die jeweilige Fließdynamik widerspiegeln. Schnell abbaubare Verbindungen sind nur in schnellen Systemen in den Quellen detektierbar.

Die mit der durchgeführten Analytik zur Verfügung stehende große Anzahl detektierbarer Substanzen bietet die Möglichkeit diesen Unterschieden gerecht zu werden und Stoffe als Indikatoren für Transportprozessse auszuwählen, die die spezifischen Charakteristika der verschiedenen Einzugsgebiete und Grundwasserleiter reflektieren.

Im urban geprägten System tritt ein deutlich größeres Stoffspektrum auf als in Systemen mit vorwiegend landwirtschaftlicher Nutzung. Das Stoffspektrum zeigt somit an, ob sich Stadt-/ Siedlungsgebiete im Einzugsgebiet befinden.

In den Quellaustritten beider Systeme sind nur Stoffe mit großflächigem Eintrag, wie Pestizide, oder großen Einsatzmengen, beispielsweise Koffein, konstant vertreten. Das Konzentrationsniveau ist so gering, dass Grenzwerte der Trinkwasserverordnung oder gesundheitliche Orientierungswerte (UBA 2003) für nicht in der Trinkwasserverordnung aufgeführte Stoffe hier nicht überschritten werden. Aus Punktquellen freigesetzte Stoffe sind nur lokal begrenzt detektierbar. In Einzugsgebieten mit hoher Schüttung führen natürliche Rückhalteprozesse und Verdünnung zur Konzentrationsminderung. Eine flächendeckende Untersuchung ist daher zur Erfassung eines breiteren Stoffspektrums besser geeignet und notwendig, wenn Punktquellen identifiziert werden sollen.

In landwirtschaftlichen Gebieten treten erwartungsgemäß nur wenige der analysierten Verbindungen auf. Insbesondere humanpharmazeutische Produkte fehlen hier weitgehend oder sind an bestimmte hydrodynamische Situationen gebunden. Als aktuell eingesetzte Pflanzenschutzmittel werden Metazachlor, Isoproturon und Terbuthylazin in den beiden untersuchten Karstgebieten zwar gefunden, die Detektionshäufigkeit ist aber weitaus geringer als die des Atrazins und des Triazin-Metaboliten DEA. 


\subsection{Danksagung}

Die Untersuchungen zur Waldecker Scholle wurden gefördert vom Hessischen Landesamt für Umwelt und Geologie HLUG, Wiesbaden sowie von der Energie Waldeck-Frankenberg EWF GmbH. Wir danken hierfür insbesondere Frau Schlösser-Kluger und Frau Jaeger-Wunderer (HLUG) sowie Herrn Schaller und Herrn Wilke (EWF). Wir danken den Mitarbeitern des Wasswerkes Hermentingen, besonders Herrn Peter Knaus, für die Hilfe bei den Untersuchungen an der Gallusquelle. Weiterhin danken wir dem Bundesministerium für Bildung und Forschung (BMBF) für die Unterstützung im Rahmen der Fördermaßnahme Nachhaltiges Wassermanagement (NaWaM-RiSKWa; Förderkennzeichen 02WRS1277A, AGRO, "Risikomanagement von Spurenstoffen und Krankheitserregern in ländlichen Karsteinzugsgebieten").

\subsection{Literatur}

Allen, R., Walker, A.: The influence of soil properties on the rates of degradation of metamitron, metazachlor and metribuzin. Pestic. Sci. 18, 95-111 (1987)

Atkinson, T.C.: Diffuse flow and conduit flow in limestone terrain in the Mendip Hills, Somerset (Great Britain). J. Hydrol. 35, 93-110 (1977)

Baran, N., Lepiller, M., Mouvet, C.: Agricultural diffuse pollution in a chalk aquifer (Trois Fontaines, France): Influence of pesticide properties and hydrodynamic constraints. J. Hydrol. 358, 56-69 (2008)

Barbieri, M., Licha, T., Nödler, K., Carrera, J., Ayora, C., Sanchez-Vila, X.: Fate of $\beta$-blockers in aquifer material under nitrate reducing conditions: batch experiments. Chemosphere 89, 1272-1277 (2012)

Beulke, S., Malkomes, H.-P.: Effects of the herbicides metazachlor and dinoterb on the soil microflora and the degradation and sorption of metazachlor under different environmental conditions. Biol. Fertil. Soils 33, 467-471 (2001)

BVL: Absatz an Pflanzenschutzmitteln in der Bundesrepublik Deutschland Ergebnisse der Meldungen gemäß § 19 Pflanzenschutzgesetz für das Jahr 2010. 
http://www.bvl.bund.de/SharedDocs/Downloads/04_Pflanzenschutzmittel/

meld_par_19_2010.pdf (2011). Zugegriffen: 01. Juli 2013

Caracciolo, A.B., Fajardo, C., Grenni, P., Sacca, M.L., Amalfitano, S., Ciccoli, R., Martin, M., Gibello, A.: The role of a groundwater bacterial community in the degradation of the herbicide terbuthylazine. FEMS Microbiol. Ecol. 71, 127-136 (2010)

Clara, M., Strenn, B., Kreuzinger, N.: Carbamazepine as a possible anthropogenic marker in the aquatic environment: investigations on the behaviour of carbamazepine in wastewater treatment and during groundwater infiltration. Water Res. 38, 947-954 (2004)

DWD: http://www.dwd.de/bvbw/generator/DWDWWW/Content/Oeffentlichkeit/KU/ KU2/KU21/klimadaten/german/nieder_6190_akt_html,templateId=raw, property=publi cationFile.html/nieder_6190_akt_html.html. Zugegriffen: 01. Juni. 2013

Einsiedl, F.: Flow system dynamics and water storage of a fissured-porous karst aquifer characterized by artificial and environmental tracers. J. Hydrol. 312, 312-321 (2005)

Estévez, E., del Carmen Cabrera, M., Molina-Díaz, A., Robles-Molina, J., del Pino Palacios-Díaz, M.: Screening of emerging contaminants and priority substances (2008/105/EC) in reclaimed water for irrigation and groundwater in a volcanic aquifer (Gran Canaria, Canary Islands, Spain). Sci. Total Environ. 433, 538-546 (2012)

Ford, D.C., Williams, P.: Karst hydrogeology and Geomorphology. 562 S.; John Wiley \& Sons Ltd., West Sussex (2007)

Geyer, T., Birk, S., Licha, T., Liedl, R., Sauter, M.: Multitracer test approach to characterize reactive transport in karst aquifers. Ground Water 45, 36-45 (2007)

Geyer, T., Birk, S., Reimann, T., Dörfliger, N., Sauter, M.: Differentiated characterization of karst aquifers: some contributions. Carbonates Evaporites 28, 41-46 (2013)

Geyer, T., Selg, M., Gudera, T., Sauter, M.: Langzeitabflussverhalten der Gallusquelle und des Blautopfs - relative Bedeutung der Matrix und des Karströhrensystems. Laichinger Höhlenfreund 46, 63-74 (2011)

Giacomazzi, S., Cochet, N.: Review - Environmental impact of diuron transformation: a review. Chemosphere 56, 1021-1032 (2004) 
Giger, W., Schaffner, C., Kohler, H.-P.E.: Benzotriazole and tolyltriazole as aquatic contaminants. 1. Input and occurrence in rivers and lakes. Environ. Sci. Technol. 40, 7186-7192 (2006)

Heberer, T., Mechlinski, A., Fanck, B., Knappe, A., Massmann, G., Pekdeger, A., Fritz, B.: Field studies on the fate and transport of pharmaceutical residues in bank filtration. Ground Water Monit. R. 24, 70-77 (2004)

Hebig, K.H., Nödler, K., Licha, T. Scheytt, T.J.: Impact of materials used in lab and field experiments on the recovery of organic micropollutants. Sci. Total Environ. 473-474, 125-131 (2014)

Heinz, B., Birk, S., Liedl, R., Geyer, T., Straub, K. L., Andresen, J., Bester, K., Kappler, A.: Water quality deterioration at a karst spring (Gallusquelle, Germany) due to combined sewer overflow: evidence of bacterial and micro-pollutant contamination. Environ.

Geol. 57, 797-808 (2009)

Hillebrand, O., Nödler, K., Geyer, T., Licha, T.: Investigating the dynamics of two herbicides at a karst spring in Germany - consequences for sustainable raw water management. Sci. Total Environ. (accepted manuscript) DOI 10.1016/j.scitotenv.2014.02.117 (2014)

Hillebrand, O., Nödler, K., Licha, T., Sauter, M., Geyer, T.: Caffeine as an indicator for the quantification of untreated wastewater in karst systems. Water Res. 46, 395-402 (2012a)

Hillebrand, O., Nödler, K., Licha, T., Sauter, M., Geyer, T.: Identification of the attenuation potential of a karst aquifer by an artificial dualtracer experiment with caffeine. Water Res. 46, 5381-5388 (2012b)

HLUG: Geologische Übersichtskarte von Hessen, Auflage 5; Wiesbaden (2008)

Jablonowski, N.D., Schäffer, A., Burauel, P.: Still present after all these years: persistence plus potential toxicity raise questions about the use of atrazine. Environ. Sci. Pollut. R. 18, 328$331(2011)$

Käding, K.-C.: Der Zechstein in der Stratigraphischen Tabelle von Deutschland 2002. Newsl. Stratigr. 41, 123-127 (2005) 
Kern, S., Baumgartner, R., Helbling, D.E., Hollender, J., Singer, H., Loos, M.J., Schwarzenbach, R.P., Fenner, K.: A tiered procedure for assessing the formation of biotransformation products of pharmaceuticals and biocides during activated sludge treatment. J. Environ. Monitor. 12, 2100-2111 (2010)

Kolpin, D.W., Furlong E.T., Meyer M.T., Thurman E.M., Zaugg S.D., Barber L.B., Buxton H.T.: Pharmaceuticals, hormones, and other organic wastewater contaminants in US streams, 1999-2000: a national reconnaissance. Environ. Sci. Technol. 36, 1202-1211 (2002)

Kulick, J.: Erläuterungen zur Geologischen Karte von Hessen 1:25.000, Blatt 4719 Korbach. 2. Aufl., 272 S.; Wiesbaden (1997)

Massmann, G., Dunnbier, U., Heberer, T., Taute, T.: Behaviour and redox sensitivity of pharmaceutical residues during bank filtration -Investigation of residues of phenazone -type analgesics. Chemosphere 71, 1476-1485 (2008)

Metcalfe, C.D., Beddows, P.A., Bouchot, G.G., Metcalfe, T.L., Li, H., Van Lavieren, H.: Contaminants in the coastal karst aquifer system along the Caribbean coast of the Yucatan Peninsula, Mexico. Environ. Pollut. 159, 991-997 (2011)

Morasch, B.: Occurrence and dynamics of micropollutants in a karst aquifer. Environ. Pollut. 173, 133-137 (2013)

Nödler, K., Licha, T., Bester, K., Sauter, M.: Development of a multi-residue analytical method, based on liquid chromatography-tandem mass spectrometry, for the simultaneous determination of 46 micro-contaminants in aqueous samples. J. Chromatogr. A 1217, 6511-6521 (2010)

PflSchAnwV: Verordnung über Anwendungsverbote für Pflanzenschutzmittel (Pflanzenschutz-Anwendungsverordnung). Pflanzenschutz-Anwendungsverordnung vom 10. November 1992 (BGBl. I S. 1887)

Reh R., Licha T., Geyer T., Nödler, K., Sauter M.: Occurrence and spatial distribution of organic micro-pollutants in a complex hydrogeological karst system during low flow and high flow periods, results of a two-year study. Sci. Total Environ. 443, 438-445 (2013)

Rowden, R.D., Liu, H., Libra, R.D.: Results from the Big Spring basin water quality monitoring and demonstration projects, Iowa, USA. Hydrogeol. J. 9, 487-497 (2001) 
Sacher, F., Ehmann, M., Gabriel, S., Graf, C., Brauch, H.J.: Pharmaceutical residues in the river Rhine - results of a one-decade monitoring programme. J. Environ. Monit. 10, 664$670(2008)$

Sauter, M.: Quantification and forecasting of regional groundwater flow and transport in a karst aquifer (Gallusquelle, Malm, SW. Germany). 150 S.; Tübinger Geowissenschaftliche Arbeiten C13, Tübingen (1992)

Schmidt, G., Schoyerer, R.: Zum Nachweis von Coffein und seinen Metaboliten im Harn. International Journal of Legal Medicine 57, 402-409 (1966)

Schraft, A., Fritsche, J.G., Hemfler, M., Mittelbach, G., Rambow, D., Tangermann, T.: Die hydrogeologischen Einheiten Nordhessens, ihre Grundwasserneubildung und ihr nutzbares Grundwasserdargebot (Ldkrs. Waldeck-Frankenberg, Kassel, Schwalm-Eder, WerraMeißner, Hersfeld-Rotenburg, Fulda und Stadt Kassel). Geol. Jb. Hessen 129, 27-53 (2002)

Schwabe, U., Paffrath, D. (Hrsg.): Arzneiverordnungs-Report 2012: Aktuelle Daten, Kosten, Trends und Kommentare. 1.142 S., Springer-Verlag (2012)

Schwarzenbach, R.P., Escher, B.I., Fenner, K., Hofstetter, T.B., Johnson, C.A., von Gunten, U., Wehrli, B.: The challenge of micropollutants in aquatic systems. Science 313, 1072-1077 (2006)

Ternes T.: The occurrence of micopollutants (sic!) in the aquatic environment: a new challenge for water management. Water Sci. Technol. 55(12), 327-332 (2007)

UBA: Bewertung der Anwesenheit teil- oder nicht bewertbarer Stoffe im Trinkwasser aus gesundheitlicher Sicht. Empfehlung des Umweltbundesamtes nach Anhörung der Trinkwasserkommission, Bundesgesundheitsbl-Gesundheitsforsch-Gesundheitsschutz 46, 249251 (2003)

White, W.B.: Conceptual models for carbonate aquifers. Ground Water 7, 15-21 (1969)

WHO: Guidelines for drinking-water quality, 4th edition. Elektronisch abrufbar: http://whqlibdoc.who.int/publications/2011/9789241548151_eng.pdf (2011). Zugegriffen: 21.11.2013. 
Wittmer I.K., Bader, H.-P., Scheidegger, R. Singer, H., Lück, A. Hanke, I. Carlsson, C. Stamm, C.: Significance of urban and agricultural land use for biocide and pesticide dynamics in surface waters. Water Res. 44, 2850-2862 (2010) 


\section{Anhang}

Tab. 5.4: Untersuchte Stoffe, Anwendungsbereiche und Bestimmungsgrenzen nach Nödler et al. (2010) und Reh et al. (2013)

\begin{tabular}{|c|c|c|c|}
\hline \multirow[t]{2}{*}{ Anwendung } & \multirow[t]{2}{*}{ Stoffname } & \multicolumn{2}{|c|}{ Bestimmungsgrenzen (ng/l) } \\
\hline & & Waldecker Scholle & Gallusquelle \\
\hline \multirow[t]{6}{*}{$\begin{array}{l}\text { Pflanzenschutzmittel und Biozide } \\
\text {. }\end{array}$} & Diuron & 3,3 & 2,6 \\
\hline & Isoproturon & 3 & 2,4 \\
\hline & Metazachlor & 1,8 & 1,4 \\
\hline & Atrazin & 1,4 & 1,1 \\
\hline & Mecoprop & 1,2 & 1 \\
\hline & Terbuthylazin & 3 & 2,4 \\
\hline \multirow[t]{2}{*}{ Metabolite von Pflanzenschutz-mitteln und Bioziden } & Desethylatrazin & 1,7 & 1,4 \\
\hline & Desisopropylatrazin & 5,6 & 4,5 \\
\hline \multicolumn{4}{|l|}{ Arzneimittel } \\
\hline \multirow[t]{5}{*}{ Schmerzmittel } & Phenazon & 2 & 1,6 \\
\hline & Paracetamol & 3,7 & 3 \\
\hline & Diclofenac & 2 & 1,6 \\
\hline & Ibuprofen & 3,6 & 2,9 \\
\hline & Naproxen & 4,8 & 3,8 \\
\hline \multirow[t]{2}{*}{ Antiallergika } & Cetirizin & 2,2 & 1,8 \\
\hline & Loratadin & 2,7 & 2,2 \\
\hline \multirow[t]{5}{*}{ Antibiotika } & Sulfamethoxazol & 2,6 & 2,1 \\
\hline & Roxithromycin & 9,5 & 7,6 \\
\hline & Clarithromycin & 7,5 & 6 \\
\hline & Erythromycin & 4,3 & 3,4 \\
\hline & Trimethoprim & 2,5 & 2 \\
\hline \multirow[t]{4}{*}{ Antiepileptika, Sedativa } & Carbamazepin & 2,2 & 1,8 \\
\hline & Diazepam & 1,4 & 1,1 \\
\hline & Primidon & 2,7 & 2,2 \\
\hline & Tetrazepam & 2,5 & 2 \\
\hline \multirow[t]{4}{*}{ Antidrepressiva } & Haloperidol & 4 & 3,2 \\
\hline & Citalopram & 3,2 & 2,6 \\
\hline & Fluoxetin & 16 & 12,8 \\
\hline & Sertralin & 16 & 12,8 \\
\hline \multirow[t]{3}{*}{ Blutdrucksenker } & Metoprolol & 4,1 & 3,3 \\
\hline & Sotalol & 4,8 & 3,8 \\
\hline & Atenolol & 3,5 & 2,8 \\
\hline Anti-Östrogen & Tamoxifen & 6 & 3,2 \\
\hline \multirow[t]{4}{*}{ Iodierte Röntgenkontrastmittel } & Iohexol & 21 & 16,8 \\
\hline & Iomeprol & 18 & 14,4 \\
\hline & Iopamidol & 19 & 15,2 \\
\hline & Iopromid & 18 & 14,4 \\
\hline \multirow[t]{3}{*}{ Lipidsenker } & Clofibrinsäure & 3,4 & 2,7 \\
\hline & Bezafibrat & 3,5 & 2,8 \\
\hline & Gemfibrocil & 2 & 1,6 \\
\hline Protonenpumpenhemmer & Pantoprazol & 4,8 & 3,8 \\
\hline \multirow[t]{4}{*}{ Metabolite von Arzneimitteln } & Desamino-SMX & 1,5 & 2,4 \\
\hline & 4-Nitro-SMX & 1 & 2,4 \\
\hline & 4-OH-Diclofenac & 3 & 2,4 \\
\hline & Atenololsäure & 4 & 3,2 \\
\hline \multirow[t]{2}{*}{ Korrosionsschutz } & 1H-Benzotriazol & 4,7 & 3,8 \\
\hline & Tolyltriazol & 4,9 & 3,9 \\
\hline Alltagsprodukte, Stimmulanzien & Koffein & 4,3 & 3,4 \\
\hline
\end{tabular}


5 Charakterisierung zweier Karstsysteme mit Hilfe organischer Spurenstoffe

\begin{tabular}{lllc}
\hline Anwendung & Stoffname & \multicolumn{2}{l}{ Bestimmungsgrenzen (ng/l) } \\
\cline { 3 - 4 } & & Waldecker Scholle & Gallusquelle \\
\hline Metabolite von Alltagsprodukten und Stimmulanzien & Theobromin & 5,1 & 4,1 \\
& Paraxanthin & 3,2 & 2,6 \\
& 1-Methylxanthin & 21 & 16,8 \\
& Theophyllin & 3,4 & 2,7 \\
& 3-Methylxanthin & 28 & 22,4 \\
& Benzoylecgonin & 2,3 & 1,8 \\
& Cotinin & 4 & 3,2 \\
\hline
\end{tabular}





\section{Schlussfolgerungen}

\subsection{Möglichkeiten der Nutzung organischer Spurenstoffe zur Charakterisierung komplexer Grundwassersysteme}

Die Analysen der Untersuchungsgebiete "Waldecker Scholle“ und des Einzugsgebiets der Gallusquelle zeigen, dass organische Spurenstoffe auch in komplexen Grundwassersystemen wie Karstgrundwasserleitern vorkommen, wodurch die Grundvoraussetzung für deren Nutzung als Indikatoren erfüllt wird. In beiden Gebieten reflektieren die auftretenden Stoffe und deren Verteilungsmuster die hydrogeologischen Verhältnisse. Rückschlüsse auf die Systemeigenschaften lassen dabei sowohl räumliche Verteilungsmuster an mehreren Messstellen als auch zeitliche Verteilungsmuster an einer Quelle zu.

Räumliche Verteilungsmuster können zur Lokalisierung von Quelleinzugsgebietsgrenzen genutzt werden. Außerdem liefern sie Hinweise zur Identifikation von punktuellen Eintragsquellen und deren Auswirkung auf den Grundwasserleiter sowie dass Attenuationpotential der daraus freigesetzten Stoffe. Dabei lässt die stoffliche Zusammensetzung eine Differenzierung unterschiedlicher Eintragsmodi zu, beispielsweise Einträge aus Altablagerungen gegenüber Einträgen aus dem Kanalleckagen oder flächenhaften Einträgen landwirtschaftlicher Nutzflächen. Weiterhin kann anhand der räumlichen Stoffverteilung die hydraulische Wirksamkeit von geringleitenden Zwischenschichten und Störungszonen abgeschätzt werden. Da die Stoffe zum Zeitpunkt der Untersuchung bereits im Grundwasser vorhanden sind, können durch die Untersuchung persistenter Stoffe auch einige Jahrzehnte andauernde Fließprozesse erkundet werden. Auch Annahmen hinsichtlich der Vulnerabilität lassen sich validieren. Damit ist die Interpretation der räumlichen Verteilung organischer Spurenstoffe zur Charakterisierung sowohl kleinräumiger als auch großräumiger Zusammenhänge geeignet.

Bei Untersuchungen an einer Karstquelle reflektiert das auftretende Stoffspektrum auch die Altersstruktur des Wassers: Stoffe mit hoher Persistenz, deren Haupteintragsperiode Dekaden zurückliegt, verweisen auf eine Komponente mit entsprechender Verweilzeit, wie das Beispiel Atrazin zeigt. Stoffe mit einem hohen Attenuationspotential, wie beispielsweise Koffein, deuten auf eine schnelle Fließkomponente hin. Das bedeutet, dass die Art der in der Quelle auftretenden Stoffe auch ohne aufwändige zeitlich hochaufgelöste Beprobung Rück- 
schlüsse auf Verweilzeiten im System zulässt. Organische Spurenstoffe spiegeln somit auch die unterschiedlichen Porositäten eines Karstsystems wider.

In den untersuchten Karstquellen wurden nur Stoffe mit hoher Persistenz oder großen Eintragsmengen nachgewiesen. Generell muss also mit einer hohen Verdünnung als auch mit einem natürlichen Rückhalt der auftretenden Stoffkonzentrationen gerechnet werden. Punktuell eingetragene Stoffe, beispielsweise aus Altablagerungen, wurden in den Quellen beider Untersuchungsgebiete nicht in quantifizierbaren Konzentrationen nachgewiesen. Damit liefert die Untersuchung organischer Spurenstoffe auch mittels einer Quellbeprobung Hinweise auf die Eintragsarten und Mengen.

Ebenso wie für andere hydrochemische Verfahren gilt, dass für eine sichere Interpretation weitere Erkenntnisse über die geologischen Rahmenbedingungen vorliegen müssen. Die Nutzung von organischen Spurenstoffen als Indikatoren ist von Stoffeinträgen abhängig. Diese können auf Grundlage der Landnutzung abgeschätzt werden. Ob tatsächlich ein nutzbares Spektrum organischer Spurenstoffe vorhanden ist, zeigt sich jedoch erst nach einer Erstuntersuchung.

Die Nutzung organischer Spurenstoffe ist nicht auf Karstsysteme beschränkt. Sie kann überall dort angewendet werden, wo eine anthropogene Beeinflussung des Grundwassers vorhanden ist.

\subsection{Organische Spurenstoffe zur Charakterisierung von Grundwasserleitern im Vergleich mit etablierten hydrochemischen Methoden}

Etablierte hydrochemische Untersuchungsmethoden umfassen in der Regel nur Datensätze mit wenigen Analysenparametern. Daher werden häufig Kombinationen verschiedener Untersuchungsmethoden eingesetzt. Durch die modernen Methoden der Analytik organischer Stoffe entsteht die Möglichkeit der effizienten Erfassung eines großen Stoffspektrums mit nur einem Analysengang. Damit erhöhen sich die Chancen, die Stoffe zu identifizieren, die die Eigenschaften des jeweiligen Systems reflektieren.

Die in Kap. 2 beschriebenen und zitierten Methoden lassen sich gemäß Tab. 6.1 einteilen. In diese Systematik reiht sich die Nutzung organischer Spurenstoffe reiht sich in die Verfahren anthropogen freigesetzter Stoffe ein, die nicht mit Absicht einer hydrogeologischen Erkundung in das Grundwasser freigesetzt werden (passive Freisetzung). Sie sind rein anthropo- 
gener Herkunft, was auch für die Mehrzahl der häufig genutzten Umwelttracer gilt, jedoch teilweise mit der Einschränkung geringer natürlicher Hintergrundkonzentrationen (beispielsweise von ${ }^{3} \mathrm{H}$ und $\mathrm{SF}_{6}$ ). Unter den organischen Spurenstoffen finden sich in der aquatischen Umwelt neben reaktiven Stoffen auch weitgehend persistente Stoffe. Hierin unterscheiden sie sich von den bekannten Umwelttracern, deren Konzentrationen beziehungsweise Verhältnisse sich durch Zerfall der radioaktive Isotope oder durch Verflüchtigung (FCKW) verändern.

Durch ihre rein anthropogene Herkunft existiert für organische Spurenstoffe keine geogene Hintergrundkonzentration. Hierin besteht ein entscheidender Vorteil gegenüber allen Methoden, die natürlich vorkommende Stoffe oder Stoffe nutzen, die wie einige Umwelttracer, auch in geringen Konzentrationen natürlich vorkommen. Die fehlende geogene Hintergrundkonzentration erlaubt eine Nutzung zur Charakterisierung von Grundwassersystemen auch auf einem sehr geringen Konzentrationsniveau, das ausschließlich durch die Empfindlichkeit der Analysenmethode limitiert ist. Dieser Vorteil besteht auch gegenüber einigen künstlich eingegebenen Markierungsstoffen, die als Salze oder über die Intensität der Fluoreszenz gemessen werden und sich somit auch von einer natürlichen Hintergrundkonzentration oder -intensität abheben müssen. 
Tab. 6.1: Systematik etablierter hydrochemischer Methoden zur hydrogeologischen Charakterisierung mit Beispielstoffen, Eintragsarten und Zielsetzungen abgeleitet nach Åberg (1994), Barbieri et al. (2005), Cook \& Solomon (1997), Field \& Pinsky (2000), Furtak \& Langguth (1967), Gat (2010), Geyer et al. (2007), Goldscheider et al. (2008), Hölting \& Matthess (1963), Johannesson (1997), Käss et al. (2004), Katz et al. (2004), Kendall \& McDonnell (1998), Long et al. (2008), Maloszewski \& Zuber (1982), Pinault et al. (2001), Piper (1944), Sidle (1998), Solomon et al. (1998), Sültenfuss \& Massmann (2004), Tallini et al. (2014)

\begin{tabular}{|c|c|c|c|c|c|}
\hline \multirow{2}{*}{\multicolumn{2}{|c|}{$\begin{array}{l}\text { künstliche Eingabe } \\
\text { künstliche Markierungsstoffe }\end{array}$}} & \multicolumn{4}{|c|}{ keine Eingabe } \\
\hline & & \multirow{2}{*}{\multicolumn{2}{|c|}{ natürliche Herkunft }} & \multicolumn{2}{|c|}{ anthropogene Herkunft } \\
\hline \multicolumn{2}{|l|}{ Eintragsart } & & & & \\
\hline \multirow{2}{*}{\multicolumn{2}{|c|}{ punktuell }} & \multirow{2}{*}{\multicolumn{2}{|c|}{ flächig }} & \multirow{2}{*}{\multicolumn{2}{|c|}{ flächig und punktuell }} \\
\hline & & & & & \\
\hline \multicolumn{2}{|c|}{$\begin{array}{l}\text { Verhalten im Grundwasser (überwiegend) } \\
\text { konservativ nicht konservativ } \\
\text { Beispielstoffe }\end{array}$} & stabil & nicht konservativ & konservativ & nicht konservativ \\
\hline \multirow{5}{*}{$\begin{array}{l}\text { Uranin } \\
\text { Eosin } \\
\text { Salze: } \\
\text { Chlorid } \\
\text { Bromid }\end{array}$} & Sulforhodamin G & \multirow{5}{*}{$\begin{array}{l}\text { Hauptionen } \\
\text { Isotope des } \\
\text { Wassers: } \\
{ }^{2} \mathrm{H} \\
{ }^{18} \mathrm{O}\end{array}$} & Hauptionen & \multirow[t]{3}{*}{ Hauptionen } & Hauptionen \\
\hline & Tinopal & & REY & & REY \\
\hline & $\begin{array}{l}\text { Koffein } \\
\text { Gase }\end{array}$ & & \multirow{3}{*}{$\begin{array}{c}\text { Gel. nat. Isot.: } \\
{ }^{87} \mathrm{Sr} /{ }^{86} \mathrm{Sr} \\
{ }^{13} \mathrm{C} /{ }^{12} \mathrm{C} \\
{ }^{14} \mathrm{C} \\
{ }^{13} \mathrm{C}-\mathrm{DIC} \\
{ }^{15} \mathrm{~N} /{ }^{14} \mathrm{~N} \\
\left({ }^{3} \mathrm{H}\right) \\
\left(\mathrm{SF}_{6}\right)\end{array}$} & & $\begin{array}{c}\text { Umwelttracer: } \\
{ }^{3} \mathrm{H} \\
{ }^{3} \mathrm{H} / \mathrm{He}\end{array}$ \\
\hline & $\begin{array}{c}\text { Partikel } \\
\text { (Bärlappsporen) }\end{array}$ & & & $\begin{array}{l}\text { org. } \\
\text { Spurenstoffe }\end{array}$ & $\begin{array}{l}{ }^{85} \mathrm{Kr} / \mathrm{Kr} \\
{ }^{36} \mathrm{Cl} \\
\mathrm{FCKW}^{\mathrm{FCKW}} \\
\mathrm{SF}_{6}\end{array}$ \\
\hline & & & & & $\begin{array}{l}\text { org. } \\
\text { Spurenstoffe }\end{array}$ \\
\hline \multicolumn{2}{|c|}{ Ermittelbare Eigenschaften } & & \multicolumn{3}{|c|}{ ( ) = geringe natürliche Hintergrundkonz./-Aktivität } \\
\hline \multirow{7}{*}{$\begin{array}{l}\text { hydraulische } \\
\text { Verbindungen } \\
\text { Fließrichtung } \\
\text { Geschwindigkeit } \\
\text { Transport- } \\
\text { parameter } \\
\text { Verweilzeit }\end{array}$} & \multirow{7}{*}{$\begin{array}{c}\text { hydraulische } \\
\text { Verbindungen } \\
\text { Fließrichtung } \\
\text { Attenuations- } \\
\text { potential }\end{array}$} & \multirow{7}{*}{$\begin{array}{l}\text { Verweilzeit } \\
\text { Mischungs- } \\
\text { verhältnisse } \\
\text { Höhenlage } \\
\text { Einzugsgebiet } \\
\text { Zonen GW- } \\
\text { Neubildung }\end{array}$} & \multirow{7}{*}{$\begin{array}{l}\text { Verweilzeit } \\
\text { Mischungs- } \\
\text { verhältnisse } \\
\text { Chem. und biol. } \\
\text { Prozesse } \\
\text { Zonen GW- } \\
\text { Neubildung }\end{array}$} & \multirow{3}{*}{$\begin{array}{l}\text { Mischungs- } \\
\text { verhältnisse } \\
\text { Zonen GW- } \\
\text { Neubildung }\end{array}$} & \multirow{2}{*}{$\begin{array}{l}\text { Verweilzeit } \\
\text { Mischungs- } \\
\text { verhältnisse }\end{array}$} \\
\hline & & & & & \\
\hline & & & & & Zonen GW- \\
\hline & & & & Verweilzeit & Neubildung \\
\hline & & & & $\begin{array}{l}\text { Anthropogene } \\
\text { Beeinflussung }\end{array}$ & $\begin{array}{l}\text { Anthropogene } \\
\text { Beeinflussung }\end{array}$ \\
\hline & & & & hydraulische & \\
\hline & & & & Fließwege & $\begin{array}{l}\text { Attenuations- } \\
\text { potential }\end{array}$ \\
\hline
\end{tabular}

Nicht konservativ: Sorption, biol. Abbau, chem. Reaktion, Verflüchtigung bei radioaktiven Isotopen: Zerfall

Die im Kap. 2 zitierte Literatur zeigt, dass der Nutzung natürlich vorkommender Stoffe, wie gelösten Hauptionen oder Metallen der Seltenen Erden, sind durch anthropogene Quellen Grenzen gesetzt sind. Auch anthropogen gebildete Umwelttracer können aus verschiedenen Quellen stammen. Dadurch ist ihr Vorkommen nicht immer spezifisch für einen Eintrags- 
modus, wie beispielsweise die FCKW zeigen, die sowohl aus der Atmosphäre als auch aus Altablagerungen stammen können. Dieses gilt teilweise auch für organische Spurenstoffe, da beispielsweise Medikamente ebenso über Altablagerungen wie auch über das Kanalsystem eingetragen werden können. Viele organische Stoffe lassen jedoch Schlüsse auf einen spezifischen Eintragsmodus oder auch einen Eintragszeitraum zu.

Im Gegensatz zu Umwelttracern und künstlichen Markierungsstoffen ist die Eintragsfunktion organischer Spurenstoffe in der Regel unbekannt, jedoch für die Beantwortung grundlegender Fragestellungen, wie die Abgrenzung von Einzugsgebieten, die Bestimmung von Grundwasserfließrichtungen oder die Überprüfung von Vulnerabilitätsbewertungen auf konzeptioneller Modellebene auch nicht notwendig. Daher können Untersuchungen organischer Spurenstoffe bereits bei geringem Kenntnisstand die Aufstellung konzeptioneller Modelle unterstützen beziehungsweise zu deren Validierung genutzt werden.

Etablierte hydrochemische Methoden setzen in Karstgebieten zum großen Teil nur an der Quelle als integriertes Signal eines Einzugsgebiets an. Organische Spurenstoffe sind hingegen auch in flächiger Verteilung zur Charakterisierung wichtiger Systemeigenschaften nutzbar.

Eine der Hauptzielsetzungen etablierter hydrochemischer Methoden ist die Identifizierung und Quantifizierung von Wasserkomponenten unterschiedlichen Alters oder unterschiedlicher geologischer Herkunft. Zur Abgrenzung von Einzugsgebieten tragen etablierte hydrochemische Methoden durch die Abschätzung von Volumina und darauf aufbauender Abschätzung der Flächengröße bei. Die konkrete Lokalisierung von Einzugsgebietsgrenzen, die Ableitung von Grundwasserfließrichtungen und die Identifizierung von Punktquellen erfordert jedoch zusätzliche Informationen aus der Fläche, die mit an der Quelle ansetzenden Analysen von Isotopenverhältnissen oder Umwelttracern nicht verfügbar sind. Diese Informationen kann die räumliche Verteilung organischer Spurenstoffe liefern.

Die Anteile unterschiedlicher Wasserkomponenten werden zumeist durch Methodenkombinationen identifiziert. Dadurch kommt eine Palette unterschiedlicher Verfahren zum Einsatz, deren Auswertung in vielen Fällen zumindest vereinfachende mathematische Modelle erfordert. Die Untersuchung organischer Spurenstoffe kann notwendige Informationen zur Entwicklung einer konzeptionellen Modellvorstellung auch ohne Anwendung mathematischer Modelle liefern. 
Neben FCKW und vielen der weit verbreiteten monoaromatischen und halogenierten Kohlenwasserstoffen existiert eine große Auswahl nichtflüchtiger organischer Substanzen, bei deren Nutzung ein Austausch mit der ungesättigten Zone auf der Fließstrecke vernachlässigt werden kann.

\subsection{Offene Fragen und Perspektiven der Nutzung organischer Spurenstoffe zur Charakterisierung komplexer Grundwasserleiter}

Der Vorteil der Anwendbarkeit organischer Spurenstoffe ohne Kenntnis der Eintragsfunktion wird zum Nachteil hinsichtlich einer erweiterten Nutzung, beispielsweise für die Anwendung mathematischer Modelle, da die Grundlage zur Erstellung genauer Massenbilanzen fehlt. Diesem Defizit kann in einer ersten Annäherung durch Abschätzung der potentiell eingesetzten Mengen oder durch die Bildung von Verhältnissen der Detektionshäufigkeiten oder Konzentrationen in unterschiedlichen Mengen eingesetzter Stoffe begegnet werden, wie am Beispiel einiger Arzneimittel in Kap. 3 oder Pflanzenschutzmittel in Kap. 5 gezeigt wurde.

Darüber hinaus bietet sich in Gebieten mit identifizierten Eintragsquellen die Möglichkeit, eine Eintragsfunktion zu rekonstruieren und ins Verhältnis zur Konzentration in einer Beobachtungsmessstelle oder zu einer Quelle zu setzen. Hillebrand et al. (2014) nutzen Atrazinund Desethylatrazinkonzentrationen zur Abtrennung des Basisabflusses und zur Erstellung von Massenbilanzen auf Basis einer mittels Flächengröße und üblicher Dosierung geschätzten Einsatzmenge. Auf Grundlage üblicher Verbrauchsmengen schätzen Hillebrand et al. (2012) die Koffeinkonzentration im Abwasser ab und können auf diese Weise den Abwasseranteil einer Karstquelle näherungsweise quantifizieren.

Im Untersuchungsgebiet der Waldecker Scholle könnte ein zeitlich hoch aufgelöstes Monitoring organischer Spurenstoffe im Bereich einer Bachversickerung in Kombination mit Abflussmessungen im Bachlauf ansetzen. Durch ein Parallelmonitoring in der Quelle ließe sich die Basis für Massenbilanzen schaffen, durch die der Beitrag der Bachversickerung am Gesamtquellabfluss bestimmt werden könnte.

Durch künstliche Markierungsversuche und stabile Isotope der Wasseratome werden bevorzugt sehr kurze Verweilzeiten von Stunden bis Wochen oder durch Umwelttracer lange Verweilzeiten von mehreren Jahren bis hin zu Jahrtausenden abgedeckt. Das Stoffspektrum organischer Spurenstoffe umfasst sowohl Stoffe, die im Grundwasser eher konservativ trans- 
portiert werden als auch Stoffe, die einer Attenuation unterliegen. Außerdem sind neben aktuell eingesetzten auch zwischenzeitlich außer Verkehr genommene Stoffe detektierbar. Die Analyse einer entsprechenden Parameterliste hat daher das Potential, die Lücke von Zeiträumen einiger Monaten bis einigen Jahren zu schließen. Diese Zeiträume sind für den Grundwasserschutz bedeutsam, um beispielsweise kurz- bis mittelfristige Auswirkungen von Schadensereignissen prognostizieren zu können. Das Beispiel Atrazin zeigt, dass auch Verweilzeiten von mehr als 20 Jahren erfasst werden können.

Die Nutzung organischer Spurenstoffe bietet vielseitige Implementierungsmöglichkeiten auf verschiedenen Untersuchungsstufen. Bereits in einem frühen Untersuchungsstadium können aus dem Stoffspektrum Rückschlüsse auf die Dynamik des Systems gezogen werden. In detaillierteren Untersuchungen lassen sich durch räumliche oder zeitliche Verteilungsmuster zusätzliche Informationen gewinnen. Die große Anzahl von aktuellen Veröffentlichungen beziehungsweise von Veröffentlichungen aus der jüngeren Vergangenheit zeigt, dass organische Spurenstoffe in zahlreichen Grundwassersystemen vorkommen. Damit ist ihre Nutzung zur Charakterisierung der Eigenschaften dieser Systeme eine zukunftsweisende Methode.

Über die jeweiligen Transporteigenschaften der Stoffe liegen in vielen Fällen nur wenige Informationen vor. Für den Einsatz mathematischer Modelle müsste diese Lücke geschlossen werden. Erste Ansätze dazu finden sich bei Scheytt et al. (2005), Banzhaf et al. (2012), Barbieri et al. 2012 und auch Schaffer et al. (2012) in Form von Batch- und Säulenversuchen ausgewählter Arzneimittel. 


\subsection{Literatur}

Åberg, G.: The use of natural strontium isotopes as tracers in environmental studies. Water Air Soil Poll. 79, 309-322 (1994)

Banzhaf, S., Nödler, K., Licha, T., Krein, A., Scheytt, T.: Redox-sensitivity and mobility of selected pharmaceutical compounds in a low flow column experiment. Sci. Total Environ. 438, 113-121 (2012)

Barbieri, M., Boschetti, T., Petitta, M., Tallini, M.: Stable isotope $\left({ }^{2} \mathrm{H},{ }^{18} \mathrm{O}\right.$ and $\left.{ }^{87} \mathrm{Sr} /{ }^{86} \mathrm{Sr}\right)$ and hydrochemistry monitoring for groundwater hydrodynamics analysis in a karst aquifer (Gran Sasso, Central Italy). Appl. Geochem. 20, 2063-2081 (2005)

Barbieri, M., Licha, T., Nödler, K., Carrera, J., Ayora, C., Sanchez-Vila, X.: Fate of $\beta$-blockers in aquifer material under nitrate reducing conditions: batch experiments. Chemosphere 89, 1272-1277 (2012)

Cook, P.G., Solomon, D.K.: Recent advances in dating young groundwater: chlorofluorocarbons, ${ }^{3} \mathrm{H}^{3} \mathrm{He}$ and ${ }^{85} \mathrm{Kr}$. J. Hydrol. 191, 245-265 (1997)

Field, M.S., Pinsky, P.F.: A two-region nonequilibrium model for solute transport in solution conduits in karstic aquifers. Journal of Contaminant Hydrology 44, 329-351 (2000)

Furtak H., Langguth, H.R.: Zur hydrochemischen Kennzeichnung von Grundwässern und Grundwassertypen mittels Kennzahlen. Mem. IAH-Congress 1965 VII, 86-96 (1967)

Gat, J.R.: Isotope Hydrology: A Study of the Water Cycle. 200 S.; Imperial College Press, London (2010)

Geyer, T., Birk, S., Licha, T., Liedl, R., Sauter, M.: Multitracer test approach to characterize reactive transport in karst aquifers. Ground Water 45, 36-45 (2007)

Goldscheider, N.: Tracer tests in karst hydrogeology and speleology. International Journal of Speleology, 37, 27-40 (2008)

Hillebrand, O., Nödler, K., Geyer, T., Licha, T.: Investigating the dynamics of two herbicides at a karst spring in Germany: Consequences for sustainable raw water management. Sci. Total Environ. 482-483, 193-200 (2014) 
Hillebrand, O., Nödler, K., Licha, T., Sauter, M., Geyer, T.: Caffeine as an indicator for the quantification of untreated wastewater in karst systems. Water Res. 46, 395-402 (2012)

Hölting. B., Matthess, G.: Ein Sporentriftversuch im Zechstein von Korbach/ Hessen. Notizbl. HlfB 91, 237-255 (1963)

Johannesson, K.H., Stetzenbach, K.J., Hodge, V.F., Kreamer, D.K., Zhou, X.: Delineation of ground-water flow systems in the Southern Great Basin using aqueous rare earth element distributions. Ground Water 35, 805-819 (1997)

Käss, W., Behrens, H., Matthess, G.: Geohydrologische Markierungstechnik. 557 S.; Borntraeger, Berlin Stuttgart (2004)

Katz, B.G., Chelette, A.R., Pratt, T.R.: Use of chemical and isotopic tracers to assess nitrate contamination and groundwater age, Woodville Karst Plain, USA. J. Hydrol. 289, 36-61 (2004)

Kendall, C., McDonnell, J.J. (Eds.): Isotope Tracers in Catchment Hydrology. 839 S.; Elsevier Science B.V., Amsterdam (1998)

Long, A.J., Sawyer, F., Puntnam, L.D.: Environmental tracers as indicators of karst conduits in groundwater in South Dakota, USA. Hydrogeol. J. 16, 263-280 (2008)

Maloszewski, P., Zuber, A.: Determining the turnover time of groundwater systems with the aid of environmental tracers. J. Hydrol. 57, 207-231 (1982)

Pinault, J.-L., Plagnes, V., Aquilina, L.: Inverse Modeling of the hydrological and the hydrochemical behavior of hydrosystems: Characterization of karst system functioning. Water Res. Res. 37, 2191-2204 (2001)

Piper, A.M.: A graphic procedure in the geochemical interpretation of water analysis.- Trans. Am. Geophys. Union 25, 914-928 (1944)

Schaffer, M., Boxberger, N., Börnick, H., Licha, T., Worch, E.: Sorption influenced transport of ionizable pharmaceuticals onto a natural sandy aquifer sediment at different $\mathrm{pH}$. Chemosphere 87, 513-520 (2012) 
Scheytt, T., Mersmann, P., Lindstädt, R., Heberer, T.: 1-Octanol/Water Partition Coefficients of 5 Pharmaceuticals from Human Medical Care: Carbamazepine, Clofibric Acid, Diclofenac, Ibuprofen, and Propyphenazone. Water Air Soil Poll. 165, 3-11 (2005)

Sidle, W.C.: Environmental isotopes for resolution of hydrology problems. Environ. Monit. Assess. 52, 389-410 (1998)

Solomon, D.K., Cook, P.G., Sanford, W.E.: Dissolved gases in subsurface hydrology. In: Kendall, C., McDonnell, J.J. (Eds.): Isotope tracers in catchment hydrology. 839 S.; Elsevier Science B.V., Amsterdam (1998)

Sültenfuß, J., Massmann, G.: Datierung mit der ${ }^{3} \mathrm{He}$-Tritium-Methode am Beispiel der Uferfiltration im Oderbruch. Grundwasser 9, 221-234 (2004)

Tallini, M., Falcone, R.A., Carucci, V., Falgiani, A., Parisse, B., Petitta, M.: Isotope hydrology and geochemical modeling: new insights into the recharge processes and water-rock interactions of a fissured carbonate aquifer (Gran Sasso, central Italy). Environ. Earth Sci. DOI 10.1007/s12665-014-3364-9 (2014) 


\section{Anhang}

Schriften und Konferenzbeiträge im Zusammenhang mit dieser Dissertation:

\section{Veröffentlichungen in Fachzeitschriften}

Reh R., Licha T., Geyer T., Nödler, K., Sauter M.: Occurrence and spatial distribution of organic micro-pollutants in a complex hydrogeological karst system during low flow and high flow periods, results of a two-year study. Sci. Total Environ. 443, 438-445 (2013)

Reh, R., Licha, T., Nödler, K., Geyer, T., Sauter, M.: Evaluation and application of organic micro-pollutants (OMPs) as indicators in karst system characterization. Environ. Sci. Pollut. Res. DOI 10.1007/s11356-014-3676-z (2014)

Reh, R., Hillebrand, O., Geyer, T., Nödler, K., Licha, T., Sauter, M.: Charakterisierung zweier Karstsysteme mit Hilfe organischer Spurenstoffe. Grundwasser 19, 251-262 (2014)

\section{Konferenzbeiträge}

Reh, R., Licha, T, Geyer, T., Nödler, K., Sauter, M.: Organic compounds as indicators for transport in an urban characterized complex karst system. Geophysical Research Abstracts 14, EGU 2012-9440-1, EGU General Assembly 2012, Vienna, Austria (2012)

Reh, R., Licha, T, Geyer, T., Nödler, K., Sauter, M. : Organische Spurenstoffe als Transport-Indikatoren in einem urban geprägten komplexen Karstsystem. Schriftenreihe der Deutschen Gesellschaft für Geowissenschaften 78, S. 49, Dresden, Deutschland (2012)

Reh, R., Licha, T, Geyer, T., Nödler, K., Sauter, M.: Organic micro-pollutants in a karst system during low and high flow periods, results of a two-year study. Geophysical Research Abstracts 15, EGU 2013-8753, EGU General Assembly 2013, Vienna, Austria (2013) 


\section{Sonstige}

Licha T, Nödler K, Geyer T, Reh R, Hillebrand O, 2011. Spurenorganika im Wasserkreislauf neue Perspektiven in der Hydrogeologie. HLUG-Seminar Altlasten und Schadensfälle (2011), Wetzlar, Deutschland (2011)

Reh, R., Licha, T, Geyer, T., Nödler, K., Sauter, M, Ezzel, B., Weidemann, M.: Hydrodynamik und Stofftransport in einem urban geprägten Karstgrundwassersystem - Schutz der Trinkwassergewinnungsanlagen in Korbach. Projektbericht im Rahmen des Kooperationsvertrags der Universität Göttingen mit dem HLUG und der EWF GmbH, 1. Überarbeitung (2012)

Reh, R., Licha, T, Geyer, T., Nödler, K., Sauter, M., Schloesser-Kluger, I., Jaeger-Wunderer, M.: Hydrodynamik und Stofftransport in einem urban geprägten Karstgrundwassersystem. Jahresbericht HLUG 2013, 43-50 (2013)

Reh, R., Licha, T, Geyer, T., Nödler, K., Sauter, M.: Organische Spurenstoffe, Nutzung der Indikatorfunktion in einem komplexen Grundwassersystem. HLUG-Seminar Altlasten und Schadenfälle 2013, Limburg, Deutschland (2013

\section{Master- und Bachelorarbeiten}

Ezzel, B.: Characterization of a karst aquifer system with hydraulic tests: Korbach, Germany. M.Sc., Georg-August-Universität Göttingen, GZG (unveröffentlicht) (2011)

Meyerink, Tristan: Hydrochemische und isotopenhydrologische Untersuchung von Grundwasserquellen im Raum Korbach und Göttingen. B.Sc. Georg-August-Universität Göttingen, GZG (unveröffentlicht) (2012)

Weidemann, M. : Development of a water balance for the catchment areas of the rivers Itter, Twiste and Werbe (Ldkrs. Waldeck Frankenberg), M.Sc., Georg-August-Universität Göttingen, GZG (unveröffentlicht) (2011) 


\section{Lebenslauf}

\section{Persönliche Daten:}

Name: $\quad$ Roland Reh

Geburtsdatum: $\quad 30.03 .1969$

Geburtsort: $\quad$ Göttingen, Deutschland

Nationaltät: deutsch

\section{Studium/ Ausbildung:}

1990 - 1997: Studium der Geologie an der Georg-August-Universität Göttingen, Abschluss: Diplom

Thema der Diplomarbeit:

Geologische Kartierung zwischen Petersdorf und Krimderode (TK 25: 4430 Nordhausen-Nord und TK 25: 4431 Stolberg am Harz) und Faziesanalyse des Zechsteins in der Bohrung Baldenhain 4326/76 (TK 25; 5639 Kayna) in Osthüringen

2008: $\quad$ Öffentliche Bestellung als Sachverständiger für Gefährdungsabschätzung für den Wirkungspfad Boden - Gewässer (Bodenschutz und Altlasten, Sachgebiet 2), Zuständigkeit: IHK Kassel

\section{Beruflicher Werdegang:}

1997 - 1999 Sachbearbeitung und kommissarische Sachgebietsleitung bei der Stadt Kassel, Untere Wasserbehörde, Altlasten u. Schadensfälle

1999 - 1/2009 Tätigkeit als Altlasten-Sachverständiger bei: Das Baugrund Institut Dipl.-Ing. Knierim $\mathrm{GmbH}$

seit 2/2009 Geschäfsführer Geonik GmbH, Kassel 


\section{Weitere Veröffentlichungen/ Konferenzbeiträge:}

Paul, J., Quast, A., Ahlborn, F., Plache, M., Reh, R.: Geologie des Gipskarstgebiets zwsichen Nordhausen und Stempeda (Zechstein, südlicher Harzrand). Geowiss. Mitt. von Thüringen 6, 57-81 (1998)

Marczinek, S., Breiter, R., Peiffer, S., Reh, R., Woisnitza, M.: Aerobic Degradation of 1,2-DCP and 1,2-DCA in a contaminated groundwater plume at a former Industrial Site. 2nd European Conference on Natural Attenuation, Soil and Groundwater Risk Management, Frankfurt am Main (2005) 